\title{
Helical Carbenium Ion: A Versatile Organic Photoredox Catalyst in Red- Light-Mediated Reactions
}

\author{
Liangyong Mei, José M. Veleta, and Thomas L. Gianetti* \\ Department of Chemistry and Biochemistry, University of Arizona, Tucson, Arizona 85721, United States
}

\section{Supporting Information}

\begin{abstract}
Red light has the advantages of low energy, less health risk and high penetration depth through various media. Herein, a helical carbenium ion ( $N, N$ '-di- $n$-propyl-1,13-dimethoxyquinacridinium ( $n$ Pr-DMQA + ) tetrafluoroborate) has been used as an organic photoredox catalyst for photoreductions and photooxidations in the presence of red light $\left(\lambda_{\max }=640 \mathrm{~nm}\right)$. It has catalyzed red-lightmediated dual transition-metal/photoredox-catalyzed $\mathrm{C}-\mathrm{H}$ arylation and intermolecular atom transfer radical addition through oxidative quenching, affording products in 57-93\% yields. Moreover, its potential in photooxidation catalysis has also been demonstrated by successful applications in red-light-induced aerobic oxidative hydroxylation of arylboronic acids and benzylic $\mathrm{C}\left(\mathrm{sp}_{3}\right)$ - $\mathrm{H}$ oxygenation through reductive quenching, delivering products in up to $92 \%$ yield. Thus, a versatile organic photoredox catalyst (helical carbenium ion) for red-light-mediated photoredox reactions has been developed.
\end{abstract}

Over the past decade, photoredox catalysis has taken the world of synthetic chemistry by a storm.1 Iridium and ruthenium polypyridyl complexes such as $\mathbf{1} \mathbf{a}_{2}$ and $\mathbf{1} \mathbf{b}_{3}$ are among the most powerful photoredox catalysts (PCs) due to their unique properties such as stable and long-lived excited states, large redox windows, and as effective excited state oxidants and reductants (Figure 1). However, given the intrinsic disadvantages of transition-metal catalysts such as high cost, low sustainability and potential toxicity, researchers have been looking for inexpensive and environmental-friendly alternatives. In recent years, organic molecules such as acridiniums (e.g. 9-mesityl-10methylacridinium 1c) or xanthene dyes (e.g. Eosin Y 1d) have proven to be efficient alternatives (Figure 1).4 Despite the general merits of organic PCs, there are disadvantages to overcome. For example, the most widely used catalyst in this category 1c, first introduced by Fukuzumis and expanded by Nicewicz,4,6 is mainly used for reductive quenching. Similarly, the narrow redox window, $\mathrm{pH}$ dependence, and susceptibility to bleaching have also rendered 1d less effective.7 Therefore, developing more versatile organic PCs is still highly desirable.

Figure 1. Commonly Used Photoredox Catalysts

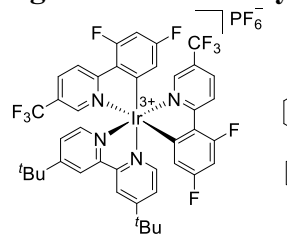

1 a

$\left[\mathrm{Ir}\left(\mathrm{dF}-\mathrm{CF}_{3}-\mathrm{ppy}\right)_{2}(\mathrm{dtbpy})\right]\left(\mathrm{PF}_{6}\right)$ $\lambda_{\text {max }}=380 \mathrm{~nm}$ (Violet)

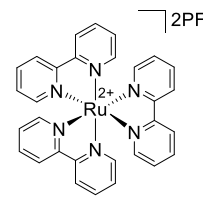

1b

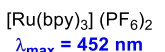

$\lambda_{\max }=452 \mathrm{~nm}$
(Blue)

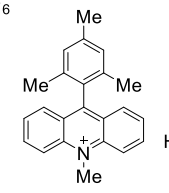

$1 \mathrm{c}$ Mes-Acr-Me ${ }^{+}$ $\lambda_{\max }=430 \mathrm{~nm}$

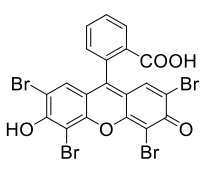

10

Eosin $Y$ $\lambda_{\max }=539 \mathrm{~nm}$ (Green)
Despite the impressive progress in photoredox catalysis to date, most light-induced reactions are accomplished under high-energy blue, green or white light.1,4 Besides the health hazards (photooxidative damage to retina), 8 blue and green light are also more prone to induce undesired mixtures due to the possible photon absorptions by reactants or products. 9 In contrast, red light (600$700 \mathrm{~nm}$ ) has advantages such as low energy, fewer side reactions, less health risk and is naturally abundant from sunlight. More importantly, it penetrates turbid media, and great success have been made in its biology-related applications.10 Yet reports on red-light-mediated synthetic methodologies still remain scarce, and only handful examples can be found (Scheme 1a). Ferroud and coworkers introduced a red-light-induced methylene blue (2a)-catalyzed aerobic photooxidation and photocyanation of hydrazines (Scheme 1a, I).11 Though more applications of 2a in photocatalysis have been reported, they all employ blue, green or white light.4,12 Similarly, PCs $\mathbf{2 b - 2 d}$ have also achieved some successes in red-light-mediated methodologies, while no further applications was conducted (Scheme 1a, II-IV).13 Notably, Rovis and Campos have recently introduced an elegant work on nearinfrared light-induced transformations in the absence of a PC, or in the presence of $\mathbf{1 b}, \mathbf{1 d}$, or Rose Bengal via triplet fusion upconversion, 14 followed by the development of a series of Os(II)-based PCs for infrared photoredox catalysis.15 These important examples all suggest that applying red light in photoredox catalysis is of great significance.

The helical carbenium ion - dimethoxyquinacridinium (DMQA+), has been well-studied regarding its photophysical,16 electrochemical properties, $16 \mathrm{~b}, 17$ and post-functionalization $16 \mathrm{~b}, 18$ since its discovery by Laursen and coworkers.16a Applications of DMQA + have been primarily reserved to the biological arena.19 In 2005, the Lacour group revealed the first and only example of photocatalysis using DMQA+, which demonstrated the aerobic photooxidation of benzylamine in the presence of $N, N^{\prime}$-di- $n$ propyl-1,13-dimethoxyquinacridinium (nPr-DMQA+) tetrafluoroborate (3) under photoirradiation (600 W lamp) to afford benzylimine in $15 \%$ NMR yield at $70{ }^{\circ} \mathrm{C} .20$ Based on the reported extraordinary photophysical and electrochemical properties of 3, as well as Lacour's results on photocatalysis, we speculated that 3 could serve as a versatile organic PC. Herein, we report the photoredox properties of $\left[{ }_{n} \mathrm{Pr}-\mathrm{DMQA}_{+}\right]\left[\mathrm{BF}_{4}-\right]$ (3), and its reactivity in a wide range of red-light-mediated reactions including reductive and oxidative quenching (Scheme 1b). 
Scheme 1. (a) Previous Work on Red-Light-Mediated Methodologies and (b) a Versatile Organic PC in This Work

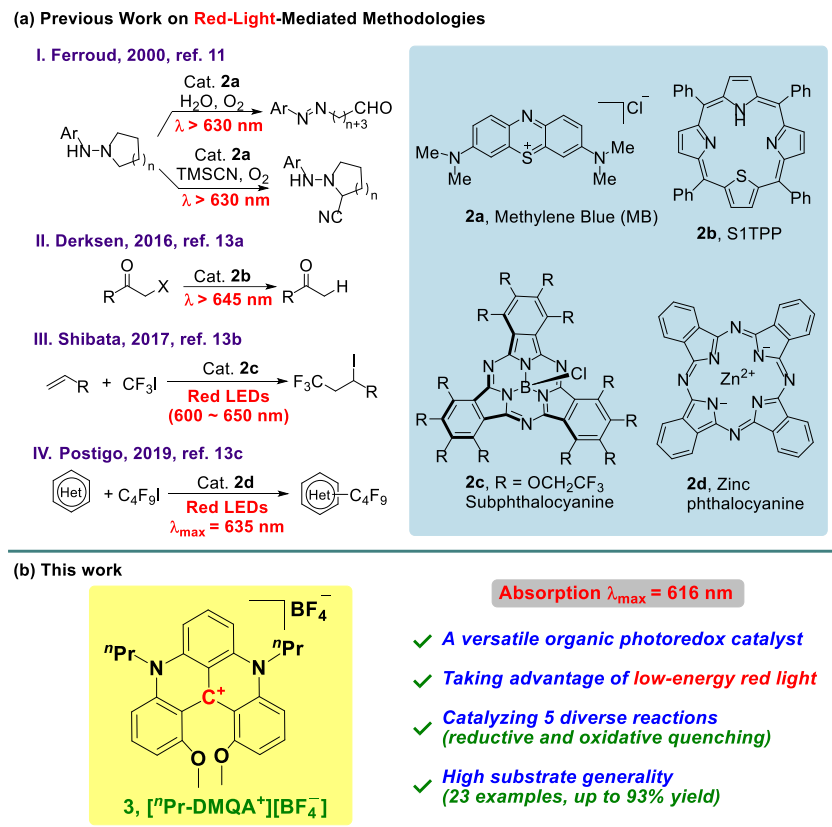

Before testing the photocatalytic reactivity of $\mathbf{3}$, we first calculated the excitation energy $\left(\mathrm{E}_{0,0}\right)$, excited state oxidation $\left(\mathrm{E}_{1 / 2}\right.$ $(\mathrm{C} \cdot++/ \mathrm{C}+*))$ and reduction potentials $\left(\mathrm{E}_{1 / 2}(\mathrm{C}+* / \mathrm{C} \cdot)\right)$ based on the reported absorption and emission spectra16b and cyclic voltammetry data17 (Table S1, see details in Supporting Information).4a To our delight, $\mathbf{3}$ possesses moderate ground state oxidation and reduction potentials $\mathrm{E}_{1 / 2}\left(\mathrm{C} \cdot++/ \mathrm{C}_{+}\right)=+1.32 \mathrm{~V}$ and $\mathrm{E}_{1 / 2}(\mathrm{C}+/ \mathrm{C} \cdot)=-0.78 \mathrm{~V}$ vs. the saturated calomel electrode (SCE), as well as moderate excited state oxidation and reduction potentials $\mathrm{E}_{1 / 2}(\mathrm{C} \cdot++/ \mathrm{C}+*)=-0.61 \mathrm{~V}$ and $\mathrm{E}_{1 / 2}(\mathrm{C}+* / \mathrm{C} \cdot)=+1.15 \mathrm{~V}$ (Table S1 and Scheme 3). These features render 3 a mild reductant and oxidant whether a reductive or oxidative quenching is involved during photocatalysis. Moreover, the excited state lifetime $(\tau=5.5 \mathrm{~ns}) 16 \mathrm{~b}$ is comparable to those of other commonly used organic PCs (2 $20 \mathrm{~ns}$ in general). 4 In particular, the peak absorption $\left(\lambda_{\max }\right)$ is 616 $\mathrm{nm}, 16 \mathrm{~b}$ which makes our goal of taking advantage of low-energy red light feasible.

Initial reactivity studies began with a red-light-mediated dual $\mathrm{Pd} / n \mathrm{Pr}-\mathrm{DMQA}+$-catalyzed $\mathrm{C}\left(\mathrm{sp}_{2}\right)-\mathrm{H}$ arylation, which was first reported by the Sanford group using Ru(bpy) $32+$ in the presence of $26 \mathrm{~W}$ compact fluorescent light (CFL).21 In the reported reaction, $\mathrm{Ru}(\text { bpy })_{32+}\left(\mathrm{E}_{1 / 2}(\mathrm{Ru}(\mathrm{III}) / \mathrm{Ru}(\mathrm{II} *))=-0.81 \mathrm{~V}\right.$ vs. SCE) acted as a photoreductant by reducing $\mathrm{ArN}_{2}+$ to aryl radical $\left(\mathrm{PhN}_{2} / \mathrm{Ph} \cdot=-\right.$ $0.10 \mathrm{~V}$ vs. SCE) 22 through oxidative quenching, followed by oxidation of $\mathrm{Pd}(\mathrm{III})$ to $\mathrm{Pd}(\mathrm{IV})$ to regenerate $\mathrm{Ru}(\mathrm{bpy})_{32+}$ $\left(\mathrm{E}_{1 / 2}(\mathrm{Ru}(\mathrm{III}) / \mathrm{Ru}(\mathrm{II}))=+1.29 \mathrm{~V}\right.$ vs. SCE $)$. Given $\mathrm{E}_{1 / 2}(\mathrm{C} \cdot++/ \mathrm{C}+*)=-$ $0.61 \mathrm{~V}$ and $\mathrm{E}_{1 / 2}(\mathrm{C} \cdot++/ \mathrm{C}+)=+1.32 \mathrm{~V}$ for 3 , this reaction was deemed suitable to display its photoreduction ability. To our delight, the reaction between 1-([1,1'-biphenyl]-2-yl)pyrrolidin-2one $4 \mathbf{a}$ and benzenediazonium tetrafluoroborate $5 \mathbf{a}$ proceeded smoothly in the presence of $\mathrm{Pd}(\mathrm{OAc})_{2}$ and $\mathbf{3}$ under red LED $\left(\lambda_{\max }\right.$ $=640 \mathrm{~nm}$ ), affording the desired product 6a in 95\% NMR yield after 4 hours (Table S2). Consistent with Sanford's work,21 in the absence of $\mathbf{3}$, red light, or $\mathrm{Pd}(\mathrm{OAc}) 2$, significantly lower yields ( $\leq$ $25 \%$ ) of 6 a was observed (Table S2).

With satisfying results in hand, we sought to expand the substrate scope of this red-light-mediated dual $\mathrm{Pd} / n \mathrm{Pr}-\mathrm{DMQA}+-$ catalyzed $\mathrm{C}\left(\mathrm{sp}_{2}\right)-\mathrm{H}$ arylation (Table 1). Using 4a as model substrate, electron-neutral, electron-deficient and electron-rich aryldiazonium salts $\mathbf{5 a - 5 c}$ were tested. Reactions proceeded smoothly, delivering the desired products 6a-6c in $86-93 \%$ yields (Table 1, entry 1). Substrate $\mathbf{4 b}$ containing pyridine as the directing group (DG) was well-tolerated as well, furnishing the corresponding products $\mathbf{6 d - 6 f}$ in $60-73 \%$ yields by reacting with $\mathbf{5 a - 5 c}$ (entry 2 ). In addition, the desired $\mathrm{C}-\mathrm{H}$ arylated product $\mathbf{6 g}$ could also be obtained in $57 \%$ yield when $\mathbf{4 c}$ with pyrimidine as the directing group reacted with 5a (entry 3 ). Lastly, oxime $\mathbf{6 h}$ was isolated in $64 \%$ yield when $\mathbf{4 d}$ was reacted with $\mathbf{5 a}$ (entry 4 ). Notably, all the products $\mathbf{6}$ were obtained in relatively higher yields compared to the previous work except for $\mathbf{6 d} .21$ Furthermore, control experiments in the absence of $\mathbf{3}$, red light or $\mathrm{Pd}(\mathrm{OAc})_{2}$ resulted in much lower yields for all substrates (Table $\mathrm{S} 3$ ). The successful application of $\mathbf{3}$ in this red-light-mediated C$\mathrm{H}$ arylation demonstrates that it is capable of engaging in oxidative quenching during photocatalysis.

Table 1. Red-Light-Mediated Dual Pd/nPr-DMQA+Catalyzed C(sp2)-H Arylationa
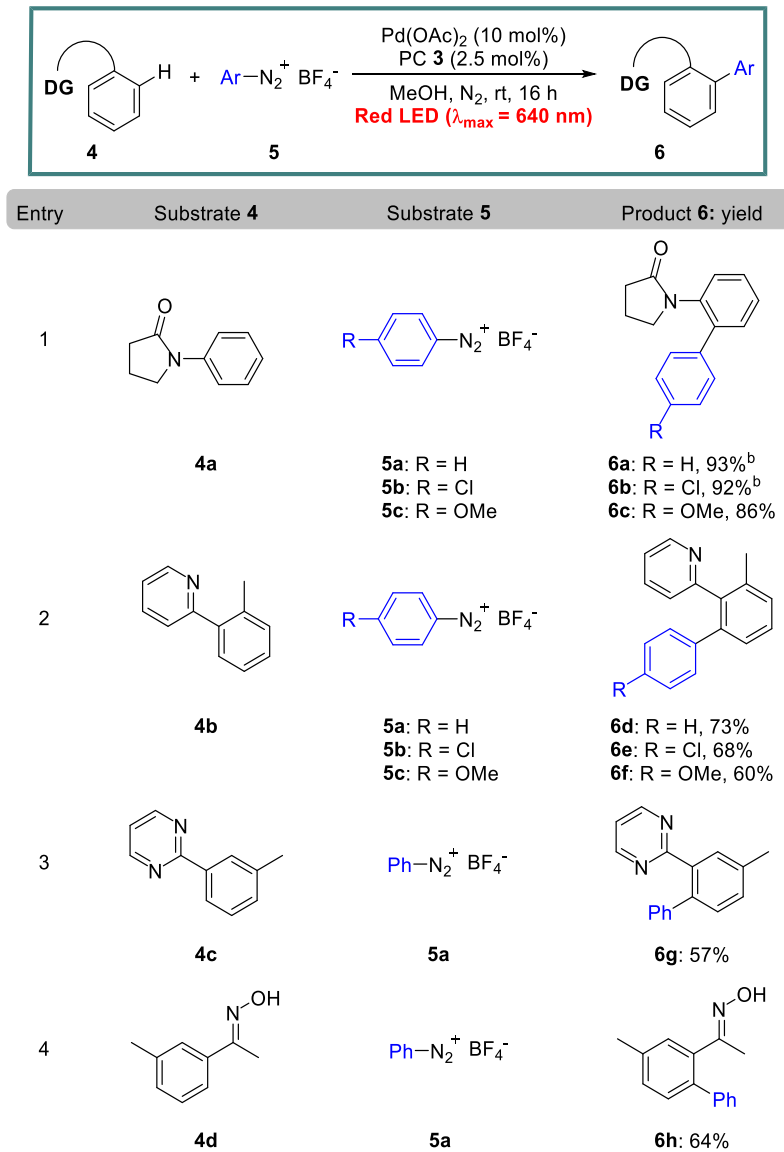

aReactions conducted on $0.2 \mathrm{mmol}$ scale. Isolated yields shown. $\mathrm{bThe}$ reaction was ran for $4 \mathrm{~h}$.

Next, we turned our attention to photo-induced aerobic oxidative hydroxylation of arylboronic acids, which is a welldocumented reaction via reductive quenching under white or blue light.23 The reaction mainly involves the oxidation of ${ }_{i} \operatorname{Pr}_{2} \mathrm{NEt}$ (DIPEA) by an excited state $\mathrm{PC} *$ to form the radical cation ${ }_{i} \operatorname{Pr}_{2} \mathrm{NEt} \cdot+{ }_{(i \operatorname{Pr} 2 \mathrm{NEt}} /{ }_{\mathrm{Pr}} \mathrm{NEt} \cdot+=+0.72 \mathrm{~V}$ vs. SCE$)_{24}$ and the reduction of $\mathrm{O}_{2}$ by the $\mathrm{PC}$ - to generate the superoxide radical anion $\mathrm{O}_{2 \cdot-}\left(\mathrm{O}_{2} / \mathrm{O}_{2 \cdot-}=-0.57 \mathrm{~V}\right.$ vs SCE. $) .24 \mathrm{a}, 25 \mathrm{With} \mathrm{E}_{1 / 2}(\mathrm{C}+* / \mathrm{C} \cdot)$ at $+1.15 \mathrm{~V}$ and $\mathrm{E}_{1 / 2}\left(\mathrm{C}_{+} / \mathrm{C} \cdot\right)$ at $-0.78 \mathrm{~V}, 3$ should be competent to serve as an efficient PC for this reaction. Gratifyingly, phenol 8a was obtained in $87 \%$ NMR and $83 \%$ isolated yield when phenylboronic acid 7a was treated with DIPEA and $\mathbf{3}$ in the presence of air and red light for 24 hours in DMF (Table S4). In 
accordance with reported results, 23 in the absence of PC or red light, little or no conversion was observed (Table S4).

Substrate scope results for oxidative hydroxylation of arylboronic acids are presented in Table 2. A wide range of arylboronic acids 7 with diverse useful functional groups such as halide (7c), nitrile (7d), aldehyde (7e), ester (7f), carboxylic acid $(7 \mathbf{g})$ and nitro (7k) were well-tolerated, giving rise to the desired phenols 8a-81 in moderate to high yields. Additionally, the substitution pattern on the phenyl ring or electronic properties of 7 did not have much influence over the reaction outcome. For example, 8b-8g were isolated in $41-87 \%$ yields when $\mathbf{7 b}-\mathbf{7 g}$ with different electron-donating groups (EDG) or electron-withdrawing groups (EWG) at para position were examined. Substrates $\mathbf{7 h - 7 k}$ with diverse substituents at ortho or meta position also provided the corresponding phenols $\mathbf{8 h - 8 k}$ in $55-73 \%$ yields. 2Naphthylboronic acid 7l was also suitable for this oxidative hydroxylation, providing naphthalen-2-ol $\mathbf{8 1}$ in $65 \%$ yield. This red-light-induced ${ }_{n}$ Pr-DMQA+-catalyzed aerobic oxidative hydroxylation shows that $\mathbf{3}$ is an efficient PC for photocatalysis involving reductive quenching.

Table 2. ${ }_{n}$ Pr-DMQA+-Catalyzed Aerobic Oxidative Hydroxylation of Arylboronic Acids under Red Lighta

\begin{tabular}{|c|c|c|}
\hline \multirow{2}{*}{$\operatorname{ArB}(\mathrm{OH})_{2}$} & $\begin{array}{c}\text { DIPEA } \\
\text { PC } 3 \text { (2 } 2 \text { ol } \%)\end{array}$ & \multirow{2}{*}{$\mathrm{ArOH}$} \\
\hline & DMF, air, rt, 24 h & \\
\hline 7 & $\operatorname{Red} \operatorname{LED}\left(\lambda_{\max }=640 \mathrm{~nm}\right)$ & 8 \\
\hline
\end{tabular}

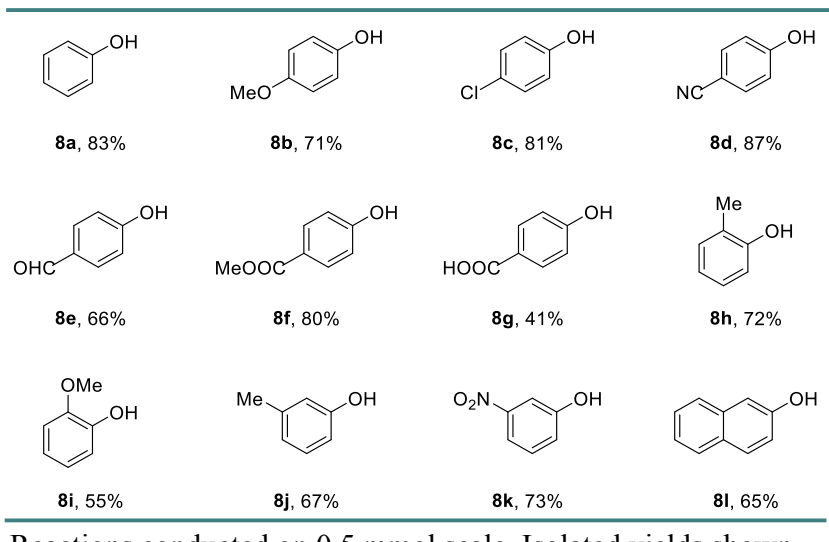

aReactions conducted on $0.5 \mathrm{mmol}$ scale. Isolated yields shown.

To further illustrate the generality of $\mathbf{3}$ as a PC, additional redlight-induced transformations were investigated (Scheme 2). As another classic example of photocatalysis involving in reductive quenching and $\mathrm{O}_{2} \cdot$-, visible-light-mediated aerobic benzylic $\mathrm{C}\left(\mathrm{sp}_{3}\right)-\mathrm{H}$ oxygenation using oxygen as the oxidant was extensively studied.26 When a tertiary amine 9 was treated with 1,8-diazabicyclo[5.4.0] undec-7-ene (DBU) and 3 in the presence of air and red light, amide $\mathbf{1 0}$ was isolated in $92 \%$ yield (Scheme 2a). Atom transfer radical addition (ATRA) of organic halides to olefins serves as an atom-economical approach of simultaneously forming $\mathrm{C}-\mathrm{C}$ and $\mathrm{C}-\mathrm{X}$ bonds.27 As presented in Scheme $2 \mathrm{~b}$, in the presence of $\mathrm{LiBr}$ and $\mathbf{3}$, a red-light-induced reaction between 4 nitrobenzyl bromide $\mathbf{1 1}$ and styrene $\mathbf{1 2}$ was realized, affording the desired adduct $\mathbf{1 3}$ in 59\% yield (see Table S5 and S6 for more optimization and Scheme S1 for mechanism). At last, 3 could also act as a viable alternative for Glorius's dual gold/photoredoxcatalyzed $\mathrm{C}(\mathrm{sp})-\mathrm{H}$ arylation of terminal alkyne $\mathbf{1 4}$ with $\mathbf{5 a}, 28$ providing the desired product $\mathbf{1 5}$ in $62 \%$ yield (Scheme $2 \mathrm{c}$, see more optimization in Table S7). Control experiments in the absence of $\mathbf{3}$, red light, or other reagents such as $\mathrm{DBU}, \mathrm{LiBr}$ or $\mathrm{Au}\left(\mathrm{PPh}_{3}\right) \mathrm{Cl}$ were also performed (Scheme S2). The reduced yields of 10, 13, and 15 further supports the essential roles of 3 and red light for these reactions.

Scheme 2. Additional Examples of Red-Light-Induced ${ }_{n}$ PrDMQA+-Catalyzed Reactionsa
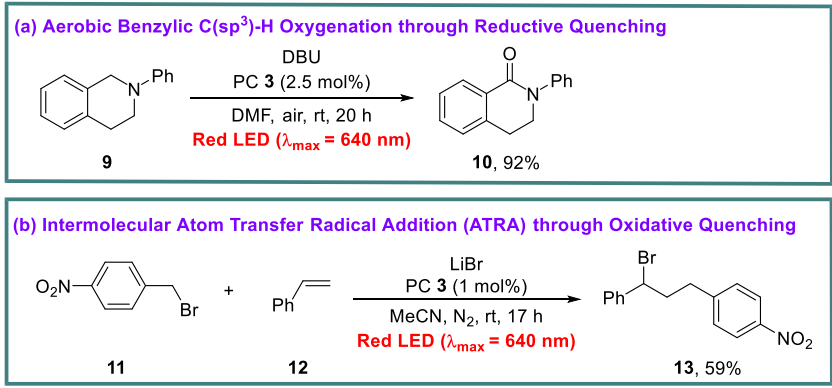

(c) Dual Au/ ${ }^{n} \mathrm{Pr}^{-\mathrm{DMQA}^{+} \text {-Catalyzed C(sp)-H Arylation through Oxidative Quenching }}$

aReactions conducted on $0.2 \mathrm{mmol}$ scale. Isolated yields shown.

Based on previous work,21,23 two plausible catalytic cycles involving in oxidative or reductive quenching are proposed (Scheme 3). Scheme 3-I shows proposed mechanism for dual $\mathrm{Pd} / n \mathrm{Pr}$-DMQA+-catalyzed $\quad \mathrm{C}\left(\mathrm{sp}_{2}\right)-\mathrm{H} \quad$ arylation.21 Firstly, photoexcitation of $\mathbf{3}$ generates ${ }_{n} \operatorname{Pr}-\mathrm{DMQA}+*$, which reduces $\mathbf{5}$ to form an aryl radical and ${ }_{n}$ Pr-DMQA.++ through oxidative quenching. Then, addition of the aryl radical to the $\operatorname{Pd}(\mathrm{II})$ intermediate A (generated by $\mathrm{C}-\mathrm{H}$ activation of substrate 4) affords the $\operatorname{Pd}(\mathrm{III})$ species $\mathbf{B}$, followed by an one-electron oxidation with ${ }_{n} \mathrm{Pr}-\mathrm{DMQA} \cdot++$ to regenerate ${ }_{n} \mathrm{Pr}-\mathrm{DMQA}+$ and form the $\operatorname{Pd}(\mathrm{IV})$ intermediate $\mathrm{C}$. Lastly, reductive elimination of the intermediate $\mathbf{C}$ furnishes the arylated product $\mathbf{6}$. Mechanism for ${ }_{n} \mathrm{Pr}-\mathrm{DMQA}+-$ catalyzed aerobic oxidative hydroxylation is presented in Scheme 3-II.23 ${ }_{i} \operatorname{Pr}_{2} \mathrm{NEt}$ is first oxidized to an ammonium radical cation by the excited state of $\mathbf{3}$, along with formation of a helicene radical $n$ Pr-DMQA through reductive quenching. Then, the helicene radical, which has been previously observed and characterized in situ by Laursen,17 further reacts with oxygen to regenerate 3 and form an $\mathrm{O}_{2} \cdot$. Finally, follow-up oxidative attack onto substrates $\mathbf{7}$ and hydrolysis afford phenols 8 .

Scheme 3. Plausible Catalytic Cycles For (I) C(sp2)-H Arylation, and (II) Aerobic Oxidative Hydroxylation

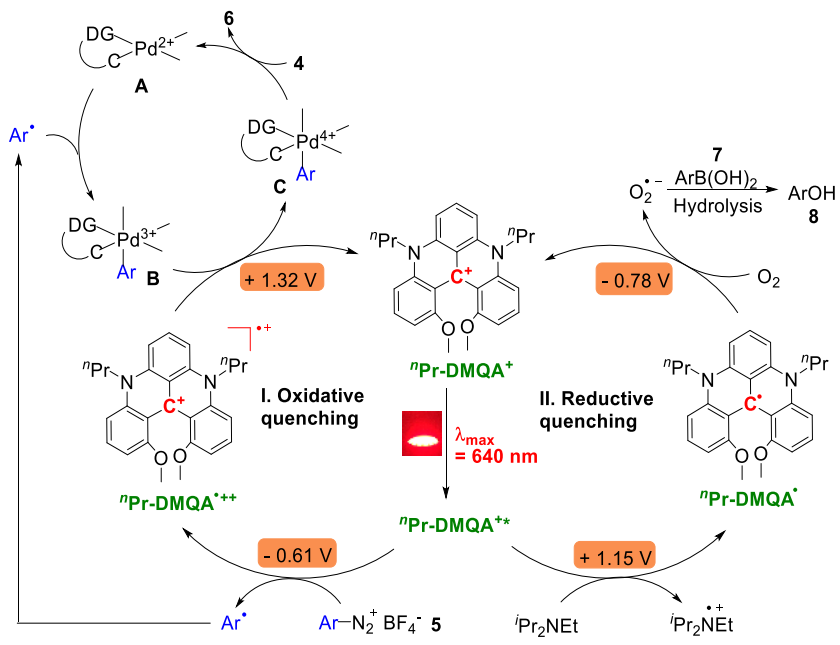


In summary, we have disclosed a helical carbenium ion $-\left[{ }_{n} \mathrm{Pr}-\right.$ DMQA+][BF4-] (3), which catalyzes photoreductions and photooxidations in the presence of low-energy red light. The role of $\mathbf{3}$ as an efficient $\mathrm{PC}$ in oxidative and reductive quenching were evaluated by transition-metal $/ n \operatorname{Pr}-\mathrm{DMQA}+$-catalyzed $\mathrm{C}-\mathrm{H}$ arylations and intermolecular ATRA (oxidative quenching), as well as aerobic oxidative hydroxylation of arylboronic acids and benzylic $\mathrm{C}\left(\mathrm{sp}_{3}\right)-\mathrm{H}$ oxygenation (reductive quenching). Eight diverse substrates were well-tolerated for red-light-mediated dual $\mathrm{Pd} / n \mathrm{Pr}-\mathrm{DMQA}+-$ catalyzed $\mathrm{C}\left(\mathrm{sp}_{2}\right)-\mathrm{H}$ arylation. Moreover, with twelve different arylboronic acids as the substrates, red-lightinduced ${ }_{n} \mathrm{Pr}$-DMQA+-catalyzed aerobic oxidative hydroxylation proceeded smoothly as well. The successful applications of $\mathbf{3}$ in these red-light-mediated reactions have established its role as a versatile organic $\mathrm{PC}$, which can serve as a complementary option for current white, blue or green-light-mediated photocatalysis. Further investigations on the applications of $\mathbf{3}$ towards more challenging photoredox catalysis are in progress.

\section{ASSOCIATED CONTENT}

\section{Supporting Information}

The Supporting Information contains experimental procedures, characterization, and spectral data (PDF)

\section{AUTHOR INFORMATION}

\section{Corresponding Author}

Thomas L. Gianetti - Department of Chemistry and Biochemistry, University of Arizona, Tucson, Arizona 85721, United States; https://orcid.org/0000-0002-3892-3893

Email: tgianetti@arizona.edu.

\section{Authors}

Liangyong Mei - Department of Chemistry and Biochemistry, University of Arizona, Tucson, Arizona 85721, United States; https://orcid.org/0000-0002-4704-4030;

Email: lymei@arizona.edu.

José M. Veleta - Department of Chemistry and Biochemistry, University of Arizona, Tucson, Arizona 85721, United States; https://orcid.org/0000-0002-5272-2131

Email: jmveleta@arizona.edu.

Notes

The authors declare no competing financial interest.

\section{ACKNOWLEDGMENT}

We are grateful for the financial support from the University of Arizona for this work. We thanks Prof Tomat, Prof Huxter and Prof Njardarson for helpful discussion.

\section{REFERENCES}

(1) For selected reviews, see: (a) Prier, C. K.; Rankic, D. A.; MacMillan, D. W. C. Visible Light Photoredox Catalysis with Transition Metal Complexes: Applications in Organic Synthesis. Chem. Rev. 2013, 113, 5322-5363. (b) Schultz, D. M.; Yoon, T. P. Solar Synthesis: Prospects in Visible Light Photocatalysis. Science 2014, 343, 1239176. (c) Skubi, K. L.; Blum, T. R.; Yoon, T. P. Dual Catalysis Strategies in Photochemical Synthesis. Chem. Rev. 2016, 116, 10035-10074. (d) Hopkinson, M. N.; Tlahuext-Aca, A.; Glorius, F. Merging Visible Ligh Photoredox and Gold Catalysis. Acc. Chem. Res. 2016, 49, 2261-2272. (e) Lang, X.; Zhao, J.; Chen, X. Cooperative Photoredox Catalysis. Chem. Soc. Rev. 2016, 45, 3026-3038. (f) Twilton, J.; Le, C. C.; Zhang, P.; Shaw, M. H.; Evans, R. W.; MacMillan, D. W. C. The Merger of Transition Metal and Photocatalysis. Nat. Rev. Chem. 2017, 1, 0052. (g) Parasram, M.; Gevorgyan, V. Visible Light-Induced Transition MetalCatalyzed Transformations: Beyond Conventional Photosensitizers. Chem
Soc. Rev. 2017, 46, 6227-6240. (h) Marzo, L.; Pagire, S. K.; Reiser, O. König, B. Visible-Light Photocatalysis: Does It Make a Difference in Organic Synthesis? Angew. Chem., Int. Ed. 2018, 57, 10034-10072.

(2) Lowry, M. S.; Goldsmith, J. I.; Slinker, J. D.; Rohl, R.; Pascal, R. A.; Malliaras, G. G.; Bernhard, S. Single-Layer Electroluminescent Devices and Photoinduced Hydrogen Production from an Ionic Iridium(III) Complex. Chem. Mater. 2005, 17, 5712-5719.

(3) (a) Juris, A.; Balzani, V.; Belser, P.; von Zelewsky, A. Characterization of the Excited State Properties of Some New Photosensitizers of the Ruthenium (Polypyridine) Family. Helv. Chim. Acta 1981, 64, 2175-2182. (b) Kalyanasundaram, K. Photophysics, Photochemistry and Solar Energy Conversion with Tris(Bipyridyl)Ruthenium(II) and Its Analogues. Coord. Chem. Rev. 1982, 46, 159-244.

(4) (a) Romero, N. A.; Nicewicz, D. A. Organic Photoredox Catalysis. Chem. Rev. 2016, 116, 10075-10166. (b) Nicewicz, D. A.; Nguyen, T. M. Recent Applications of Organic Dyes as Photoredox Catalysts in Organic Synthesis. ACS Catal. 2014, 4, 355-360.

(5) Fukuzumi, S.; Kotani, H.; Ohkubo, K.; Ogo, S.; Tkachenko, N. V.; Lemmetyinen, H. Electron-Transfer State of 9-Mesityl-10Methylacridinium Ion with a Much Longer Lifetime and Higher Energy Than That of the Natural Photosynthetic Reaction Center. J. Am. Chem. Soc. 2004, 126, 1600-1601.

(6) For selected examples, see: (a) Hamilton, D. S.; Nicewicz, D. A Direct Catalytic Anti-Markovnikov Hydroetherification of Alkenols. $J$. Am. Chem. Soc. 2012, 134, 18577-18580. (b) Romero, N. A.; Margrey, K. A.; Tay, N. E.; Nicewicz, D. A. Site-Selective Arene C-H Amination via Photoredox Catalysis. Science 2015, 349, 1326-1330.

(7) (a) Neumann, M.; Füldner, S.; König, B.; Zeitler, K. Metal-Free, Cooperative Asymmetric Organophotoredox Catalysis with Visible Light. Angew. Chem., Int. Ed. 2011, 50, 951-954. (b) Hari, D. P.; König, B. Synthetic Applications of Eosin Y in Photoredox Catalysis. Chem. Commun. 2014, 50, 6688-6699. (c) Srivastava, V.; Singh, P. P. Eosin Y Catalysed Photoredox Synthesis: A Review. RSC Adv. 2017, 7, $31377-$ 31392 .

(8) (a) Ham, W. T.; Mueller, H. A.; Sliney, D. H. Retinal Sensitivity to Short Wavelength Light. Nature 1976, 260, 153-155. (b) Revell, V. L.; Skene, D. J. Light-Induced Melatonin Suppression in Humans with Polychromatic and Monochromatic Light. Chronobiol. Int. 2007, 24, 1125-1137. (c) Kuse, Y.; Ogawa, K.; Tsuruma, K.; Shimazawa, M.; Hara, H. Damage of Photoreceptor-Derived Cells in Culture Induced by Light Emitting Diode-Derived Blue Light. Sci. Rep. 2014, 4, 5223.

(9) Szaciłowski, K.; Macyk, W.; Drzewiecka-Matuszek, A.; Brindell, M.; Stochel, G. Bioinorganic Photochemistry: Frontiers and Mechanisms. Chem. Rev. 2005, 105, 2647-2694.

(10) For selected examples, see: (a) Fülöp, A.; Peng, X.; Greenberg, M M.; Mokhir, A. A Nucleic Acid-Directed, Red Light-Induced Chemical Reaction. Chem. Commun. 2010, 46, 5659-5661. (b) Zhang, H.; Trout, W S.; Liu, S.; Andrade, G. A.; Hudson, D. A.; Scinto, S. L.; Dicker, K. T.; Li, Y.; Lazouski, N.; Rosenthal, J.; Thorpe, C.; Jia, X.; Fox, J. M. Rapid Bioorthogonal Chemistry Turn-on through Enzymatic or Long Wavelength Photocatalytic Activation of Tetrazine Ligation. J. Am. Chem. Soc. 2016, 138, 5978-5983. (c) Carling, C. J.; Olejniczak, J.; FoucaultCollet, A.; Collet, G.; Viger, M. L.; Nguyen Huu, V. A.; Duggan, B. M.; Almutairi, A. Efficient Red Light Photo-Uncaging of Active Molecules in Water upon Assembly into Nanoparticles. Chem. Sci. 2016, 7, 2392-2398.

(11) (a) Cocquet, G.; Ferroud, C.; Guy, A. A Mild and Efficient Procedure for Ring-Opening Reactions of Piperidine and Pyrrolidine Derivatives by Single Electron Transfer Photooxidation. Tetrahedron 2000, 56, 2975-2984. (b) Cocquet, G.; Ferroud, C.; Simon, P.; Taberna, P. L. Single Electron Transfer Photoinduced Oxidation of Piperidine and Pyrrolidine Derivatives to the Corresponding Lactams. J. Chem. Soc. Perkin Trans. 2 2000, 1147-1153.

(12) For selected examples, see: (a) Pitre, S. P.; McTiernan, C. D. Ismaili, H.; Scaiano, J. C. Metal-Free Photocatalytic Radical Trifluoromethylation Utilizing Methylene Blue and Visible Light Irradiation. ACS Catal. 2014, 4, 2530-2535. (b) Kalaitzakis, D.; Kouridaki, A.; Noutsias, D.; Montagnon, T.; Vassilikogiannakis, G. Methylene Blue as a Photosensitizer and Redox Agent: Synthesis of 5Hydroxy-1H-Pyrrol-2(5H)-Ones from Furans. Angew. Chem., Int. Ed. 2015, 54, 6283-6287. (c) Jiang, H.; Mao, G.; Wu, H.; An, Q.; Zuo, M.; Guo, W.; Xu, C.; Sun, Z.; Chu, W. Synthesis of Dibenzocycloketones by 
Acyl Radical Cyclization from Aromatic Carboxylic Acids Using Methylene Blue as a Photocatalyst. Green Chem. 2019, 21, 5368-5373.

(13) (a) Lee, J.; Papatzimas, J. W.; Bromby, A. D.; Gorobets, E.; Derksen, D. J. Thiaporphyrin-Mediated Photocatalysis Using Red Light. RSC Adv. 2016, 6, 59269-59272. (b) Matsuzaki, K.; Hiromura, T.; Tokunaga, E.; Shibata, N. Trifluoroethoxy-Coated Subphthalocyanine Affects Trifluoromethylation of Alkenes and Alkynes Even under LowEnergy Red-Light Irradiation. ChemistryOpen 2017, 6, 226-230. (c) Yerien, D. E.; Cooke, M. V.; García Vior, M. C.; Barata-Vallejo, S.; Postigo, A. Radical Fluoroalkylation Reactions of (Hetero)Arenes and Sulfides under Red Light Photocatalysis. Org. Biomol. Chem. 2019, 17, 3741-3746.

(14) Ravetz, B. D.; Pun, A. B.; Churchill, E. M.; Congreve, D. N.; Rovis, T.; Campos, L. M. Photoredox Catalysis Using Infrared Light via Triplet Fusion Upconversion. Nature 2019, 565, 343-346.

(15) Ravetz, B. D.; Tay, N. E. S.; Joe, C. L.; Sezen-Edmonds, M.; Schmidt, M. A.; Tan, Y.; Janey, J. M.; Eastgate, M. D.; Rovis, T. SpinForbidden Excitation Enables Infrared Photoredox Catalysis. 2020, DOI: 10.26434/chemrxiv.12124215.v1.

(16) (a) Laursen, B. W.; Krebs, F. C. Synthesis of a Triazatriangulenium Salt. Angew. Chem., Int. Ed. 2000, 39, 3432-3434. (b) Delgado, I. H.; Pascal, S.; Wallabregue, A.; Duwald, R.; Besnard, C.; Guénée, L.; Nançoz, C.; Vauthey, E.; Tovar, R. C.; Lunkley, J. L.; Muller, G.; Lacour, J. Functionalized Cationic [4]Helicenes with Unique Tuning of Absorption, Fluorescence and Chiroptical Properties up to the Far-Red Range. Chem. Sci. 2016, 7, 4685-4693.

(17) Sørensen, T. J.; Nielsen, M. F.; Laursen, B. W. Synthesis and Stability of $N, N^{\prime}$-Dialkyl-1,13-Dimethoxyquinacridinium (DMQA+): A [4]Helicene with Multiple Redox States. ChemPlusChem 2014, 79, 10301035 .

(18) (a) Mei, L.; Veleta, J. M.; Bloch, J.; Goodman, H. J.; PierceNavarro, D.; Villalobos, A.; Gianetti, T. L. Tunable Carbocation-Based Redox Active Ambiphilic Ligands: Synthesis, Coordination and Characterization. Dalton Trans. 2020, DOI: 10.1039/d0dt00419g. (b) Duwald, R.; Pascal, S.; Bosson, J.; Grass, S.; Besnard, C.; Bürgi, T.; Lacour, J. Enantiospecific Elongation of Cationic Helicenes by Electrophilic Functionalization at Terminal Ends. Chem. - Eur. J. 2017, $23,13596-13601$

(19) (a) Bosson, J.; Gouin, J.; Lacour, J. Cationic Triangulenes and Helicenes: Synthesis, Chemical Stability, Optical Properties and Extended Applications of These Unusual Dyes. Chem. Soc. Rev. 2014, 43, 2824 2840. (b) Kel, O.; Fürstenberg, A.; Mehanna, N.; Nicolas, C.; Laleu, B.; Hammarson, M.; Albinsson, B.; Lacour, J.; Vauthey, E. Chiral Selectivity in the Binding of [4]Helicene Derivatives to Double-Stranded DNA. Chem. - Eur. J. 2013, 19, 7173-7180.

(20) Nicolas, C.; Herse, C.; Lacour, J. Catalytic Aerobic Photooxidation of Primary Benzylic Amines Using Hindered Acridinium Salts. Tetrahedron Lett. 2005, 46, 4605-4608.

(21) Kalyani, D.; McMurtrey, K. B.; Neufeldt, S. R.; Sanford, M. S. Room-Temperature C-H Arylation: Merger of Pd-Catalyzed C-H Functionalization and Visible-Light Photocatalysis. J. Am. Chem. Soc. 2011, 133, 18566-18569.

(22) (a) Allongue, P.; Delamar, M.; Desbat, B.; Fagebaume, O.; Hitmi, R.; Pinson, J.; Savéant, J. M. Covalent Modification of Carbon Surfaces by Aryl Radicals Generated from the Electrochemical Reduction of Diazonium Salts. J. Am. Chem. Soc. 1997, 119, 201-207. (b) Andrieux, C. P.; Pinson, J. The Standard Redox Potential of the Phenyl Radical/Anion Couple. J. Am. Chem. Soc. 2003, 125, 14801-14806.

(23) For selected examples, see: (a) Zou, Y. Q.; Chen, J. R.; Liu, X. P.; Lu, L. Q.; Davis, R. L.; Jørgensen, K. A.; Xiao, W. J. Highly Efficient Aerobic Oxidative Hydroxylation of Arylboronic Acids: Photoredox Catalysis Using Visible Light. Angew. Chem., Int. Ed. 2012, 51, 784-788. (b) Pitre, S. P.; McTiernan, C. D.; Ismaili, H.; Scaiano, J. C. Mechanistic Insights and Kinetic Analysis for the Oxidative Hydroxylation of Arylboronic Acids by Visible Light Photoredox Catalysis: A Metal-Free Alternative. J. Am. Chem. Soc. 2013, 135, 13286-13289. (c) Yu, X.; Cohen, S. M. Photocatalytic Metal-Organic Frameworks for the Aerobic Oxidation of Arylboronic Acids. Chem. Commun. 2015, 51, 9880-9883. (d) Xie, H. Y.; Han, L. S.; Huang, S.; Lei, X.; Cheng, Y.; Zhao, W.; Sun, H.; Wen, X.; Xu, Q. L. N-Substituted 3(10H)-Acridones as Visible-Light, Water-Soluble Photocatalysts: Aerobic Oxidative Hydroxylation of Arylboronic Acids. J. Org. Chem. 2017, 82, 5236-5241.
(24) (a) Pavlishchuk, V. V; Addison, A. W. Conversion Constants for Redox Potentials Measured versus Different Reference Electrodes in Acetonitrile Solutions at $25{ }^{\circ} \mathrm{C}$. Inorg. Chim. Acta 2000, 298, 97-102. (b) Barbante, G. J.; Kebede, N.; Hindson, C. M.; Doeven, E. H.; Zammit, E. M.; Hanson, G. R.; Hogan, C. F.; Francis, P. S. Control of Excitation and Quenching in Multi-Colour Electrogenerated Chemiluminescence Systems through Choice of Co-Reactant. Chem. - Eur. J. 2014, 20, 14026-14031. (c) Schwarz, J.; König, B. Metal-Free, Visible-Light-Mediated, Decarboxylative Alkylation of Biomass-Derived Compounds. Green Chem. 2016, 18, 4743-4749.

(25) Wood, P. M. The Redox Potential of the System OxygenSuperoxide. FEBS Lett. 1974, 44, 22-24.

(26) For selected examples, see: (a) Finney, L. C.; Mitchell, L. J.; Moody, C. J. Visible Light Mediated Oxidation of Benzylic spз C-H Bonds Using Catalytic 1,4-Hydroquinone, or Its Biorenewable Glucoside, Arbutin, as a Pre-Oxidant. Green Chem. 2018, 20, 2242-2249. (b) Zhang, Y.; Riemer, D.; Schilling, W.; Kollmann, J.; Das, S. Visible-LightMediated Efficient Metal-Free Catalyst for $\alpha$-Oxygenation of Tertiary Amines to Amides. ACS Catal. 2018, 8, 6659-6664. (c) Ren, L.; Yang, M. M.; Tung, C. H.; Wu, L. Z.; Cong, H. Visible-Light Photocatalysis Employing Dye-Sensitized Semiconductor: Selective Aerobic Oxidation of Benzyl Ethers. ACS Catal. 2017, 7, 8134-8138.

(27) For selected examples, see: (a) Nguyen, J. D.; Tucker, J. W.; Konieczynska, M. D.; Stephenson, C. R. J. Intermolecular Atom Transfer Radical Addition to Olefins Mediated by Oxidative Quenching of Photoredox Catalysts. J. Am. Chem. Soc. 2011, 133, 4160-4163. (b) Wallentin, C. J.; Nguyen, J. D.; Finkbeiner, P.; Stephenson, C. R. J. Visible Light-Mediated Atom Transfer Radical Addition via Oxidative and Reductive Quenching of Photocatalysts. J. Am. Chem. Soc. 2012, 134, 8875-8884. (c) Pirtsch, M.; Paria, S.; Matsuno, T.; Isobe, H.; Reiser, O. $\left[\mathrm{Cu}(\mathrm{Dap})_{2} \mathrm{Cl}\right]$ as an Efficient Visible-Light-Driven Photoredox Catalyst in Carbon-Carbon Bond-Forming Reactions. Chem. - Eur. J. 2012, 18, 73367340. (d) Paria, S.; Pirtsch, M.; Kais, V.; Reiser, O. Visible-Light-Induced Intermolecular Atom-Transfer Radical Addition of Benzyl Halides to Olefins: Facile Synthesis of Tetrahydroquinolines. Synthesis 2013, 45, 2689-2698. (e) Rawner, T.; Lutsker, E.; Kaiser, C. A.; Reiser, O. The Different Faces of Photoredox Catalysts: Visible-Light-Mediated Atom Transfer Radical Addition (ATRA) Reactions of Perfluoroalkyl Iodides with Styrenes and Phenylacetylenes. ACS Catal. 2018, 8, 3950-3956.

(28) Tlahuext-Aca, A.; Hopkinson, M. N.; Sahoo, B.; Glorius, F. Dual Gold/Photoredox-Catalyzed C(sp)-H Arylation of Terminal Alkynes with Diazonium Salts. Chem. Sci. 2016, 7, 89-93. 
Table of Content
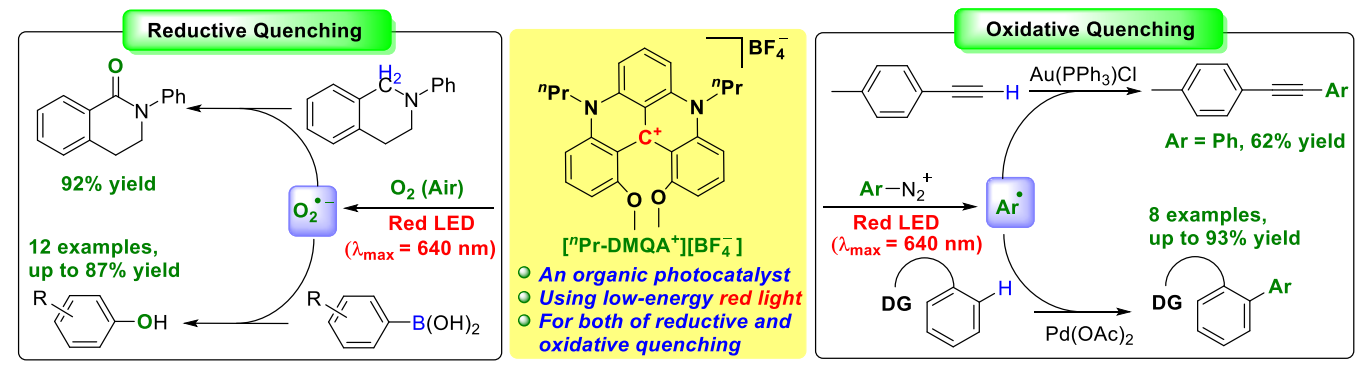


\title{
Supporting Information
}

\section{Helical Carbenium Ion: A Versatile Organic Photoredox Catalyst in Red-Light- Mediated Reactions}

\author{
Liangyong Mei, José M. Veleta, and Thomas L. Gianetti*
}

\begin{abstract}
Department of Chemistry and Biochemistry, University of Arizona, Tucson, Arizona 85721, United States

tgianetti@arizona.edu.
\end{abstract}

\section{CONTENTS}

1. General remarks S2

2. Synthesis and characterization of dimethoxyquinacridinium (DMQA+) tetrafluoroborate 3..S3

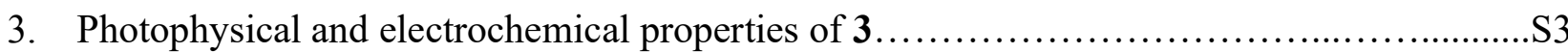

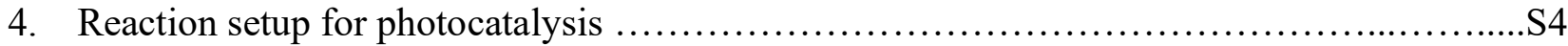

5. Typical procedure for red-light-mediated dual $\mathrm{Pd} / n \mathrm{Pr}-\mathrm{DMQA}+-$ catalyzed $\mathrm{C}-\mathrm{H}$ arylation......S5

6. Optimization of red-light-mediated dual $\mathrm{Pd} / n \mathrm{Pr}-\mathrm{DMQA}+-$ catalyzed $\mathrm{C}-\mathrm{H}$ arylation..............S8

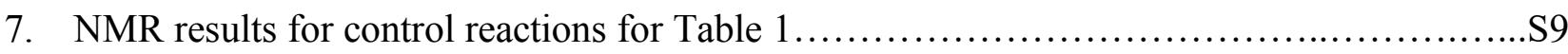

8. Typical procedure for ${ }_{n} \operatorname{Pr}-\mathrm{DMQA}+-$ catalyzed aerobic oxidative hydroxylation of arylboronic

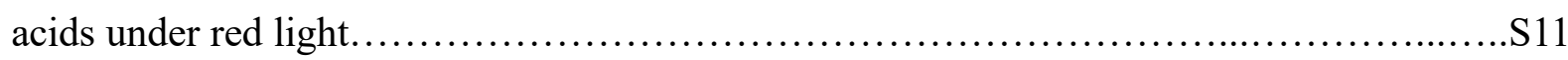

9. Optimization of ${ }_{n} \mathrm{Pr}-\mathrm{DMQA}+-$ catalyzed aerobic oxidative hydroxylation.....................S14

10. Experimental procedure for red-light-induced ${ }_{n} \mathrm{Pr}-\mathrm{DMQA}+-$ catalyzed oxygenation..............S14

11. Experimental procedure for red-light-induced ${ }_{n}$ Pr-DMQA+-catalyzed ATRA.......................S15

12. Optimization of red-light-induced ${ }_{n}$ Pr-DMQA+-catalyzed intermolecular ATRA..............S16

13. Proposed mechanism for red-light-induced ${ }_{n}$ Pr-DMQA+-catalyzed intermolecular ATRA...S18

14. Experimental procedure for red-light-mediated dual $\mathrm{Au} / n \operatorname{Pr}-\mathrm{DMQA}+-$ catalyzed $\mathrm{C}(\mathrm{sp})-\mathrm{H}$

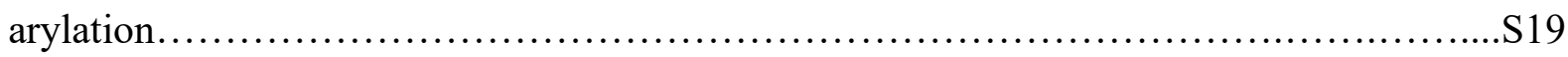

15. Optimization of red-light-mediated dual $\mathrm{Au} / n \mathrm{Pr}$-DMQA+-catalyzed $\mathrm{C}(\mathrm{sp})-\mathrm{H}$ arylation.......S20

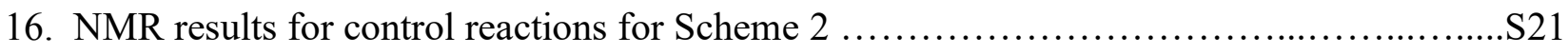

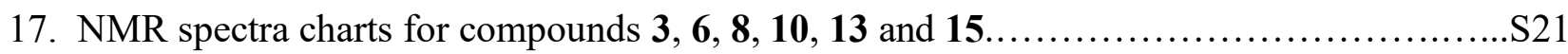

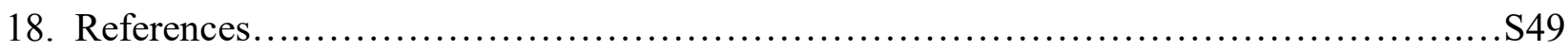




\section{General remarks}

Unless otherwise specified, all reactions were carried out in oven-dried (overnight) vials or Schlenk tubes with magnetic stirring in a glove box. ${ }_{1} \mathrm{H},{ }_{13} \mathrm{C}$ and ${ }_{19} \mathrm{~F}$ NMR spectra were recorded on Bruker Avance III-400 MHz or DRX-500 MHz spectrometers in appropriate solvents using TMS as internal standard or the solvent signals as secondary standards. The chemical shifts are shown in $\delta$ scales. Multiplicities of $1 \mathrm{H}$ NMR signals are designated as s (singlet), d (doublet), dd (doublet of doublet), dt (doublet of triplet), $\mathrm{t}$ (triplet), quin (quintet), m (multiplet), etc.. Compounds were named using ChemDraw and assignments of NMR spectra were done using MestReNova. All chemicals and solvents were purchased from Sigma Aldrich, Fisher Scientific, or VWR. Organic solvents used were dried by a standard solvent purification system (J. C. Meyer or Vigor Solvent Systems). Commercially obtained reagents were used without further purification. All reactions were monitored by TLC silica gel 60 F254 (EMD Millipore). Flash column chromatography was carried out using SiliaFlash silica gels F60, 40-63 $\mu \mathrm{m}, 60 \AA$ (SiliCycle) at increased pressure. All reactions were performed under $\mathrm{N}_{2}$ using standard Schlenk techniques or in a glove box (Mbraun glove box).

Substrates $\mathbf{4 b}, 1$ 4c, 2 and $\mathbf{4 d} 3$ were prepared according to the previously reported procedures. Aryl diazonium tetrafluoroborates $\mathbf{5 a - 5 c}$ were prepared according to the previously reported procedures. 4 2-Phenyl-3,4-dihydroisoquinolin-1(2H)-one 9 was prepared according to the previously reported procedure.5 $\mathrm{Au}\left(\mathrm{PPh}_{3}\right) \mathrm{Cl}$ was prepared from $\mathrm{PPh}_{3}$ and $\mathrm{AuCl}\left(\mathrm{SMe}_{2}\right)$ according to the previously reported procedure. 6 


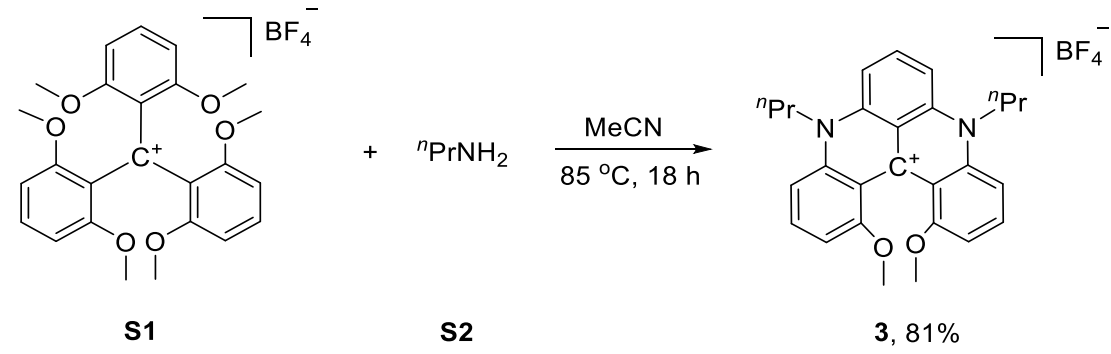

3 was synthesized according to a modified procedure from previous paper.7

A solution of $\mathbf{S 1}$ (1.02 g, 2 mmol, 1.0 equiv.) and $\mathbf{S 2}$ (2.95 g, $50 \mathrm{mmol}, 25.0$ equiv.) in MeCN (20 $\mathrm{mL}$ ) was stirred at $85{ }^{\circ} \mathrm{C}$ for 18 hours. A dark green solution was formed. The mixture was cooled to room temperature, transferred to a $250 \mathrm{~mL}$ round bottom flask and a large excess of $\mathrm{Et} 2 \mathrm{O}(200$ $\mathrm{mL}$ ) was added to crash out the crude product 3 as a dark green solid (924 mg). After layering in $\mathrm{DCM} / \mathrm{MeCN} / \mathrm{MeOH} / \mathrm{Et}_{2} \mathrm{O}$ for 2 days, $811 \mathrm{mg}$ of pure product 3 was obtained as a dark green solid after filtration.

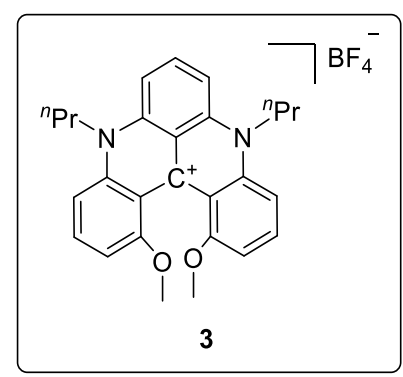

3: Yield: (811 mg, 81\%). A dark green solid.1H NMR (400 MHz, DMSO- $d 6) \delta 8.24$ (dd, $J=8.4$, $8.4 \mathrm{~Hz}, 1 \mathrm{H}, \mathrm{ArH}), 7.95(\mathrm{dd}, J=8.4,8.4 \mathrm{~Hz}, 2 \mathrm{H}, \mathrm{ArH}), 7.68(\mathrm{~d}, J=8.4 \mathrm{~Hz}, 2 \mathrm{H}, \mathrm{ArH}), 7.60(\mathrm{~d}, J=$ $8.4 \mathrm{~Hz}, 2 \mathrm{H}, \mathrm{ArH}), 7.01$ (d, J=8.4 Hz, 2H, ArH), 4.77-4.68 (m, 2H, CH2), 4.51-4.42 (m, 2H, $\left.\mathrm{CH}_{2}\right)$, $3.71(\mathrm{~s}, 6 \mathrm{H}, \mathrm{OMe}), 2.04-1.92\left(\mathrm{~m}, 4 \mathrm{H}, \mathrm{CH}_{2}\right), 1.15\left(\mathrm{t}, J=7.2 \mathrm{~Hz}, 6 \mathrm{H}, \mathrm{CH}_{3}\right) .{ }_{13} \mathrm{C} \mathrm{NMR}(101 \mathrm{MHz}$, DMSO-d6) $\delta 159.12,141.78,141.59,138.22,137.16,136.71,118.58,112.27,107.69,105.09$, 103.01, 55.67, 50.56, 19.38, 10.82. 19F NMR (376 MHz, DMSO-d6) $\delta-148.25,-148.30$.

\section{Photophysical and electrochemical properties of 3}

The photophysical properties of $\mathbf{3}$ have been well-documented in the previous literature, 7,8 which is featured with peak absorption at $616 \mathrm{~nm}$ and peak emission at $667 \mathrm{~nm}$. The electrochemical behavior of $\mathbf{3}$ has been well-studied by cyclic voltammetry (CV) in previously reports, 8,9 which was featured 
with a quasi-reversible oxidation of $\mathrm{C}+$ to $\mathrm{C} \cdot++\left(\mathrm{E}_{1 / 2}(\mathrm{C} \cdot++/ \mathrm{C}+)\right)$ at $1.32 \mathrm{~V}$ versus the saturated calomel electrode $(\mathrm{SCE})$ and a fully reversible reduction of $\mathrm{C}+$ to $\mathrm{C} \cdot\left(\mathrm{E}_{1 / 2}(\mathrm{C}+/ \mathrm{C} \cdot)\right)$ at $-0.78 \mathrm{~V}$ versus $\mathrm{SCE}$ in acetonitrile.

Based on the reported literature, 10 excitation energy $\left(\mathrm{E}_{0,0}\right)$ is estimated by calculating the energy of the wavelength at which the compound's UV-Vis absorption and emission spectra overlap or of the wavelength at the midpoint between absorption and emission maxima. Then, excited state reduction potential $\mathrm{E}_{1 / 2}(\mathrm{C}+* / \mathrm{C} \cdot)$ is calculated by $\mathrm{E}_{1 / 2}(\mathrm{C}+* / \mathrm{C} \cdot)=\mathrm{E}_{1 / 2}(\mathrm{C}+/ \mathrm{C} \cdot)+\mathrm{E}_{0,0}$; while excited state oxidation potential $\mathrm{E}_{1 / 2}\left(\mathrm{C} \cdot++/ \mathrm{C}+*^{*}\right)$ is calculated by $\mathrm{E}_{1 / 2}(\mathrm{C} \cdot++/ \mathrm{C}+*)=\mathrm{E}_{1 / 2}(\mathrm{C} \cdot++/ \mathrm{C}+)-\mathrm{E}_{0,0}$. Thus, we can obtain the corresponding photophysical and electrochemical properties of $\mathbf{3}$ (Table S1).

Absorption $\lambda_{\max }=616 \mathrm{~nm}$

Emission $\lambda_{\max }=667 \mathrm{~nm}$

Midpoint of absorption and emission maxima $\lambda=642 \mathrm{~nm}$

$\mathrm{E}_{0,0}=1240 / 642 \mathrm{eV}=1.93 \mathrm{eV}$

$\mathrm{E}_{1 / 2}(\mathrm{C}+* / \mathrm{C} \cdot)=-0.78+1.93 \mathrm{~V}=1.15 \mathrm{~V}$

$\mathrm{E}_{1 / 2}(\mathrm{C} \cdot++/ \mathrm{C}+*)=1.32-1.93 \mathrm{~V}=-0.61 \mathrm{~V}$

\begin{tabular}{|c|c|c|c|c|c|c|c|c|}
\hline PC & $\begin{array}{l}E_{0,0} \\
(e V)^{a}\end{array}$ & $\begin{array}{c}E_{1 / 2}\left(C^{+++} / C^{+}\right) \\
(V)^{b}\end{array}$ & $\begin{array}{c}E_{1 / 2}\left(C^{+} / C\right) \\
(V)^{b}\end{array}$ & $\begin{array}{c}E_{1 / 2}\left(C^{++} / C^{+*}\right) \\
(V)^{c}\end{array}$ & $\begin{array}{c}E_{1 / 2}\left(C^{+*} / C \cdot\right) \\
(V)^{C}\end{array}$ & $\begin{array}{c}\text { excited state lifetime } \\
\tau(\mathrm{ns})^{\mathrm{d}}\end{array}$ & $\begin{array}{c}\text { absorption } \\
\lambda_{\max }(\mathrm{nm})\end{array}$ & $\begin{array}{l}\text { emission } \\
\lambda_{\max }(\mathrm{nm})\end{array}$ \\
\hline 3 & 1.93 & +1.32 & -0.78 & -0.61 & +1.15 & 5.5 & 616 & 667 \\
\hline
\end{tabular}

Table S1. Photophysical and electrochemical properties of $\mathbf{3}$

\section{Reaction setup for photocatalysis}

The reaction was set up by referring to MacMillan group.11

Two $25 \mathrm{~mL}$ Schlenk tubes are placed in a water bath (Pyrex crystallizing dish, $125 \mathrm{x}$ 65mm, No. 3140) at the center of a stir plate. Two parallel Red LED lamps (KSPR160L-640-C Red LED 640nm Photoredox Light customized wavelength, Kessil LED Lights) are placed perpendicular to the sidewall of Schlenk tubes, so that the two tubes can be equally exposed to the LEDs. The stir plate/water bath/LEDs are surrounded by an open-top cardboard box covered with aluminum foil to increase the light reflections. A fan (Honeywell, TurboForce Power HT900) over the water bath is always turned on when the reaction is running. 
Note: The combination of an overhead fan and a water bath is to offset the heat generated from the LED lights and stabilize reaction temperature for reproducible results. The water bath needs to be refilled with room temperature deionized water every 12-18 hours. With the above setup, the reaction temperature can be maintained at $21-23{ }^{\circ} \mathrm{C}$ during the reaction for us.

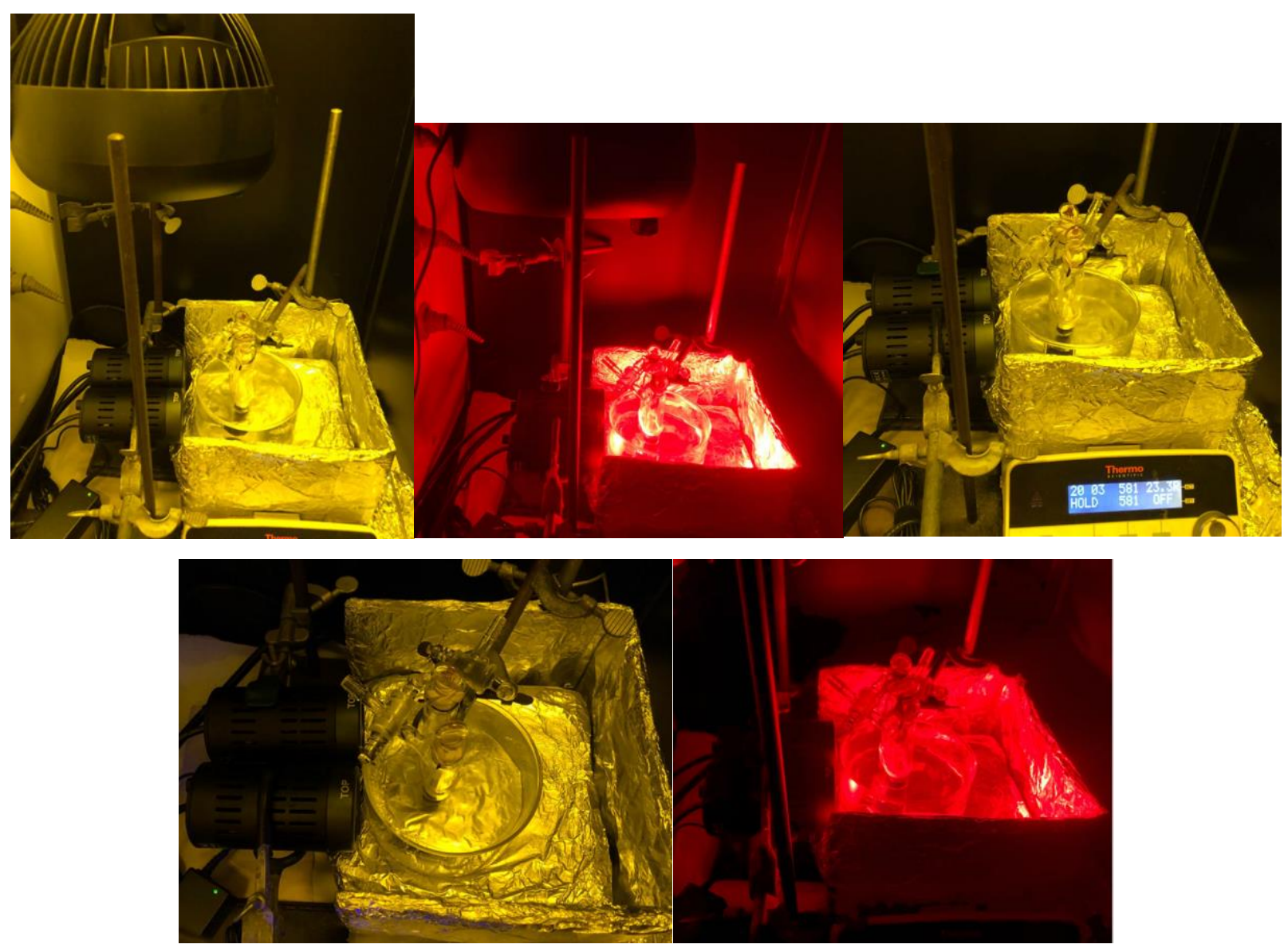

Figure S1. Reaction setup for photocatalysis.

\section{Typical procedure for red-light-mediated dual Pd/nPr-DMQA+-catalyzed $\mathrm{C}-\mathrm{H}$ arylation}

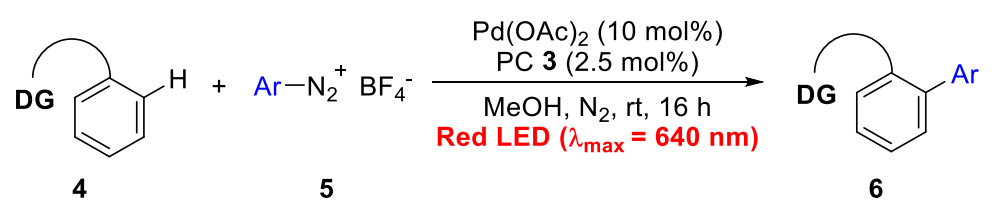

In a $\mathrm{N}_{2}$ glove box, $\mathrm{Pd}(\mathrm{OAc}) 2$ (4.5 mg, $\left.0.02 \mathrm{mmol}, 10 \mathrm{~mol} \%\right), 3$ (2.5 mg, $\left.0.005 \mathrm{mmol}, 2.5 \mathrm{~mol} \%\right)$, the substrate 4 ( $0.2 \mathrm{mmol}, 1.0$ equiv. $)$ and the aryldiazonium salt 5 ( $0.8 \mathrm{mmol}, 4.0$ equiv. $)$ were added to an oven-dried (overnight) Schlenk tube containing a stirring bar, followed by adding degassed anhydrous $\mathrm{MeOH}(2.0 \mathrm{~mL}, 0.1 \mathrm{M})$. The Schlenk tube was then sealed, removed from the glove box 
and stirred at room temperature under red LED $\left(\lambda_{\max }=640 \mathrm{~nm}\right)$ irradiation. After 16 hours, the mixture was quenched with a saturated solution of $\mathrm{NaHCO}_{3}(2 \mathrm{~mL})$, followed by adding deionized water $(2 \mathrm{~mL})$. The crude reaction mixture was then extracted with ethyl acetate $(3 \times 10 \mathrm{~mL})$, and the combined organic layers were washed with brine $(20 \mathrm{~mL})$ and dried over anhydrous $\mathrm{Na}_{2} \mathrm{SO}_{4}$. After filtration, the solvent was removed under reduced pressure on RotaVap. The crude product was purified by flash chromatography (FC) on silica gel (eluent: Hexanes/EtOAc $=20 / 1 \sim 1 / 1$ ) to yield the desired product $\mathbf{6}$.

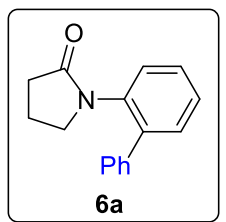

1-([1,1'-biphenyl]-2-yl)pyrrolidin-2-one (6a): Yield (44 mg, 93\%). A clear viscous oil. $\mathrm{Rf}_{\mathrm{f}}=0.2$ $($ Hexanes/EtOAc $=1 / 2) . \mathrm{FC}($ Hexanes/EtOAc $=1 / 1) .{ }_{1} \mathrm{H}$ NMR $\left(400 \mathrm{MHz}, \mathrm{C}_{6} \mathrm{D} 6\right) \delta$ 7.39-7.35 (m, 2H, ArH), 7.33 (dd, $J=8.0,1.6 \mathrm{~Hz}, 1 \mathrm{H}, \mathrm{ArH}), 7.21$ (dd, $J=7.6,1.6 \mathrm{~Hz}, 1 \mathrm{H}, \mathrm{ArH}), 7.17-7.04$ (m, $5 \mathrm{H}, \mathrm{ArH}), 2.78\left(\mathrm{t}, J=6.8 \mathrm{~Hz}, 2 \mathrm{H}, \mathrm{CH}_{2}\right), 2.04\left(\mathrm{t}, J=8.0 \mathrm{~Hz}, 2 \mathrm{H}, \mathrm{CH}_{2}\right), 1.19(\mathrm{tt}, J=6.8,8.0 \mathrm{~Hz}, 2 \mathrm{H}$, $\mathrm{CH}_{2}$ ). ${ }_{13} \mathrm{C}$ NMR (101 MHz, C6 6 6) $\delta 174.18,140.13,140.08,137.67,130.98,129.18,128.82,128.63$, 128.48, 127.74, 127.64, 49.70, 31.11, 19.06. $1 \mathrm{H} \mathrm{NMR}\left(400 \mathrm{MHz}, \mathrm{CDCl}_{3}\right) \delta$ 7.43-7.31 (m, 9H, $\left.\mathrm{ArH}\right)$, $3.21\left(\mathrm{t}, J=6.8 \mathrm{~Hz}, 2 \mathrm{H}, \mathrm{CH}_{2}\right), 2.42\left(\mathrm{t}, J=8.0 \mathrm{~Hz}, 2 \mathrm{H}, \mathrm{CH}_{2}\right), 1.87\left(\mathrm{tt}, J=6.8,8.0 \mathrm{~Hz}, 2 \mathrm{H}, \mathrm{CH}_{2}\right) .{ }_{13} \mathrm{C}$ NMR $\left(101 \mathrm{MHz}, \mathrm{CDCl}_{3}\right) \delta 175.70,139.73,139.22,136.43,130.92,128.65,128.51,128.49,128.45$, $128.12,127.67,50.26,31.30,19.08$.

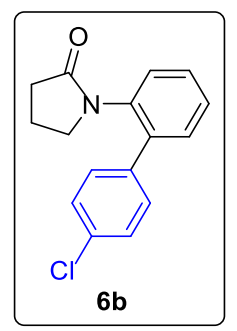

1-(4'-chloro-[1,1'-biphenyl]-2-yl)pyrrolidin-2-one (6b): Yield (50 mg, 92\%). A light yellow solid. $\mathrm{R}_{\mathrm{f}}=0.2($ Hexanes/EtOAc $=1 / 2) . \mathrm{FC}($ Hexanes/EtOAc $=1 / 1) .{ }_{1} \mathrm{H}$ NMR $\left(400 \mathrm{MHz}, \mathrm{C}_{6} \mathrm{D} 6\right) \delta 7.24$ $(\mathrm{dd}, J=8.0,1.2 \mathrm{~Hz}, 1 \mathrm{H}, \mathrm{ArH}), 7.15-7.04(\mathrm{~m}, 7 \mathrm{H}, \mathrm{ArH}), 2.73(\mathrm{t}, J=6.8 \mathrm{~Hz}, 2 \mathrm{H}, \mathrm{CH} 2), 2.00$ (t, $J=$ $\left.8.0 \mathrm{~Hz}, 2 \mathrm{H}, \mathrm{CH}_{2}\right), 1.18\left(\mathrm{tt}, J=6.8,8.0 \mathrm{~Hz}, 2 \mathrm{H}, \mathrm{CH}_{2}\right) .{ }_{13} \mathrm{C}$ NMR $\left(101 \mathrm{MHz}, \mathrm{C}_{6} \mathrm{D}_{6}\right) \delta 174.18,138.86$, $138.46,137.53,133.78,130.79,130.21,129.01,128.82,128.81,49.77,31.03,19.01$. 1 H NMR (400 $\mathrm{MHz}_{\mathrm{CDCl}}$ ) $\delta$ 7.44-7.30 (m, 8H, ArH), 3.26 (t, $\left.J=6.8 \mathrm{~Hz}, 2 \mathrm{H}, \mathrm{CH}_{2}\right), 2.43\left(\mathrm{t}, J=8.0 \mathrm{~Hz}, 2 \mathrm{H}, \mathrm{CH}_{2}\right)$, $1.92\left(\mathrm{tt}, J=6.8,8.0 \mathrm{~Hz}, 2 \mathrm{H}, \mathrm{CH}_{2}\right) .{ }_{13} \mathrm{C} \mathrm{NMR}\left(126 \mathrm{MHz}, \mathrm{CDCl}_{3}\right) \delta 175.83,138.71,137.78,136.49$, $133.87,130.85,129.88,129.10,128.79,128.58,128.34,128.33,50.33,31.13,18.95$. 


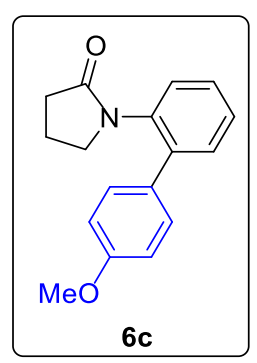

1-(4'-methoxy-[1,1'-biphenyl]-2-yl)pyrrolidin-2-one (6c): Yield (46 mg, 86\%). An orange viscous oil. $\mathrm{Rf}_{\mathrm{f}}=0.2($ Hexanes/EtOAc $=1 / 2) . \mathrm{FC}($ Hexanes/EtOAc $=1 / 1) .1 \mathrm{H} \mathrm{NMR}\left(400 \mathrm{MHz}, \mathrm{CDCl}_{3}\right) \delta$ 7.38-7.34 (m, 3H, ArH), 7.32-7.28 (m, 3H, ArH), 6.94-6.92 (m, 2H, ArH), $3.84(\mathrm{~s}, 3 \mathrm{H}), 3.22(\mathrm{t}, J=$ $\left.6.8 \mathrm{~Hz}, 2 \mathrm{H}, \mathrm{CH}_{2}\right), 2.44$ (t, $\left.J=8.0 \mathrm{~Hz}, 2 \mathrm{H}, \mathrm{CH}_{2}\right), 1.89$ (tt, $\left.J=6.8,8.0 \mathrm{~Hz}, 2 \mathrm{H}, \mathrm{CH}_{2}\right) .13 \mathrm{C}$ NMR (101 $\left.\mathrm{MHz}_{2} \mathrm{CDCl}_{3}\right) \delta 175.76,159.25,139.37,136.42,131.58,130.94,129.59,128.49,128.30,128.12$, $113.96,55.37,50.18,31.35,19.11$.

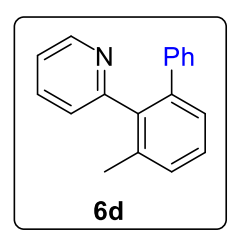

2-(3-methyl-[1,1'-biphenyl]-2-yl)pyridine (6d): Yield (36 mg, 73\%). A clear viscous oil. $\mathrm{R}_{\mathrm{f}}=0.4$ $($ Hexanes $/$ EtOAc $=5 / 1) . \mathrm{FC}($ Hexanes/EtOAc $=20 / 1) .1 \mathrm{H} \mathrm{NMR}\left(500 \mathrm{MHz}, \mathrm{CDCl}_{3}\right) \delta 8.63(\mathrm{ddd}, J$ $=5.0,2.0,1.0 \mathrm{~Hz}, 1 \mathrm{H}, \mathrm{ArH}), 7.44(\mathrm{ddd}, J=7.5,7.5,1.5 \mathrm{~Hz}, 1 \mathrm{H}, \mathrm{ArH}), 7.36(\mathrm{dd}, J=7.5,7.5 \mathrm{~Hz}$, 1H, ArH), 7.31-7.26 (m, 2H, ArH), 7.16-7.11 (m, 3H, ArH), 7.10-7.06 (m, 3H, ArH), 6.88 (ddd, $J$ $=7.5,7.5,1.5 \mathrm{~Hz}, 1 \mathrm{H}, \mathrm{ArH}), 2.19(\mathrm{~s}, 3 \mathrm{H}, \mathrm{Me}) .13 \mathrm{C} \mathrm{NMR}\left(101 \mathrm{MHz}, \mathrm{CDCl}_{3}\right) \delta 159.69,148.92$, $141.77,141.36,139.41,136.80,135.81,129.75,129.71,129.52,128.15,127.70,127.69,126.32$, $125.74,121.40,20.59$.

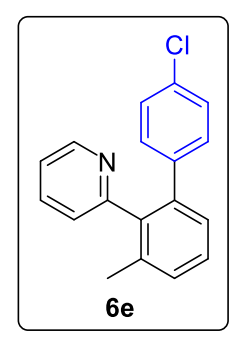

2-(4'-chloro-3-methyl-[1,1'-biphenyl]-2-yl)pyridine (6e): Yield (38 mg, 68\%). A pale yellow viscous oil. $\mathrm{Rf}_{\mathrm{f}}=0.4($ Hexanes/EtOAc $=5 / 1) . \mathrm{FC}($ Hexanes/EtOAc $=20 / 1) .{ }_{1} \mathrm{H} \mathrm{NMR}(400 \mathrm{MHz}$, $\left.\mathrm{CDCl}_{3}\right) \delta 8.62(\mathrm{ddd}, J=4.8,4.8,1.2 \mathrm{~Hz}, 1 \mathrm{H}, \mathrm{ArH}), 7.48(\mathrm{ddd}, J=7.6,7.6,2.0 \mathrm{~Hz}, 1 \mathrm{H}, \mathrm{ArH}), 7.38$ 7.28 (m, 2H, ArH), 7.23 (dd, J= 7.6, 1.2 Hz, 1H, ArH), 7.13-7.09 (m, 3H, ArH), 7.03-6.99 (m, 2H, ArH), 6.89 (ddd, $J=7.6,7.6,1.2 \mathrm{~Hz}, 1 \mathrm{H}, \mathrm{ArH}), 2.18$ (s, 3H, Me). ${ }_{13} \mathrm{C} \mathrm{NMR}\left(101 \mathrm{MHz}, \mathrm{CDCl}_{3}\right) \delta$ $159.40,149.11,140.26,140.07,139.42$, 136.97, 136.00, 132.46, 130.99, 129.83, 128.24, 127.91, $127.53,125.64,121.58,20.55$. 


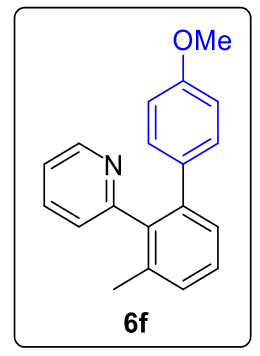

2-(4'-methoxy-3-methyl-[1,1'-biphenyl]-2-yl)pyridine (6f): Yield (33 mg, 60\%). A clear viscous oil. $\mathrm{Rf}_{\mathrm{f}}=0.4($ Hexanes/EtOAc $=5 / 1) . \mathrm{FC}($ Hexanes/EtOAc $=20 / 1) .{ }_{1} \mathrm{H} \mathrm{NMR}\left(400 \mathrm{MHz}, \mathrm{CDCl}_{3}\right) \delta 8.63$ (ddd, J = 4.8, 4.8, 1.2 Hz, 1H, ArH), 7.46 (ddd, J = 7.6, 7.6, $2.0 \mathrm{~Hz}, 1 \mathrm{H}, \mathrm{ArH}), 7.34$ (dd, J= 7.6, 7.6 $\mathrm{Hz}, 1 \mathrm{H}, \mathrm{ArH}), 7.28-7.23$ (m, 2H, ArH), 7.09 (ddd, J=7.6, 4.8, 1.2 Hz, 1H, ArH), 7.02-6.97 (m, 2H, ArH), 6.88 (d, J=8.0 Hz, 1H, ArH), 6.70-6.66 (m, 2H, ArH), 3.73 (s, 3H, OMe), 2.18 (s, 3H, Me). ${ }_{13} \mathrm{C}$ NMR $\left(101 \mathrm{MHz}, \mathrm{CDCl}_{3}\right) \delta 159.93,158.17,148.98,140.90,139.45,136.78,135.86,134.22$, $130.78,129.18,128.11,127.69,125.71,121.33,113.18,55.20,20.60$.

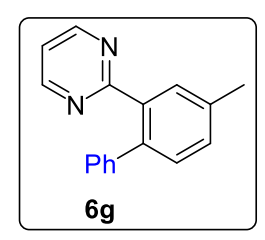

2-(4-methyl-[1,1'-biphenyl]-2-yl)pyrimidine (6g): Yield (28 mg, 57\%). A clear viscous oil. $\mathrm{R}_{\mathrm{f}}=0.3$ $($ Hexanes/EtOAc $=3 / 1) . \mathrm{FC}(\mathrm{Hexanes} / \mathrm{EtOAc}=15 / 1) .{ }_{1} \mathrm{H}$ NMR $\left(400 \mathrm{MHz}, \mathrm{CDCl}_{3}\right) \delta 8.63(\mathrm{~d}, J=$ $4.8 \mathrm{~Hz}, 2 \mathrm{H}, \mathrm{ArH}), 7.61(\mathrm{~d}, J=1.6 \mathrm{~Hz}, 1 \mathrm{H}, \mathrm{ArH}), 7.37$ (d, $J=7.6 \mathrm{~Hz}, 1 \mathrm{H}, \mathrm{ArH}), 7.32$ (dd, $J=7.6$, $1.6 \mathrm{~Hz}, 1 \mathrm{H}, \mathrm{ArH}), 7.24-7.18$ (m, 3H, ArH), 7.14-7.10 (m, 2H, ArH), 7.08 (dd, J=4.8, $4.8 \mathrm{~Hz}, 1 \mathrm{H}$, $\mathrm{ArH}), 2.46$ (s, 3H, Me). ${ }_{13} \mathrm{C} \mathrm{NMR}\left(101 \mathrm{MHz}, \mathrm{CDCl}_{3}\right) \delta 168.37,156.83,141.67,138.76,138.12$, $137.28,131.19,130.79,130.29,129.28,128.05,126.39,118.50,21.18$.

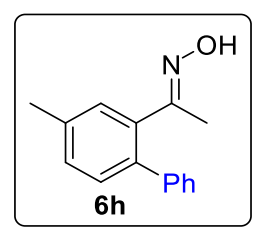

1-(4-methyl-[1,1'-biphenyl]-2-yl)ethan-1-one oxime (6h): Yield (29 mg, 64\%). A white solid. $\mathrm{R}_{\mathrm{f}}=$ $0.4($ Hexanes/EtOAc $=5 / 1)$. FC $\left(\right.$ Hexanes/EtOAc = 20/1). ${ }_{1} \mathrm{H} \mathrm{NMR}\left(400 \mathrm{MHz}, \mathrm{C}_{6} \mathrm{D}_{6}\right) \delta 8.69(\mathrm{~s}, 1 \mathrm{H}$, $\mathrm{OH}), 7.40-7.37$ (m, 2H, ArH), 7.32 (d, $J=2.0 \mathrm{~Hz}, 1 \mathrm{H}, \mathrm{ArH}), 7.13-7.03$ (m, 3H, ArH), 6.96 (dd, $J=$ 7.6, $2.0 \mathrm{~Hz}, 1 \mathrm{H}, \mathrm{ArH}), 2.09$ (s, 3H, Me), 1.78 (s, 3H, Me). 13C NMR (101 MHz, C6 6 6) $\delta 159.07$, 141.64, 138.32, 137.39, 137.27, 130.55, 130.44, 129.86, 129.37, 128.69, 127.31, 20.85, 16.30. $1 \mathrm{H}$ NMR $\left(500 \mathrm{MHz}, \mathrm{CDCl}_{3}\right) \delta 8.15(\mathrm{~s}, 1 \mathrm{H}, \mathrm{OH}), 7.42-7.37(\mathrm{~m}, 4 \mathrm{H}, \mathrm{ArH}), 7.35-7.31(\mathrm{~m}, 1 \mathrm{H}, \mathrm{ArH})$, 7.30-7.28 (m, 1H, ArH), 7.27-7.24 (m, 2H, ArH), 2.41 (s, 3H, Me), 1.69 (s, 3H, Me). 13C NMR (101 $\left.\mathrm{MHz}_{\mathrm{CDCl}}\right) \delta 159.50,141.10,137.89,137.31,136.73,130.38,129.90,129.84,129.09,128.54$, $127.25,21.12,16.12$.

\section{Optimization of red-light-mediated dual $\mathrm{Pd} / n \mathrm{Pr}$-DMQA+-catalyzed $\mathrm{C}-\mathrm{H}$ arylation}

Using 1-([1,1'-biphenyl]-2-yl)pyrrolidin-2-one 4a and benzenediazonium tetrafluoroborate 5a as 
model substrates, $\mathrm{Pd}(\mathrm{OAc})_{2}$ as catalyst, 3 as $\mathrm{PC}, \mathrm{MeOH}$ as solvent, the reaction time was screened and the results are outlined in Table S2. Under red LED $(\lambda \max =640 \mathrm{~nm})$, the reaction proceeded smoothly to afford the desired product $\mathbf{6 a}$ in 95\% NMR yield after 8 hours (Table S2, entry 1). By decreasing the reaction time to $6 \mathrm{~h}, 4 \mathrm{~h}$ and $2 \mathrm{~h}$, the desired product $6 \mathbf{a}$ was obtained in 95\%, 95\% and $85 \%$ NMR yield, respectively (Table S2, entries 2-4). To test the background reactions, several control experiments have also been performed. In the absence of PC $\mathbf{3}$, red light or $\mathrm{Pd}(\mathrm{OAc}) 2$, significantly lower yields ( $\leq 25 \%$ ) of $\mathbf{6 a}$ was observed for all the conditions (Table S2, entries 5-9), which is in accordance with the reported photocatalytic mechanism for this reaction. 12

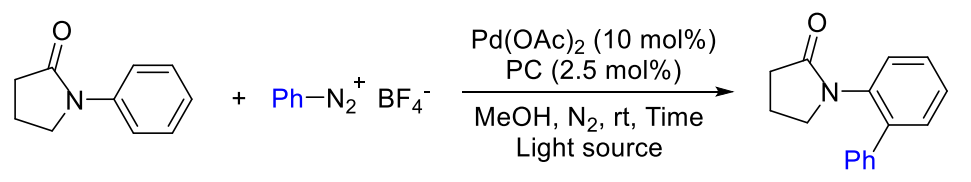

\begin{tabular}{|c|c|c|c|c|c|}
\hline \multicolumn{2}{|c|}{$4 a$} & \multicolumn{2}{|l|}{$5 a$} & \multicolumn{2}{|r|}{$6 a$} \\
\hline Entry ${ }^{a}$ & $\mathrm{PC}$ & Light source & Time $[\mathrm{h}]$ & Yield [\%] ${ }^{\mathrm{b}}$ & SM-4a $[\%]^{b}$ \\
\hline 1 & 3 & Red LED (640 nm) & 8 & 95 & trace \\
\hline 2 & 3 & Red LED $(640$ nm) & 6 & 95 & trace \\
\hline 3 & 3 & Red LED $(640$ nm) & 4 & 95 & trace \\
\hline 4 & 3 & Red LED $(640 \mathrm{~nm})$ & 2 & 85 & 10 \\
\hline 5 & - & Red LED (640 nm) & 4 & 22 & 73 \\
\hline 6 & - & Red LED $(640 \mathrm{~nm})$ & 8 & 25 & 70 \\
\hline 7 & 3 & - & 4 & 12 & 83 \\
\hline 8 & 3 & - & 8 & 16 & 79 \\
\hline $9^{c}$ & 3 & Red LED $(640 \mathrm{~nm})$ & 4 & trace & 95 \\
\hline \multicolumn{6}{|c|}{$\begin{array}{l}\text { The reaction was conducted with } 4 \mathrm{a}(0.1 \mathrm{mmol}), 5 \mathrm{a}(0.4 \mathrm{mmol}), \mathrm{Pd}(\mathrm{OAc})_{2}(10 \mathrm{~mol} \%) \\
\text { and } \mathrm{PC}(2.5 \mathrm{~mol} \%) \text { in } \mathrm{MeOH}(1.0 \mathrm{~mL}) .\end{array}$} \\
\hline
\end{tabular}

Table S2. Optimization and control experiments of red-light-mediated dual Pd/nPr-DMQA+catalyzed $\mathrm{C}\left(\mathrm{sp}_{2}\right)-\mathrm{H}$ arylation

\section{NMR results for control reactions for Table 1}

To further demonstrate the essential roles of red light, PC 3 and Pd catalyst, control experiments to determine the background reaction yields for all substrates have been conducted and the NMR results are presented in Table S3. In general, in the absence of red light, $\leq 15 \%$ NMR yield was observed for all the substrates, except for 36\% NMR yield for the reaction of 4a and 4chlorobenzenediazonium tetrafluoroborate $\mathbf{5 b}$. In the absence of $\mathbf{3}, \leq 40 \%$ NMR yield was observed 
for all the substrates, except for $45 \%$ NMR yield for the reaction of $\mathbf{4 a}$ and $\mathbf{5 b}$. However, in Sanford's work, the reaction of $\mathbf{4 a}$ and $\mathbf{5 b}$ was reported with $97 \%$ GC yield under 26 W CFL in the absence of $\mathrm{Ru}(\mathrm{bpy}) 32+$ for 4 hours.12 This major difference in reaction yield presumably results from the fact that diazonium salts show blue light absorption.13 The lower background observed in the present system highlights the great potential of low-energy red light towards reaction selectivity. At last, in the absence of Pd catalyst, trace amount of products 6 was observed for all the substrates, which supports the essential role of palladium for activating the substrate 4 during the catalytic cycle. 12

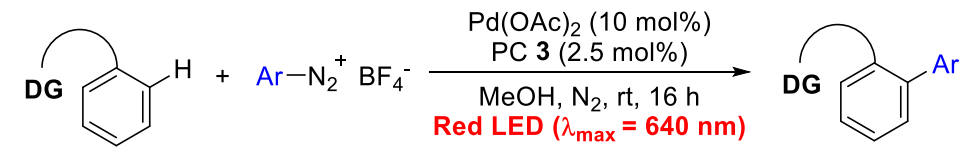

4

5

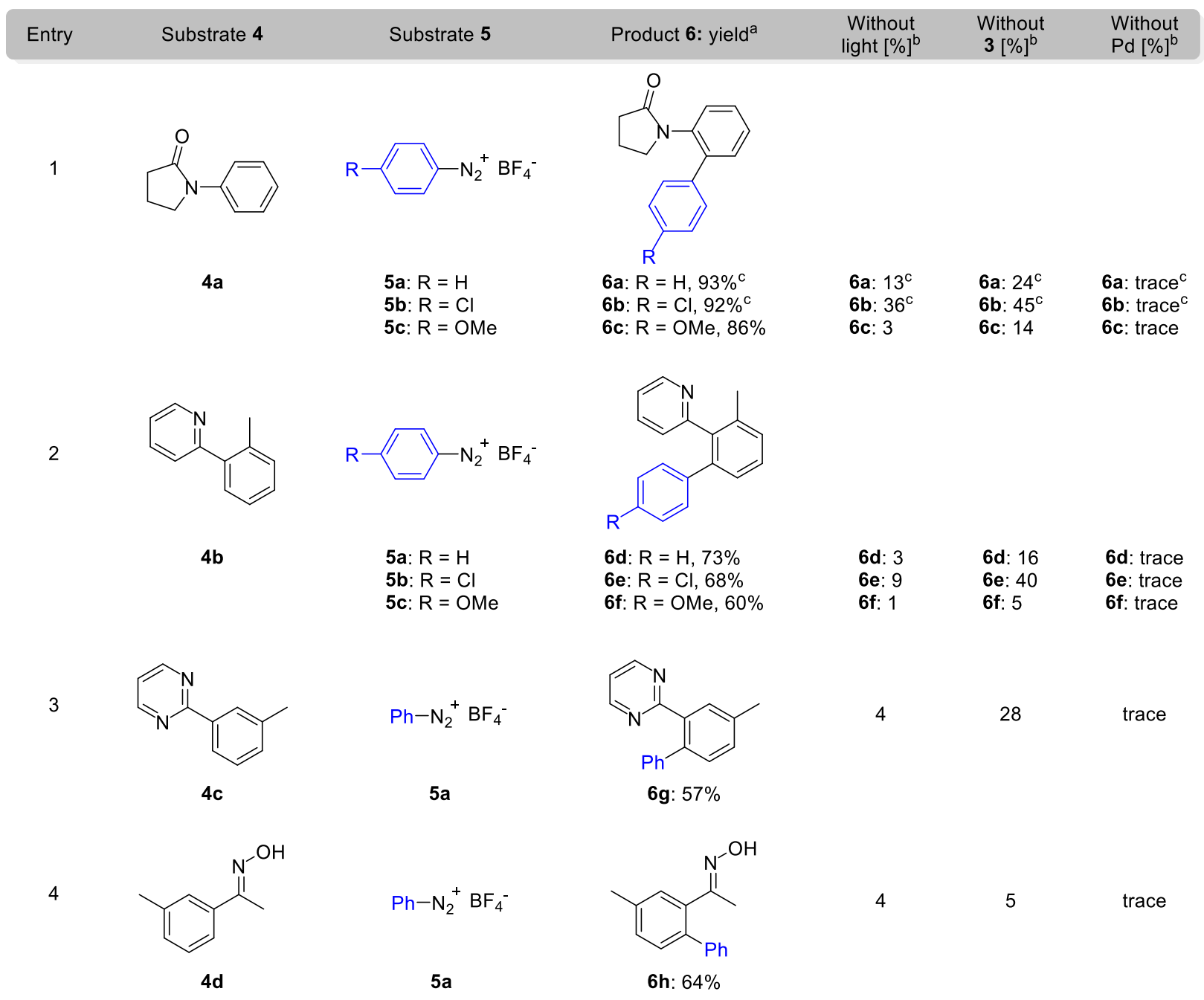

a The reaction was conducted with $4(0.2 \mathrm{mmol}), 5(0.8 \mathrm{mmol}), \mathrm{Pd}(\mathrm{OAc})_{2}(10 \mathrm{~mol} \%)$, and $3(2.5 \mathrm{~mol} \%)$ in $\mathrm{MeOH}(2.0 \mathrm{~mL})$. Isolated yields were shown. ${ }^{b}$ The reaction was conducted with $4(0.1 \mathrm{mmol}), 5(0.4 \mathrm{mmol}), \mathrm{Pd}(\mathrm{OAc})_{2}(10 \mathrm{~mol} \%)$, and $\mathbf{3}(2.5 \mathrm{~mol} \%)$ in $\mathrm{MeOH}(1.0 \mathrm{~mL})$. NMR yield by using 1,3,5-trimethoxybenzene as internal standard were shown. ${ }^{c}$ The reaction was ran for $4 \mathrm{~h}$.

Table S3. NMR results for control reactions for Table 1 
8. Typical procedure for ${ }_{n}$ Pr-DMQA+-catalyzed aerobic oxidative hydroxylation of arylboronic acids under red light

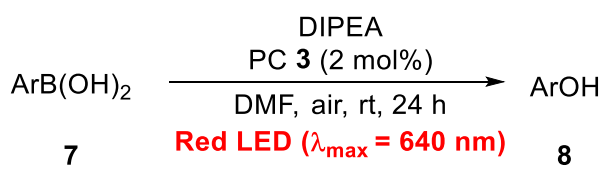

To a mixture of arylboronic acid 7 ( $0.50 \mathrm{mmol}, 1.0$ equiv.) and PC 3 (5.0 mg, $0.01 \mathrm{mmol}, 2 \mathrm{~mol} \%)$ in DMF (5.0 mL, 0.1 M) was added DIPEA (129 mg, $1.0 \mathrm{mmol}, 2.0$ equiv.) in a Schlenk tube. The solution was stirred at room temperature under red LED $\left(\lambda_{\max }=640 \mathrm{~nm}\right)$ irradiation in open to air (without bubbling air). After 24 hours, the reaction mixture was cooled to $0{ }^{\circ} \mathrm{C}$ and quenched by adding aqueous solution of $\mathrm{HCl}(10 \%, 5 \mathrm{~mL})$ slowly, followed by extracting with $\mathrm{Et}_{2} \mathrm{O}(3 \mathrm{x} 10 \mathrm{~mL})$. The combined organic layers were washed with brine $(10 \mathrm{~mL})$ and dried over anhydrous $\mathrm{Na}_{2} \mathrm{SO}_{4}$. After filtration, the solvent was removed under reduced pressure on RotaVap. The crude product was purified by flash chromatography $(F C)$ on silica gel (eluent: Hexanes/EtOAc $=20 / 1 \sim 1 / 2$ ) to furnish the desired product $\mathbf{8}$.

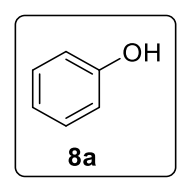

Phenol (8a): Yield (39 mg, 83\%). A colorless solid. $\mathrm{Rf}_{\mathrm{f}}=0.4($ Hexanes/EtOAc $=5 / 1)$. FC $($ Hexanes/EtOAc $=15 / 1) . \quad 1 \mathrm{H} \mathrm{NMR}\left(500 \mathrm{MHz}, \mathrm{CDCl}_{3}\right) \delta$ 7.27-7.23 (m, 2H, ArH), 6.96-6.92 (m, $1 \mathrm{H}, \mathrm{ArH}), 6.87-6.83$ (m, 2H, ArH), 5.10 (s, 1H, OH). ${ }_{13} \mathrm{C} \mathrm{NMR}\left(126 \mathrm{MHz}, \mathrm{CDCl}_{3}\right) \delta 155.75,129.89$, $120.97,115.50$.

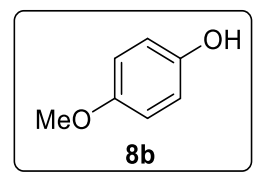

4-Methoxyphenol (8b): Yield (44 mg, 71\%). A colorless solid. $\mathrm{R}_{\mathrm{f}}=0.2($ Hexanes/EtOAc $=5 / 1)$. FC $($ Hexanes/EtOAc $=10 / 1) .1 \mathrm{H} \mathrm{NMR}\left(400 \mathrm{MHz}, \mathrm{CDCl}_{3}\right) \delta$ 6.84-6.77 (m, 4H, ArH), $4.76(\mathrm{~s}, 1 \mathrm{H}$, $\mathrm{OH}), 3.80$ (s, 3H, OMe). ${ }_{13} \mathrm{C}$ NMR $\left(101 \mathrm{MHz}, \mathrm{CDCl}_{3}\right) \delta 153.89,149.59,116.19,115.02,55.96$. 


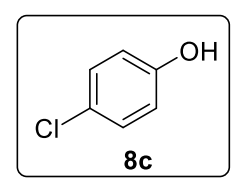

4-Chlorophenol (8c): Yield (52 mg, 81\%). A colorless oil. $\mathrm{Rf}_{\mathrm{f}}=0.4($ Hexanes/EtOAc $=5 / 1)$. FC $($ Hexanes/EtOAc $=10 / 1) .1 \mathrm{H} \mathrm{NMR}\left(400 \mathrm{MHz}, \mathrm{CDCl}_{3}\right) \delta$ 7.21-7.18 (m, 2H, ArH), 6.78-6.75 (m, $2 \mathrm{H}, \mathrm{ArH}), 4.86(\mathrm{~s}, 1 \mathrm{H}, \mathrm{OH}) .{ }_{13} \mathrm{C} \mathrm{NMR}\left(101 \mathrm{MHz}, \mathrm{CDCl}_{3}\right) \delta 154.22,129.67,125.83,116.80$.

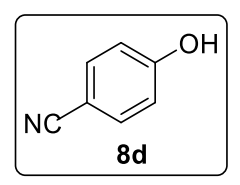

4-Cyanophenol (8d): Yield (52 mg, 87\%). A light yellow solid. $\mathrm{Rf}_{\mathrm{f}}=0.1($ Hexanes/EtOAc $=3 / 1)$. $\mathrm{FC}(\mathrm{Hexanes} / \mathrm{EtOAc}=5 / 1) .1 \mathrm{H} \mathrm{NMR}\left(400 \mathrm{MHz}, \mathrm{CDCl}_{3}\right) \delta$ 7.57-7.54 (m, 2H, ArH), 6.96-6.93 (m, 2H, ArH), 6.69 (s, 1H, OH). 13C NMR (101 MHz, CDCl 3 ) $\delta 160.34,134.47,119.37,116.61,103.16$.

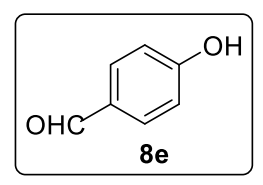

4-Hydroxybenzaldehyde (8e): Yield (40 mg, 66\%). A white solid. $\mathrm{R}_{\mathrm{f}}=0.3($ Hexanes/EtOAc $=3 / 1)$. FC (Hexanes/EtOAc = 5/1). 1H NMR (400 MHz, Methanol- $d 4) \delta 9.76(\mathrm{~s}, 1 \mathrm{H}, \mathrm{CHO}), 7.78-7.75(\mathrm{~m}$, 2H, ArH), 6.93-6.90 (m, 2H, ArH). 13C NMR (101 MHz, Methanol-d4) $\delta$ 192.81, 165.15, 133.42, $130.30,116.85$.

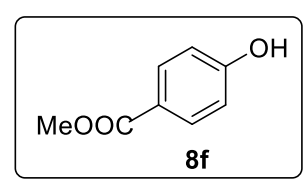

Methyl 4-hydroxybenzoate (8f): Yield (61 mg, 80\%). A white solid. $\mathrm{Rf}_{\mathrm{f}}=0.3($ Hexanes $/$ EtOAc $=$ 3/1). FC (Hexanes/EtOAc = 5/1). 1 H NMR (400 MHz, CDCl 3 ) $\delta$ 7.97-7.93 (m, 2H, ArH), 6.91-6.87 (m, 2H, ArH), 6.46 (s, 1H, OH), 3.90 (s, 3H, OMe). $\left.{ }_{13} \mathrm{C} \mathrm{NMR} \mathrm{(101} \mathrm{MHz,} \mathrm{CDCl}_{3}\right) \delta 167.65,160.44$, $132.11,122.39,115.45,52.26$.

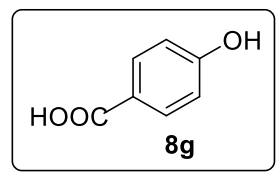

4-Hydroxybenzoic acid (8g): Yield (28 mg, 41\%). A white solid. $\mathrm{R}_{\mathrm{f}}=0.2($ Hexanes/EtOAc $=1 / 1)$. FC $($ Hexanes/EtOAc = 1/1). $1 \mathrm{H}$ NMR $(400 \mathrm{MHz}$, Methanol- $d 4) \delta$ 7.89-7.86 (m, 2H, ArH), 6.83-6.80 $(\mathrm{m}, 2 \mathrm{H}, \mathrm{ArH}) .{ }_{13 \mathrm{C}} \mathrm{NMR}\left(126 \mathrm{MHz}\right.$, Methanol- $\left.d_{4}\right) \delta 170.26,163.54,133.13,122.81,116.13$. 


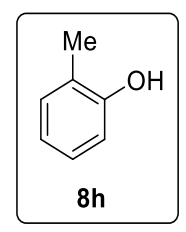

$o$-Cresol (8h): Yield (39 mg, 72\%). A light yellow oil. $\mathrm{Rf}_{\mathrm{f}}=0.5($ Hexanes $/$ EtOAc $=5 / 1) . \mathrm{FC}$ $(\mathrm{Hexanes} / \mathrm{EtOAc}=20 / 1) .1 \mathrm{H} \mathrm{NMR}\left(500 \mathrm{MHz}, \mathrm{CDCl}_{3}\right) \delta$ 7.14-7.11 (m, 1H, ArH), 7.11-7.06 (m, 1H, ArH), 7.14-7.11 (m, 1H, ArH), 6.77 (dd, $J=8.0,1.5 \mathrm{~Hz}, 1 \mathrm{H}, \mathrm{ArH}), 4.65$ (s, 1H, OH), 2.26 (s, 3H, Me). ${ }_{13} \mathrm{C} \mathrm{NMR}\left(101 \mathrm{MHz}, \mathrm{CDCl}_{3}\right) \delta 153.89,131.16,127.27,123.80,120.89,115.01,15.84$.

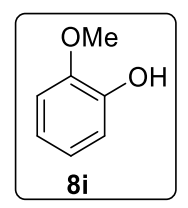

2-Methoxyphenol (8i): Yield (34 mg, 55\%). A white solid. $\mathrm{Rf}_{\mathrm{f}}=0.5($ Hexanes $/$ EtOAc $=5 / 1)$. FC $(\mathrm{Hexanes} / \mathrm{EtOAc}=20 / 1) .{ }_{1} \mathrm{H} \mathrm{NMR}\left(500 \mathrm{MHz}, \mathrm{CDCl}_{3}\right) \delta 6.94-6.91(\mathrm{~m}, 1 \mathrm{H}, \mathrm{ArH}), 6.90-6.84(\mathrm{~m}, 3 \mathrm{H}$, $\mathrm{ArH}), 5.60$ (s, 1H, OH), 3.89 (s, 3H, OMe). 13C NMR (126 MHz, CDCl 3$) \delta 146.82,145.92,121.66$, $120.34,114.72,110.90,55.97$.

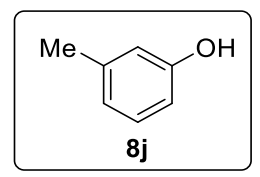

$m$-Cresol (8j): Yield (36 mg, 67\%). A colorless oil. $\mathrm{Rf}_{\mathrm{f}}=0.5($ Hexanes $/$ EtOAc $=5 / 1)$. FC $($ Hexanes/EtOAc $=20 / 1) .1{ }_{1} \mathrm{NMR}\left(400 \mathrm{MHz}, \mathrm{CDCl}_{3}\right) \delta 7.13(\mathrm{dd}, J=7.6,8.0 \mathrm{~Hz}, 1 \mathrm{H}, \mathrm{ArH}), 6.76$ $(\mathrm{d}, J=7.6 \mathrm{~Hz}, 1 \mathrm{H}, \mathrm{ArH}), 6.68-6.62(\mathrm{~m}, 2 \mathrm{H}, \mathrm{ArH}), 4.76(\mathrm{~s}, 1 \mathrm{H}, \mathrm{OH}), 2.32$ (s, 3H, Me). ${ }_{13 \mathrm{C}} \mathrm{NMR}$ $\left(101 \mathrm{MHz}, \mathrm{CDCl}_{3}\right) \delta 155.53,139.97,129.56,121.77,116.15,112.40,21.48$.

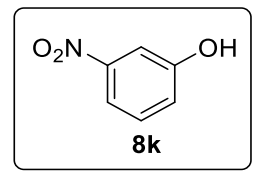

3-Nitrophenol (8k): Yield (51 mg, 73\%). A white solid. $\mathrm{R}_{\mathrm{f}}=0.4($ Hexanes/EtOAc $=3 / 1)$. FC $($ Hexanes/EtOAc $=10 / 1) .1 \mathrm{H} \mathrm{NMR}\left(400 \mathrm{MHz}, \mathrm{CDCl}_{3}\right) \delta 7.81(\mathrm{dd}, J=8.4,2.0 \mathrm{~Hz}, 1 \mathrm{H}, \mathrm{ArH}), 7.71$ $(\mathrm{dd}, J=2.4,2.0 \mathrm{~Hz}, 1 \mathrm{H}, \mathrm{ArH}), 7.41$ (dd, $J=8.4,8.4 \mathrm{~Hz}, 1 \mathrm{H}, \mathrm{ArH}), 7.19$ (dd, $J=8.4,2.4 \mathrm{~Hz}, 1 \mathrm{H}$, $\mathrm{ArH}), 5.63(\mathrm{~s}, 1 \mathrm{H}, \mathrm{OH}) .{ }_{13} \mathrm{C} \mathrm{NMR}\left(101 \mathrm{MHz}, \mathrm{CDCl}_{3}\right) \delta 156.39,149.25,130.46,122.16,116.08$, 110.70 .

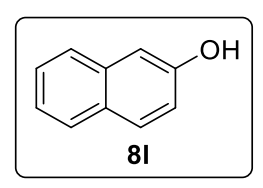

Naphthalen-2-ol (8I): Yield (47 mg, 65\%). A light yellow solid. $\mathrm{R}_{\mathrm{f}}=0.3($ Hexanes/EtOAc $=5 / 1)$. FC $($ Hexanes/EtOAc $=15 / 1) .{ }_{1} \mathrm{H} \mathrm{NMR}\left(400 \mathrm{MHz}, \mathrm{CDCl}_{3}\right) \delta 7.78(\mathrm{dd}, J=8.4,8.4 \mathrm{~Hz}, 2 \mathrm{H}, \mathrm{ArH})$, $7.69(\mathrm{~d}, J=8.4 \mathrm{~Hz}, 1 \mathrm{H}, \mathrm{ArH}), 7.48-7.43$ (m, 1H, ArH), 7.38-7.33 (m, 1H, ArH), 7.16 (d, $J=2.4 \mathrm{~Hz}$, 
$1 \mathrm{H}, \mathrm{ArH}), 7.12(\mathrm{dd}, J=8.8,2.4 \mathrm{~Hz}, 1 \mathrm{H}, \mathrm{ArH}), 5.16(\mathrm{~s}, 1 \mathrm{H}, \mathrm{OH}), 13 \mathrm{C} \mathrm{NMR}\left(101 \mathrm{MHz}, \mathrm{CDCl}_{3}\right) \delta$ $153.36,134.70,130.01,129.09,127.90,126.68,126.51,123.79,117.84,109.67$.

\section{Optimization of $n$ Pr-DMQA+-catalyzed aerobic oxidative hydroxylation}

Using phenylboronic acid 7a as model substrate, DIPEA as base, $\mathbf{3}$ as PC, air as oxidant, DMF as solvent, the reaction time was screened and the results are shown in Table S4. Under red LED ( $\lambda_{\max }$ $=640 \mathrm{~nm}$ ), the reaction proceeded smoothly to afford phenol 8a in 77\% NMR yield after 18 hours (Table S4, entry 1). By increasing the reaction time to 24 hours, $8 \mathbf{a}$ was obtained in $87 \%$ NMR yield, along with $83 \%$ isolated yield (Table S4, entry 2). Running the reaction for $28 \mathrm{~h}$ did not improve the result (Table S4, entry 3). Several control experiments have also been performed. In the absence of 3 or red light, little or no conversion was observed (Table S4, entries 4-5), which is consistent with the photocatalytic model for this reaction in the reported literature.14-17

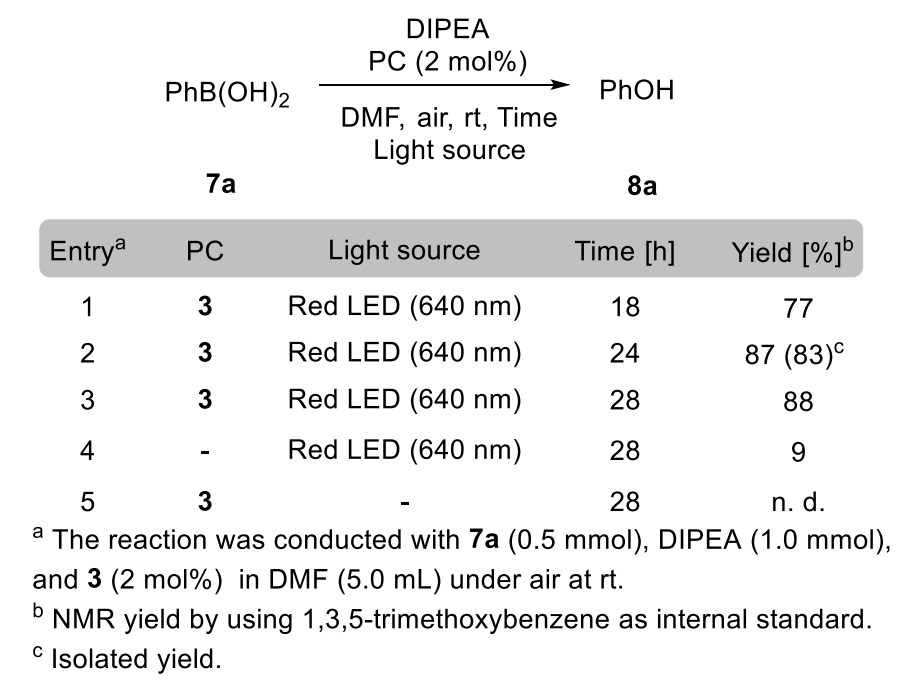

Table S4. Optimization and control experiments of ${ }_{n} \operatorname{Pr}-\mathrm{DMQA}+$-catalyzed aerobic oxidative hydroxylation of arylboronic acids

\section{Experimental procedure for red-light-induced ${ }_{n}$ Pr-DMQA+-catalyzed oxygenation}

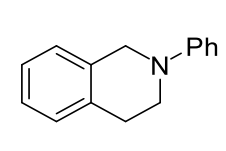

9

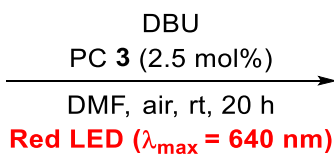

$\operatorname{Red} \operatorname{LED}\left(\lambda_{\max }=640 \mathrm{~nm}\right)$

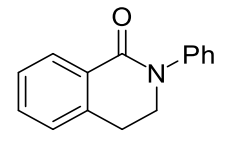

$10,92 \%$

To a mixture of tertiary amine 9 (42 mg, $0.20 \mathrm{mmol}, 1.0$ equiv.) and PC 3 ( $2.5 \mathrm{mg}, 0.005 \mathrm{mmol}, 2.5$ 
mol\%) in DMF (2.0 mL, 0.1 M) was added DBU (46 mg, $0.3 \mathrm{mmol}, 1.5$ equiv.) in a Schlenk tube. The solution was stirred at room temperature under red LED $\left(\lambda_{\max }=640 \mathrm{~nm}\right)$ irradiation in open to air (without bubbling air) for 20 hours. The mixture was concentrated in vacuo to yield the crude product, which was purified by flash chromatography $(\mathrm{FC})$ on silica gel (eluent: Hexanes/EtOAc $=$ 10/1 5/1) to yield the desired amide 10 as a white solid.

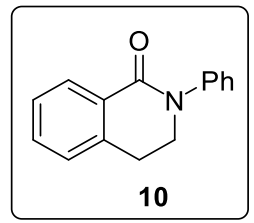

2-phenyl-3,4-dihydroisoquinolin-1(2H)-one (10): Yield (41 $\mathrm{mg}, 92 \%)$. A white solid. $\mathrm{R}_{\mathrm{f}}=0.3$ $($ Hexanes/EtOAc $=3 / 1) . \mathrm{FC}($ Hexanes/EtOAc $=10 / 1 \sim 5 / 1) .1 \mathrm{H} \mathrm{NMR}\left(400 \mathrm{MHz}, \mathrm{CDCl}_{3}\right) \delta 8.17(\mathrm{~d}$, $J=7.6 \mathrm{~Hz}, 1 \mathrm{H}, \mathrm{ArH}), 7.55-7.36(\mathrm{~m}, 6 \mathrm{H}, \mathrm{ArH}), 7.29-7.24(\mathrm{~m}, 2 \mathrm{H}, \mathrm{ArH}), 4.01(\mathrm{t}, J=6.4 \mathrm{~Hz}, 2 \mathrm{H}$, $\left.\mathrm{CH}_{2}\right), 3.16$ (t, $\left.J=6.4 \mathrm{~Hz}, 2 \mathrm{H}, \mathrm{CH}_{2}\right) .13 \mathrm{C} \mathrm{NMR}\left(101 \mathrm{MHz}, \mathrm{CDCl}_{3}\right) \delta 164.29,143.24,138.42,132.13$, $129.85,129.02,128.86,127.30,127.05,126.34,125.43,49.53,28.75$.

\section{Experimental procedure for red-light-induced ${ }_{n}$ Pr-DMQA+-catalyzed ATRA}

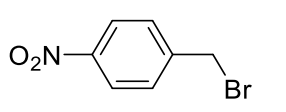

11

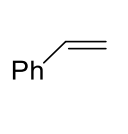

12

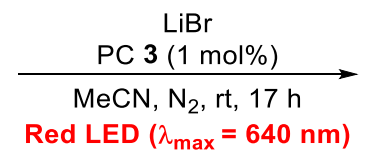

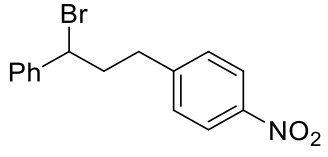

$13,59 \%$

In a N2 glove box, 3 (1.0 mg, $0.002 \mathrm{mmol}, 1 \mathrm{~mol} \%$ ), 4-nitrobenzyl bromide 11 (43 mg, $0.2 \mathrm{mmol}$, 1.0 equiv.) and $\mathrm{LiBr}$ (35 mg, $0.4 \mathrm{mmol}, 2.0$ equiv.) were added to an oven-dried (overnight) Schlenk tube containing a stirring bar, followed by adding dry $\mathrm{MeCN}(1.0 \mathrm{~mL}, 0.2 \mathrm{M})$ and styrene 12 (208 $\mathrm{mg}, 2.0 \mathrm{mmol}$, 10.0 equiv.). The Schlenk tube was then sealed, removed from the glove box and stirred at room temperature under red $\operatorname{LED}\left(\lambda_{\max }=640 \mathrm{~nm}\right)$ irradiation for 17 hours. The mixture was concentrated in vacuo to yield the crude product, which was purified by flash chromatography $(\mathrm{FC})$ on silica gel (eluent: Hexanes/EtOAc $=200 / 1)$ to yield the desired product $\mathbf{1 3}$ as a colorless oil. 


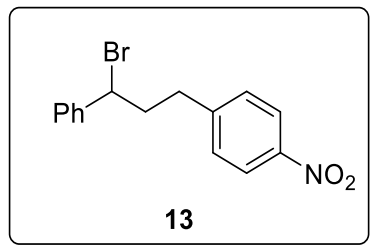

1-(3-bromo-3-phenylpropyl)-4-nitrobenzene (13): Yield (38 mg, 59\%). A colorless oil. $\mathrm{R}_{\mathrm{f}}=0.4$ $($ Hexanes/EtOAc $=10 / 1) . F C(H e x a n e s / E t O A c=200 / 1) .1 \mathrm{H} \mathrm{NMR}\left(400 \mathrm{MHz}, \mathrm{CDCl}_{3}\right) \delta 8.18-8.14$ (m, 2H, ArH), 7.40-7.28 (m, 7H, ArH), $4.88(\mathrm{dd}, J=8.4,6.0 \mathrm{~Hz}, 1 \mathrm{H}, \mathrm{CH}), 2.95$ (ddd, $J=14.4,9.2$, $5.6 \mathrm{~Hz}, 1 \mathrm{H}, \mathrm{CH}_{2}$ ), 2.82 (ddd, $J=14.4,8.8,6.4 \mathrm{~Hz}, 1 \mathrm{H}, \mathrm{CH}_{2}$ ), 2.69-2.59 (m, 1H, $\mathrm{CH}_{2}$ ), 2.50-2.41 (m, $1 \mathrm{H}, \mathrm{CH}_{2}$ ). ${ }_{13} \mathrm{C} \mathrm{NMR}\left(101 \mathrm{MHz}, \mathrm{CDCl}_{3}\right) \delta 148.37,146.77,141.53,129.47,128.99,128.78,127.34$, $123.94,54.09,40.90,34.30$.

\section{Optimization of red-light-induced $n$ Pr-DMQA+-catalyzed intermolecular ATRA}

Initial examination of ATRA reaction was tested by reacting 4-nitrobenzyl bromide 11 (1.0 equiv.) with styrene 12 (5.0 equiv.) in the presence of $\mathrm{LiBr}$ (2.0 equiv.) and PC 3 in MeCN under red LED $\left(\lambda_{\max }=640 \mathrm{~nm}\right)($ Table S5). Running the reaction with $1 \mathrm{~mol} \%$ of 3 at $\mathrm{rt}$ for 20 hours delivered the desired adduct 13 in 40\% NMR yield, along with 50\% starting material 11 (SM-11) (Table S5, entry 1). Increasing the PC loading of $\mathbf{3}$ to $2.5 \mathrm{~mol} \%$ gave lower yield of $\mathbf{1 3}$, along with more byproducts, which could be the dimer, polymers or other side-products from the radical intermediates (Table S5, entry 2). Thus, $1 \mathrm{~mol} \%$ the PC loading was chosen for all the follow-up screenings. When the reaction was run at $50{ }^{\circ} \mathrm{C}$ for 24 hours, only $25 \%$ of desired product 13 was observed, along with a lot of byproducts and no SM-11 (Table S5, entry 3). Two control experiments have also been performed, and no reaction occurred at $50{ }^{\circ} \mathrm{C}$ in the absence of red light or PC 3 (Table S5, entries 4-5). Performing the reaction at $35^{\circ} \mathrm{C}$ didn ${ }^{`}$ improve any reaction result (Table S5, entry 6). When the reaction was conducted in a higher concentration $(0.2 \mathrm{M})$, the reaction yield was improved from $40 \%$ to $54 \%$ (Table S5, entry 1 vs entry 7). Moreover, when the reaction was performed with 10.0 equiv. of styrene 12 in a higher concentration $(0.2 \mathrm{M}), 13$ was achieved in $65 \%$ yield, along with 12\% SM-11 (Table S5, entry 8). Further increasing the reaction concentration $(0.4 \mathrm{M})$ did not increase the reaction yield (Table S5, entry 9). Thus, the current optimal reaction condition is reacting 11 (1.0 equiv.) with styrene 12 (10.0 equiv.) in the presence of $\operatorname{LiBr}$ (2.0 equiv.) and $\mathrm{PC} 3$ $(1 \mathrm{~mol} \%)$ in $\mathrm{MeCN}(0.2 \mathrm{M})$ at $\mathrm{rt}$ for 17 hours under red LED $\left(\lambda_{\max }=640 \mathrm{~nm}\right)($ Table S5, entry 8$)$. 


\begin{tabular}{|c|c|c|c|c|c|c|c|}
\hline \multirow[b]{3}{*}{ Entry ${ }^{a}$} & \multirow{2}{*}{\multicolumn{2}{|c|}{$\mathrm{O}_{2} \mathrm{~N}-$}} & \multirow{2}{*}{$\begin{array}{l}\mathrm{Ph} \\
12\end{array}$} & $\frac{\mathrm{LiBr}}{\mathrm{PC}(\mathrm{x} \mathrm{mol} \%)} \underset{\substack{\text { MeCN, } \mathrm{N}_{2}, \text { Temp., Time } \\
\text { Light source }}}{\longrightarrow}$ & \multirow[t]{2}{*}{$\gamma^{\mathrm{Br}}$} & \multirow[t]{2}{*}{$\mathrm{NO}_{3}$} & \multirow[b]{3}{*}{ SM-11 $[\%]^{b}$} \\
\hline & & & & & & & \\
\hline & $\mathrm{PC}$ & PC loading/x & Light source & Temp. $\left[{ }^{\circ} \mathrm{C}\right]$ & Time $[\mathrm{h}]$ & Yield [\%] ${ }^{\mathrm{b}}$ & \\
\hline 1 & 3 & 1 & Red LED (640 nm) & $\mathrm{rt}$ & 20 & 40 & 50 \\
\hline 2 & 3 & 2.5 & Red LED (640 nm) & $\mathrm{rt}$ & 20 & 30 & 50 \\
\hline 3 & 3 & 1 & Red LED $(640 \mathrm{~nm})$ & 50 & 24 & 25 & 0 \\
\hline 4 & 3 & 1 & - & 50 & 24 & 0 & 95 \\
\hline 5 & - & 1 & Red LED $(640 \mathrm{~nm})$ & 50 & 24 & 0 & 95 \\
\hline 6 & 3 & 1 & Red LED (640 nm) & 35 & 18 & 42 & 20 \\
\hline $7^{c}$ & 3 & 1 & Red LED (640 nm) & $\mathrm{rt}$ & 20 & 54 & 30 \\
\hline $8^{d}$ & 3 & 1 & Red LED $(640 \mathrm{~nm})$ & $\mathrm{rt}$ & 17 & 65 & 12 \\
\hline $9^{e}$ & 3 & 1 & Red LED (640 nm) & $\mathrm{rt}$ & 17 & 47 & 13 \\
\hline \multicolumn{8}{|c|}{ a The reaction was ran with $11(0.2 \mathrm{mmol}), 12(1.0 \mathrm{mmol}), \mathrm{PC}(\mathrm{x} \mathrm{mol} \%)$ and $\mathrm{LiBr}(0.4 \mathrm{mmol}) \mathrm{in} \mathrm{MeCN}(2 \mathrm{~mL})$. } \\
\hline \multicolumn{8}{|c|}{ b NMR yield by using 1,3,5-trimethoxybenzene as internal standard. } \\
\hline \multicolumn{8}{|c|}{ c $1 \mathrm{~mL}$ of $\mathrm{MeCN}$ was used. } \\
\hline \multicolumn{8}{|c|}{ d $2.0 \mathrm{mmol}$ of 12 and $1 \mathrm{~mL}$ of $\mathrm{MeCN}$ were used. } \\
\hline \multicolumn{8}{|c|}{ e $2.0 \mathrm{mmol}$ of 12 and $0.5 \mathrm{~mL}$ of $\mathrm{MeCN}$ were used. } \\
\hline
\end{tabular}

Table S5. Initial examination of red-light-induced ${ }_{n} \operatorname{Pr}-\mathrm{DMQA}+-$ catalyzed intermolecular ATRA

Based on the above preliminary results, further optimization by screening the solvents and reaction times were carried out and the results are summarized in Table S6. The examination of solvent effects revealed that the reaction in $\mathrm{MeCN}$ provided a higher yield than those in $\mathrm{DMF} / \mathrm{H}_{2} \mathrm{O}$ (1/4),18,19 MeOH and DMSO (Table S6, entry 1 vs 2-4). Increasing the reaction time from 17 to 24 hours in MeCN gave the product $\mathbf{1 3}$ in 59\% yield along with trace amount of SM-11 (Table S6, entry 5). Next, several control experiments have also been conducted. No reaction occurred in the absence of $\mathbf{3}$ or red light, which is consistent with the results at $50{ }^{\circ} \mathrm{C}$ (Table S6, entries 6-7 vs Table S5, entries 4-5). In addition, in the absence of $\mathrm{LiBr}, 13$ was obtained in $14 \%$ yield along with $75 \%$ of SM-11 (Table S6, entry 8). On the basis of these experiments, we chose reacting 11 (1.0 equiv.) with styrene 12 (10.0 equiv.) in the presence of $\mathrm{LiBr}$ (2.0 equiv.) and $\mathrm{PC} 3$ ( $1 \mathrm{~mol} \%)$ in $\mathrm{MeCN}(0.2$ M) at $\mathrm{rt}$ for 17 hours under red LED $\left(\lambda_{\max }=640 \mathrm{~nm}\right)$ as the optimal reaction condition (Table S6, entry 1). 


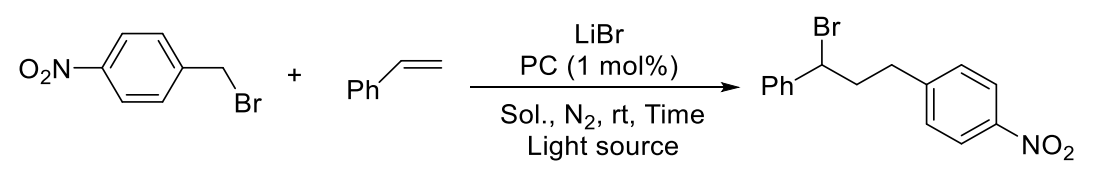

$11 \quad 12 \quad 13$

\begin{tabular}{|c|c|c|c|c|c|c|}
\hline Entry $^{a}$ & PC & Light source & Solvent & Time $[\mathrm{h}]$ & Yield $[\%]^{\mathrm{b}}$ & SM-11 $[\%]^{\mathrm{b}}$ \\
\hline 1 & 3 & Red LED $(640 \mathrm{~nm})$ & $\mathrm{MeCN}$ & 17 & $65(59)^{c}$ & 12 \\
\hline 2 & 3 & Red LED $(640 \mathrm{~nm})$ & $\mathrm{DMF} / \mathrm{H}_{2} \mathrm{O}(1 / 4)$ & 17 & messy & 0 \\
\hline 3 & 3 & Red LED $(640 \mathrm{~nm})$ & $\mathrm{MeOH}$ & 17 & 0 & 95 \\
\hline 4 & 3 & Red LED $(640 \mathrm{~nm})$ & DMSO & 17 & 30 & trace \\
\hline 5 & 3 & Red LED $(640 \mathrm{~nm})$ & $\mathrm{MeCN}$ & 24 & 59 & trace \\
\hline 6 & - & Red LED $(640 \mathrm{~nm})$ & $\mathrm{MeCN}$ & 17 & 0 & 95 \\
\hline 7 & 3 & - & $\mathrm{MeCN}$ & 17 & 0 & 95 \\
\hline $8^{d}$ & 3 & Red LED $(640 \mathrm{~nm})$ & $\mathrm{MeCN}$ & 17 & 14 & 75 \\
\hline
\end{tabular}

Table S6. Further optimization of red-light-induced ${ }_{n} \mathrm{Pr}-\mathrm{DMQA}+-$ catalyzed intermolecular ATRA

\section{Proposed mechanism for red-light-induced ${ }_{n} \operatorname{Pr}-\mathrm{DMQA}+$-catalyzed intermolecular ATRA}

Based on previous work, $18-22$ plausible reaction mechanism was proposed for red-light-induced ${ }_{n} \operatorname{Pr}-$ DMQA+-catalyzed intermolecular ATRA (Scheme S1). Firstly, oxidative quenching of the red lightinduced excited state of ${ }_{n} \operatorname{Pr}-\mathrm{DMQA}+*$ by 4-nitrobenzyl bromide 11 generates a 4-nitrobenzyl radical $\left(4-\mathrm{NO}_{2}-\mathrm{PhCH}_{2} \mathrm{Br} / 4-\mathrm{NO}_{2}-\mathrm{PhCH}_{2} \cdot=-0.65 \mathrm{~V}\right.$ vs. SCE),23,24 along with bromide anion. Then, addition of the 4-nitrobenzyl radical to styrene $\mathbf{1 2}$ forms another benzyl radical intermediate $\mathbf{S 3}$, which undergoes a single electron transfer (SET) process with ${ }_{n} \mathrm{Pr}-\mathrm{DMQA} \cdot++$ to regenerate the $\mathrm{PC}{ }_{n} \mathrm{Pr}-$ $\mathrm{DMQA}+$ and form the carbocation species $\mathbf{S 4}\left(\mathrm{PhCH}+\mathrm{CH}_{3} / \mathrm{PhCH} \cdot \mathrm{CH}_{3}=+0.37 \mathrm{~V}\right.$ vs. SCE $) .22,25$ Final product $\mathbf{1 3}$ is formed by the nucleophilic attack of bromide anion to the carbocation species $\mathbf{S 4}$. Alternatively, $\mathbf{1 3}$ could be also obtained through a radical chain transfer mechanism. 


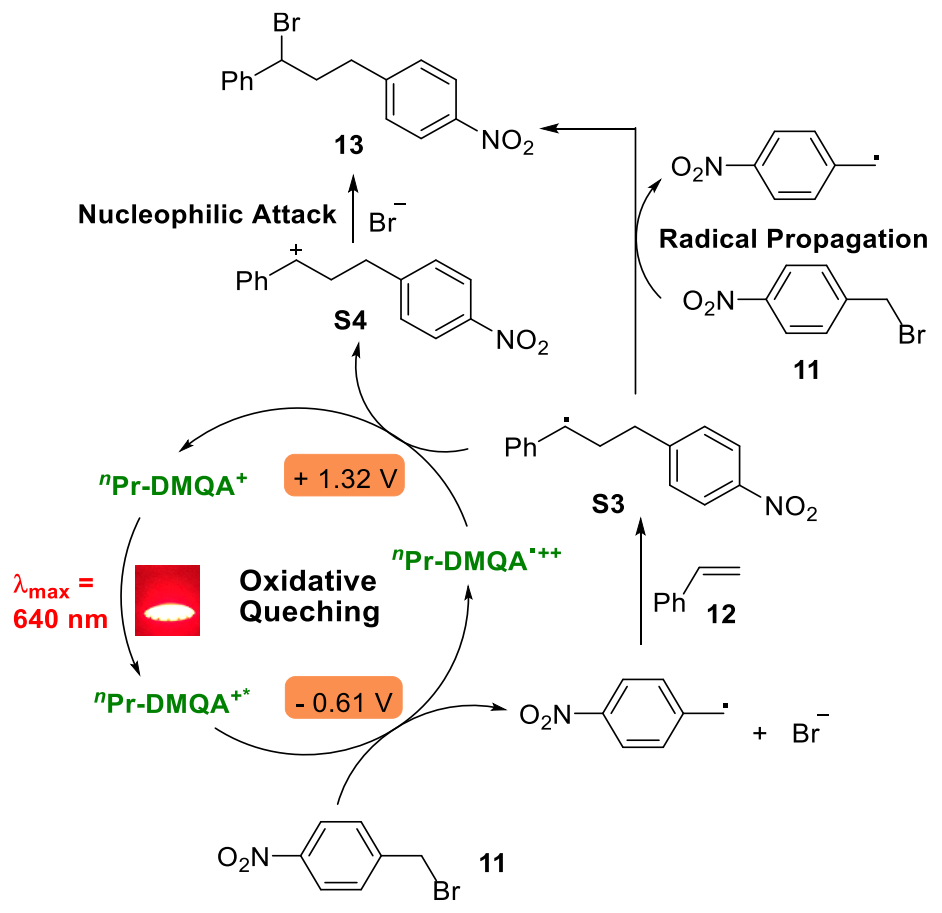

Scheme S1. Proposed mechanism for red-light-induced ${ }_{n}$ Pr-DMQA+-catalyzed ATRA

\section{Experimental procedure for red-light-mediated dual Au/nPr-DMQA+-catalyzed C(sp)-H arylation}

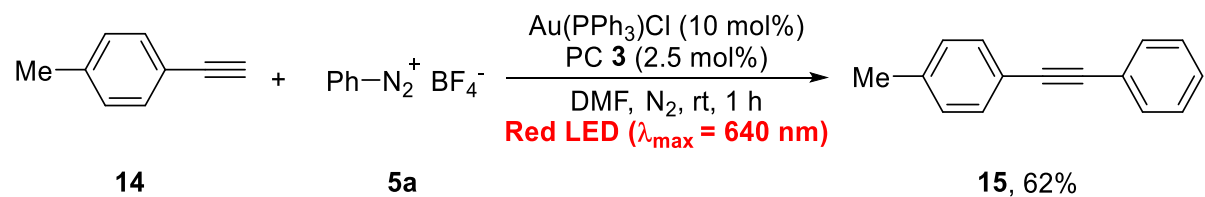

In a $\mathrm{N}_{2}$ glove box, $\mathrm{Au}\left(\mathrm{PPh}_{3}\right) \mathrm{Cl}(9.9 \mathrm{mg}, 0.02 \mathrm{mmol}, 10 \mathrm{~mol} \%), 3$ (2.5 mg, $\left.0.005 \mathrm{mmol}, 2.5 \mathrm{~mol} \%\right)$, 1-ethynyl-4-methylbenzene 14 (23 mg, $0.2 \mathrm{mmol}, 1.0$ equiv.) and benzenediazonium tetrafluoroborate 5a (154 mg, $0.8 \mathrm{mmol}, 4.0$ equiv.) were added to an oven-dried (overnight) Schlenk tube containing a stirring bar, followed by adding dry DMF $(2.0 \mathrm{~mL}, 0.1 \mathrm{M})$. The Schlenk tube was then sealed, removed from the glove box and stirred at room temperature under red LED $\left(\lambda_{\max }=640 \mathrm{~nm}\right)$ irradiation. After 1 hour, the mixture was quenched with a saturated solution of $\mathrm{NaHCO}_{3}(2 \mathrm{~mL})$, followed by adding deionized water $(2 \mathrm{~mL})$. The crude reaction mixture was then extracted with ethyl acetate $(3 \times 10 \mathrm{~mL})$, and the combined organic layers were washed with brine $(20 \mathrm{~mL})$ and dried over anhydrous $\mathrm{Na}_{2} \mathrm{SO}_{4}$. After filtration, the solvent was removed under reduced pressure on RotaVap. The crude product was purified by flash chromatography (FC) on silica gel 
(eluent: Hexanes/EtOAc $=$ Hexanes $\sim 200 / 1$ ) to yield the desired product $\mathbf{1 5}$ as a white solid.

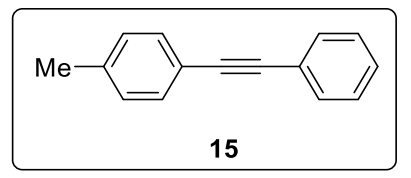

1-methyl-4-(phenylethynyl)benzene (15): Yield (24 mg, 62\%). A white solid. $\mathrm{Rf}_{\mathrm{f}}=0.45$ (Hexanes). FC $\left(\right.$ Hexanes/EtOAc $=$ Hexanes 200/1). ${ }_{1} \mathrm{H} \mathrm{NMR}\left(400 \mathrm{MHz}, \mathrm{CDCl}_{3}\right) \delta$ 7.56-7.53 (m, 2H, ArH), 7.45 (d, $J=7.6 \mathrm{~Hz}, 2 \mathrm{H}, \mathrm{ArH}), 7.38-7.31(\mathrm{~m}, 3 \mathrm{H}, \mathrm{ArH}), 7.17$ (d, $J=7.6 \mathrm{~Hz}, 2 \mathrm{H}, \mathrm{ArH}), 2.39$ (s, 3H). ${ }_{13} \mathrm{C}$ NMR $\left(101 \mathrm{MHz}, \mathrm{CDCl}_{3}\right) \delta 138.51,131.68,131.63,129.25,128.45,128.20,123.63,120.33$, $89.70,88.86,21.65$.

\section{Optimization of red-light-mediated dual Au/nPr-DMQA+-catalyzed C(sp)-H arylation}

Optimization of $\mathrm{C}(\mathrm{sp})-\mathrm{H}$ arylation by screening the solvents were performed and the results are presented in Table S7. The examination of solvent effects showed that the reaction in DMF gave a higher yield than those in DMSO and $\mathrm{MeOH}$ (Table S7, entry 1 vs 2-3). Control experiments have also been conducted. In the absence of 3 or red light, the desired product 15 was obtained in $24 \%$ and 5\% yield, respectively, while the reaction was messy in the absence of $\mathrm{Au}\left(\mathrm{PPh}_{3}\right) \mathrm{Cl}$ (Table $\mathrm{S} 7$, entries 4-6). Thus, we chose reacting 14 (1.0 equiv.) with benzenediazonium tetrafluoroborate 5a (4.0 equiv.) in the presence of $\mathrm{Au}\left(\mathrm{PPh}_{3}\right) \mathrm{Cl}(10 \mathrm{~mol} \%)$ and $\mathrm{PC} 3(2.5 \mathrm{~mol} \%)$ in DMF at rt for 1 hour under red LED $\left(\lambda_{\max }=640 \mathrm{~nm}\right)$ as the optimal reaction condition (Table S7, entry 1).

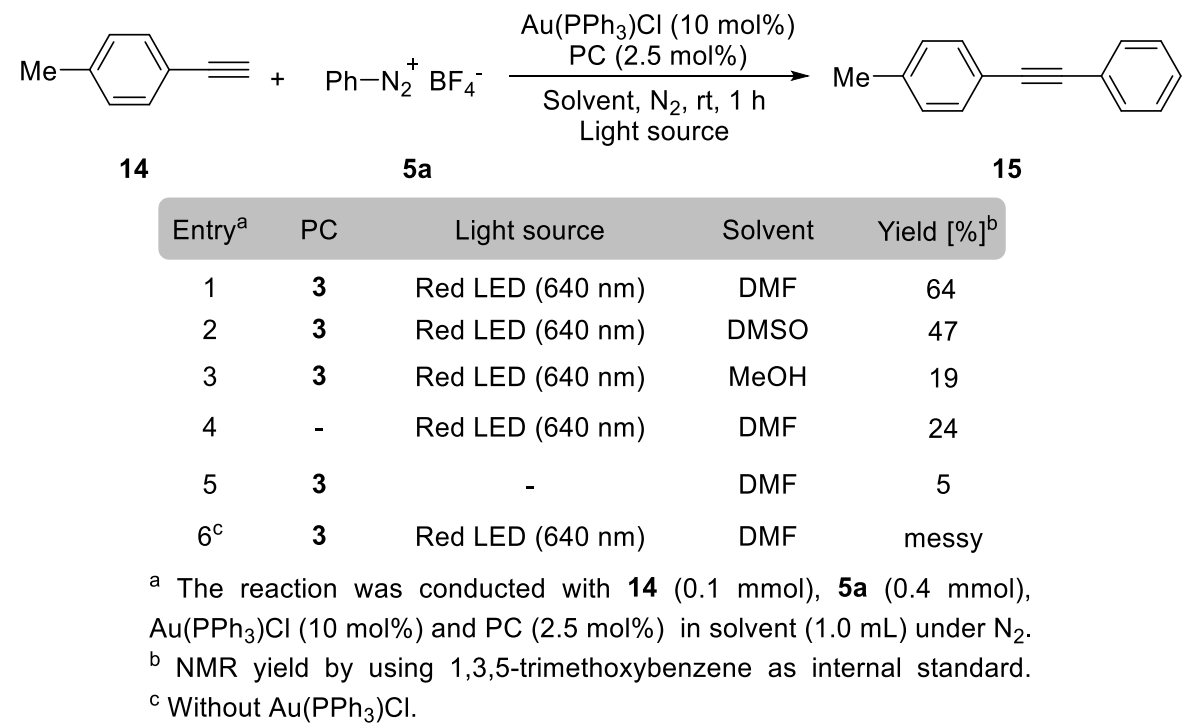

Table S7. Optimization and control experiments of red-light-mediated dual Au/nPr-DMQA+catalyzed $\mathrm{C}(\mathrm{sp})-\mathrm{H}$ arylation 


\section{NMR results for control reactions for Scheme 2}

Several control experiments have also been performed for the red-light-induced aerobic benzylic $\mathrm{C}\left(\mathrm{sp}_{3}\right)-\mathrm{H}$ oxygenation, intermolecular ATRA and dual Au/nPr-DMQA+-catalyzed C(sp)-H arylation (Scheme S2). For aerobic benzylic $\mathrm{C}\left(\mathrm{sp}_{3}\right)-\mathrm{H}$ oxygenation, no conversion was observed in the absence of PC 3 or red light, while the reaction was messy in the absence of DBU (Scheme S2a). For intermolecular ATRA, no reaction occurred in the absence of PC 3 or red light, while the desired adduct 13 was obtained in 14\% yield along with 75\% of SM-11 in the absence of LiBr (Scheme $\mathrm{S} 2 \mathrm{~b})$. For dual $\mathrm{Au} /{ }_{n} \mathrm{Pr}-\mathrm{DMQA}+-$ catalyzed $\mathrm{C}(\mathrm{sp})-\mathrm{H}$ arylation, in the absence of $\mathrm{PC} \mathbf{3}$ or red light, the desired product 15 was formed in $24 \%$ and $5 \%$ yield, respectively, while the reaction was messy in the absence of $\mathrm{Au}\left(\mathrm{PPh}_{3}\right) \mathrm{Cl}$ (Scheme $\left.\mathrm{S} 2 \mathrm{c}\right)$.

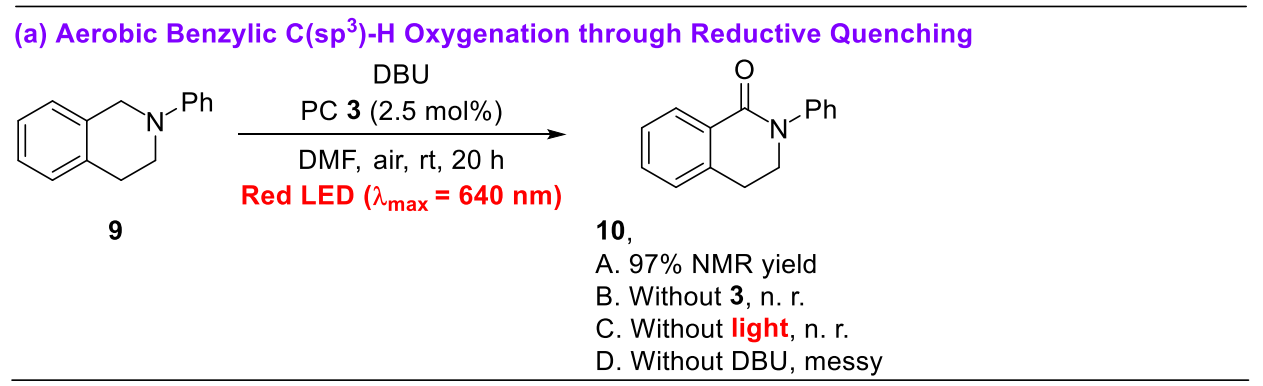

(b) Intermolecular Atom Transfer Radical Addition (ATRA) through Oxidative Quenching

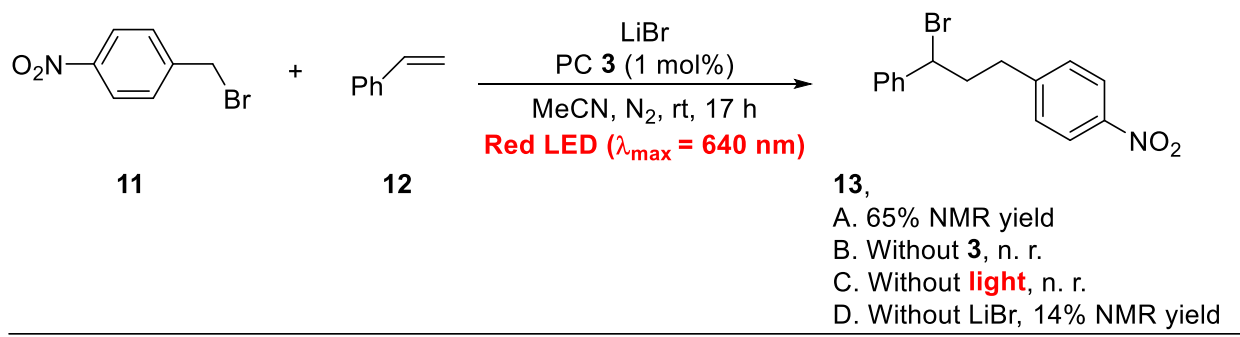

(c) Dual Au/ ${ }^{\mathrm{P}} \mathrm{Pr}-\mathrm{DMQA}{ }^{+}$-Catalyzed C(sp)-H Arylation through Oxidative Quenching
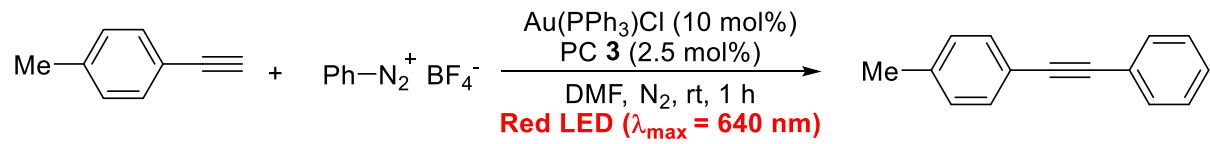

14

$5 a$

15 ,

A. $64 \%$ NMR yield

B. Without $3,24 \%$ NMR yield

C. Without light, $5 \%$ NMR yield

D. Without $\mathrm{Au}\left(\mathrm{PPh}_{3}\right) \mathrm{Cl}$, messy

${ }^{a}$ Reactions were conducted on $0.1 \mathrm{mmol}$ scale. NMR yields by using 1,3,5-trimethoxybenzene as internal standard were shown.

Scheme S2. NMR results for control reactions for Scheme 2

17. NMR spectra charts for compounds $3,6,8,10,13$, and 15 


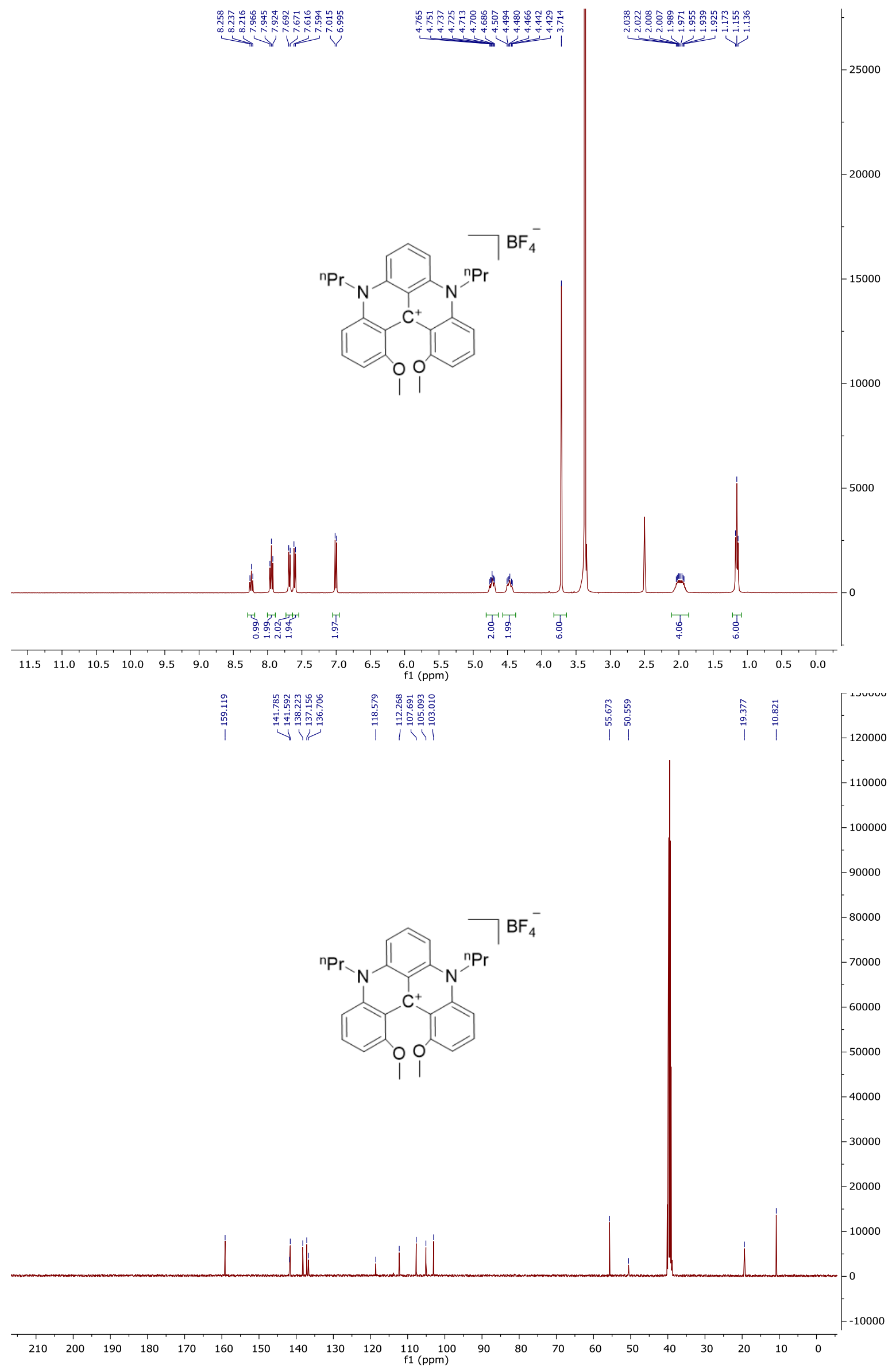




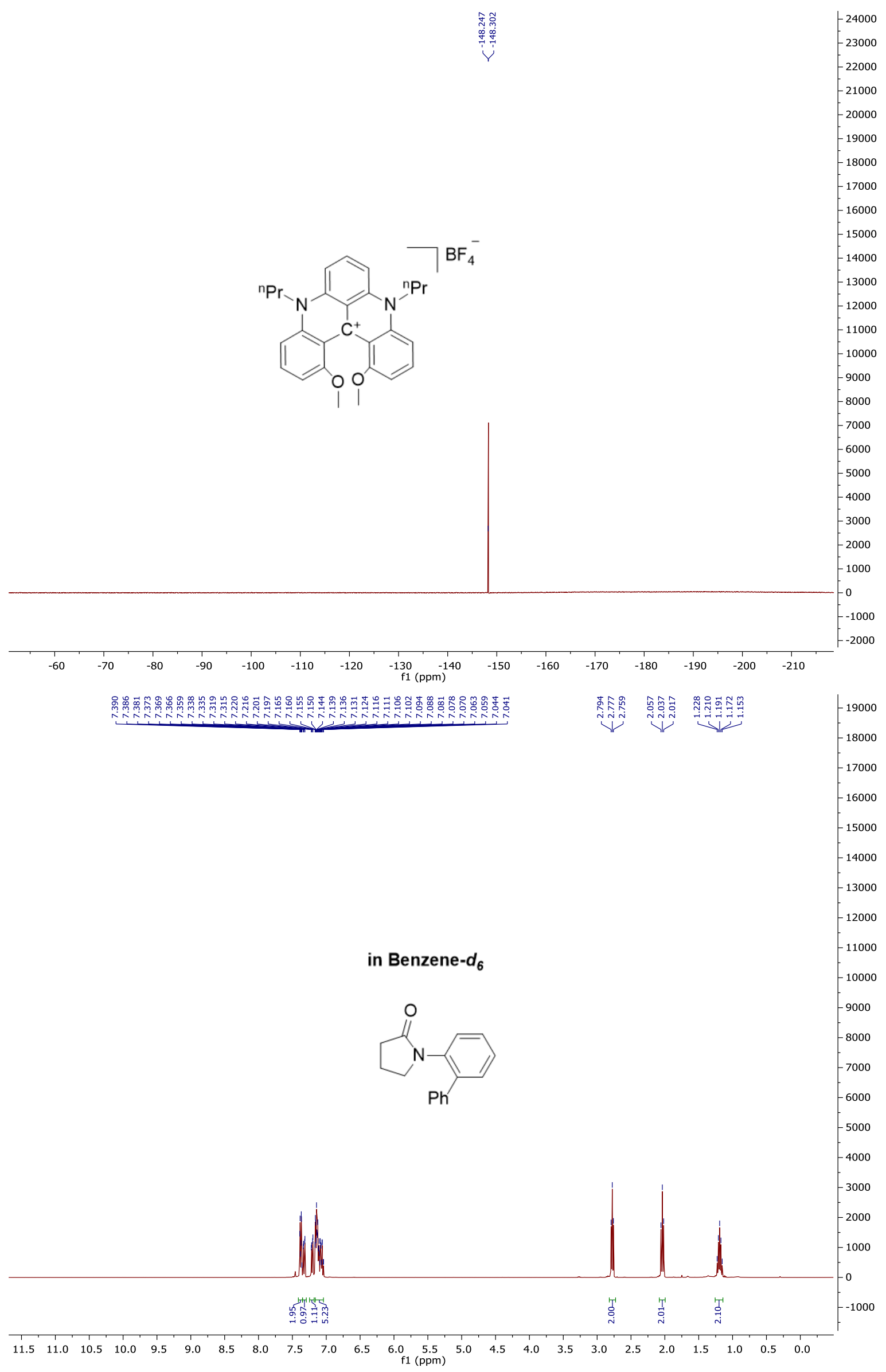




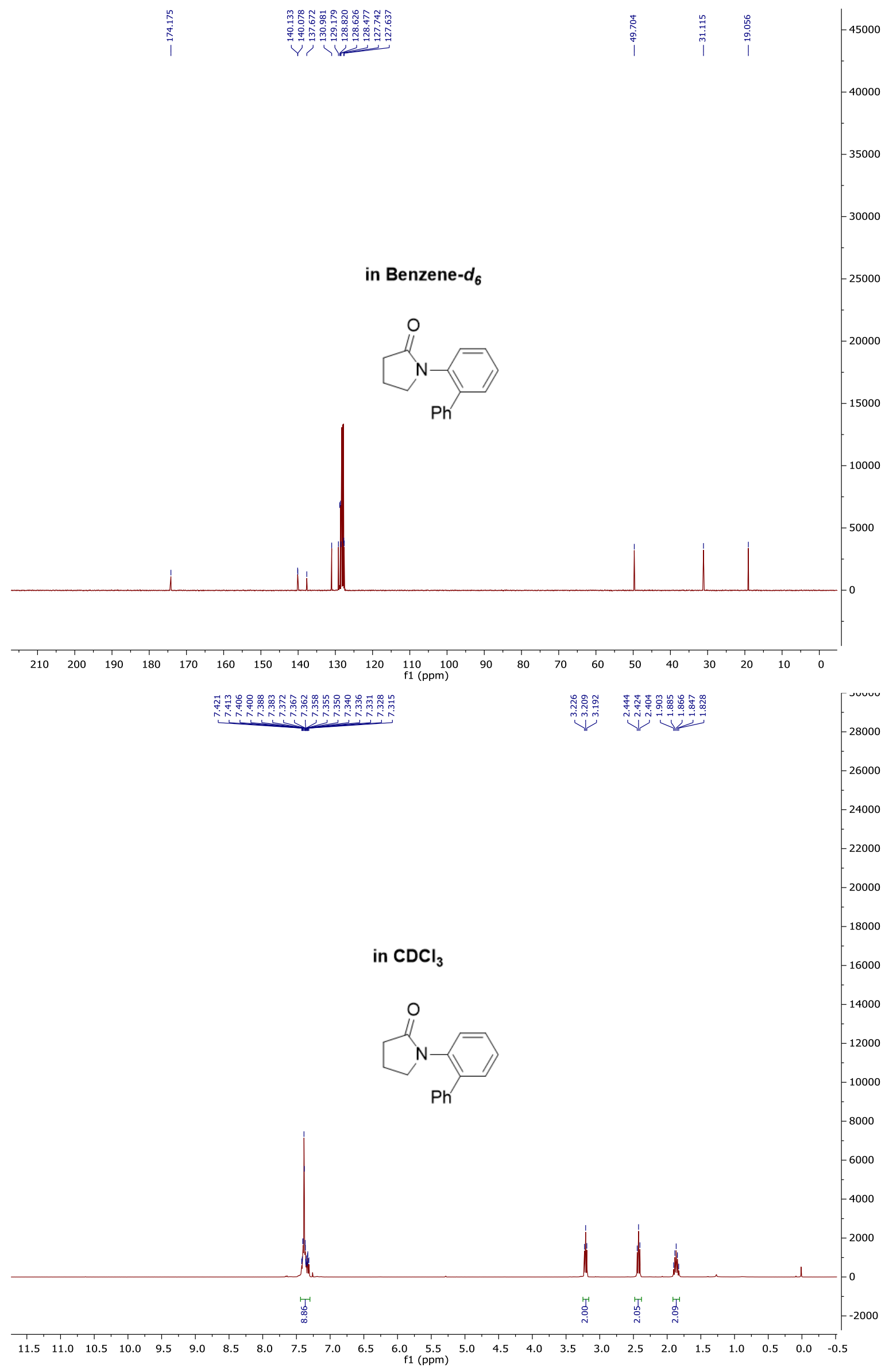




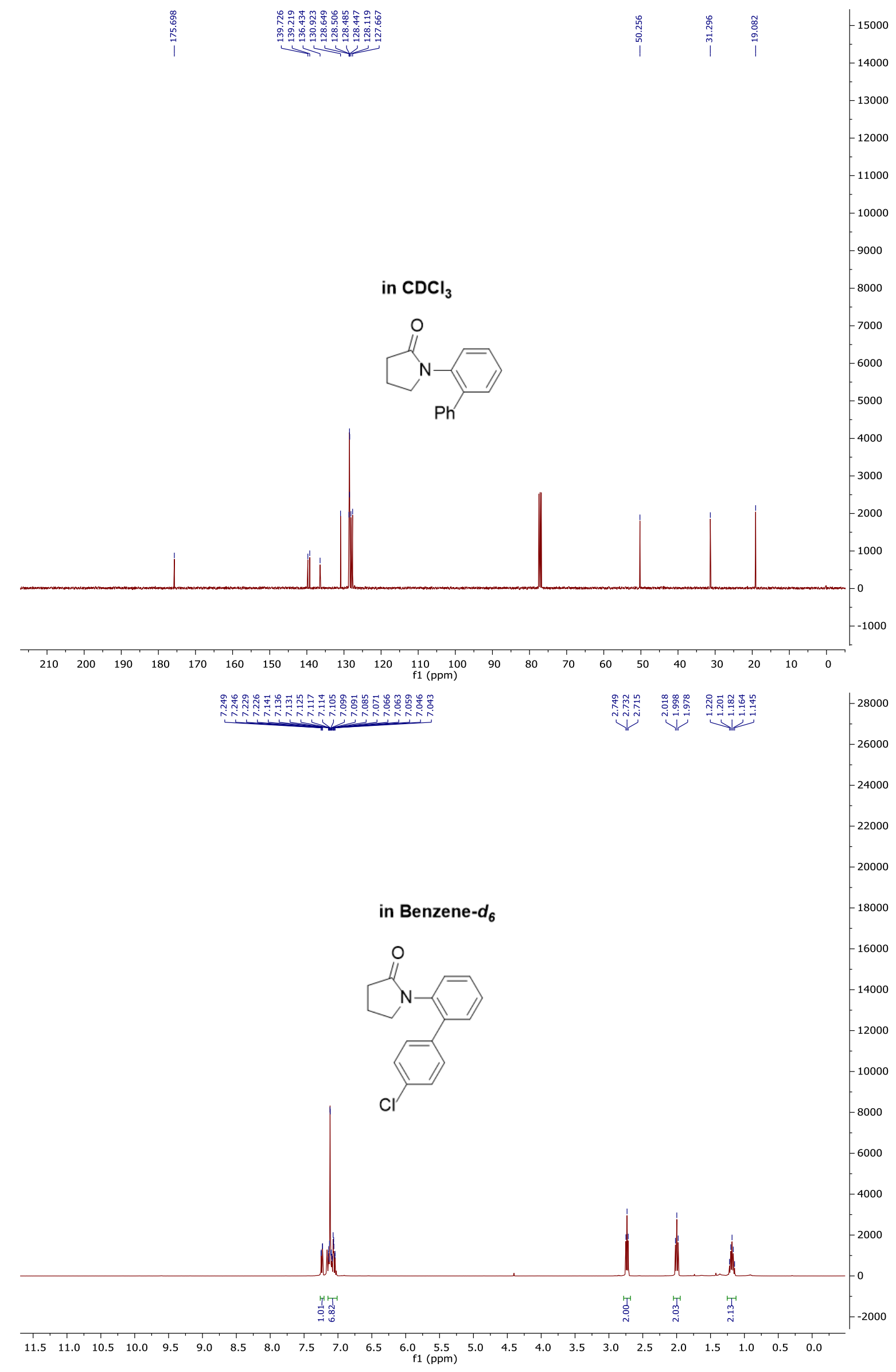




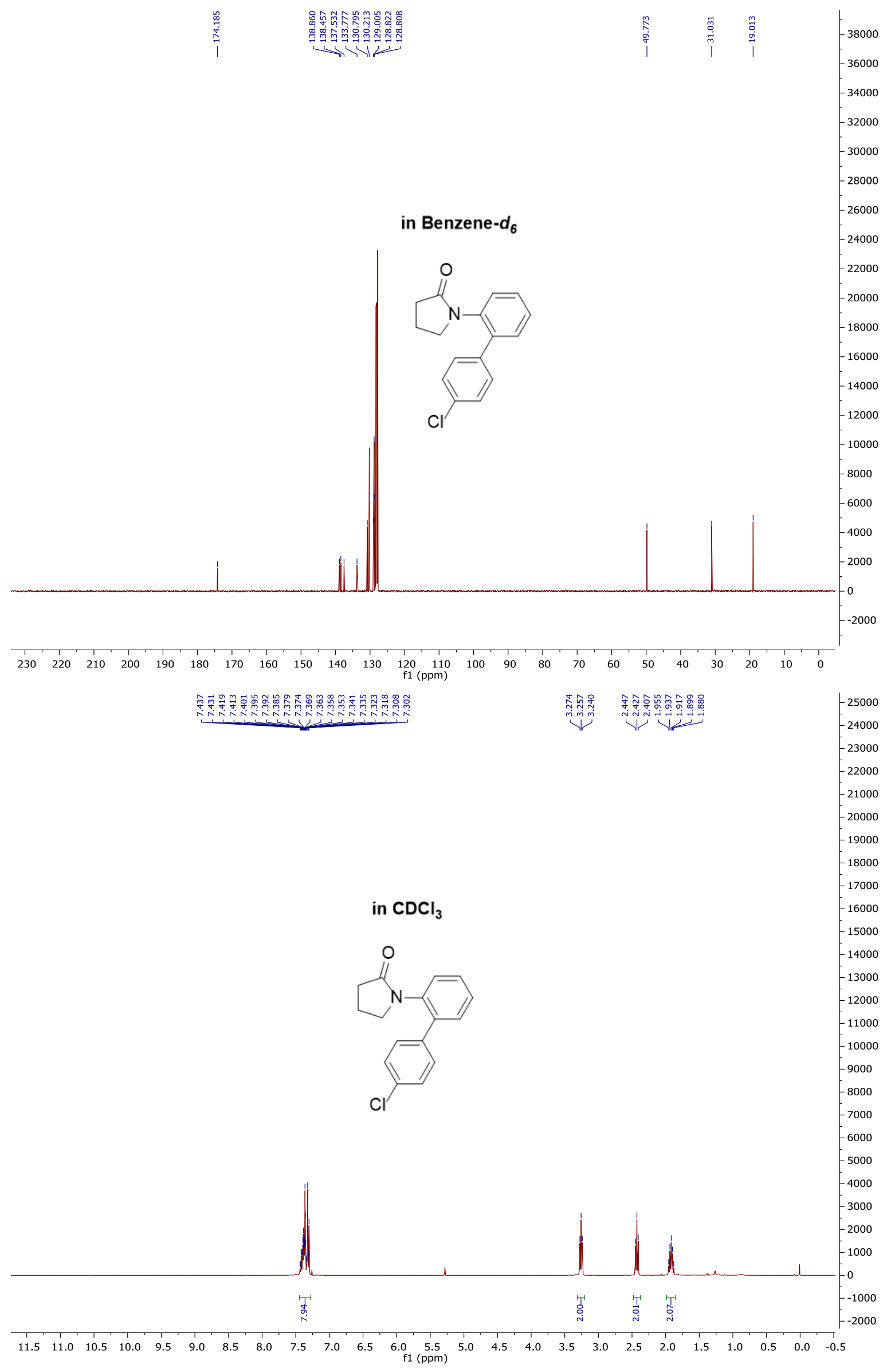




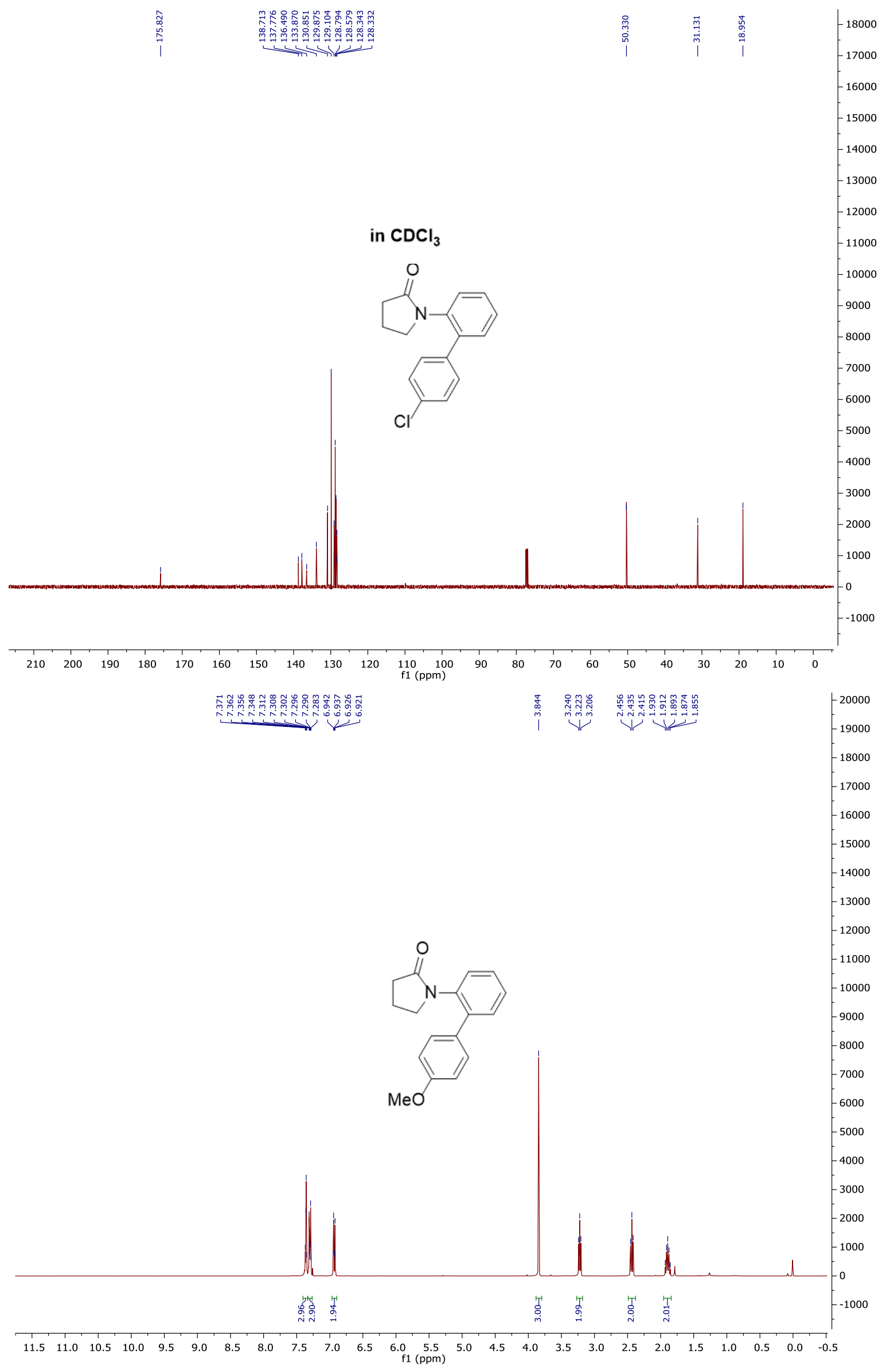




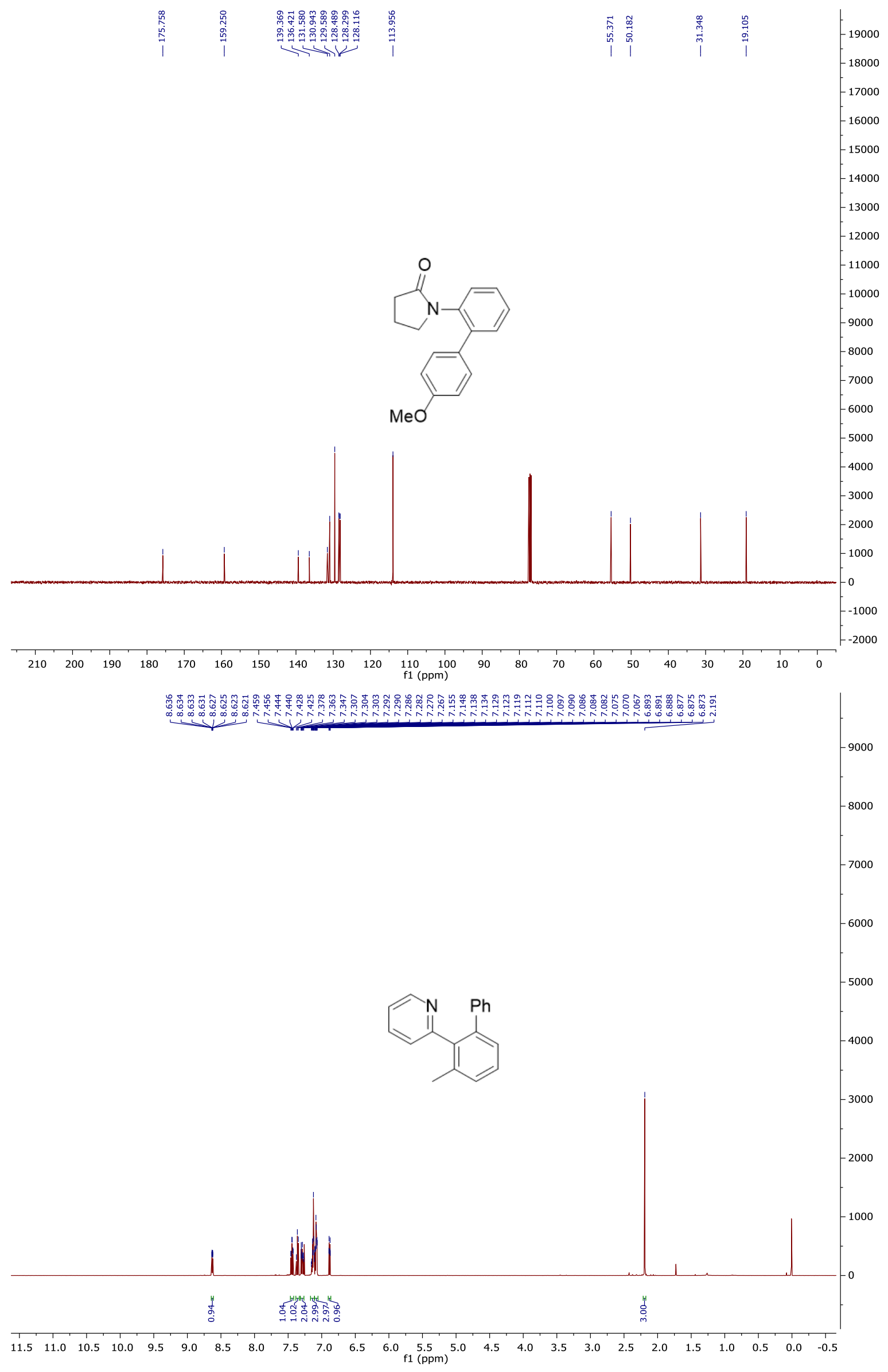




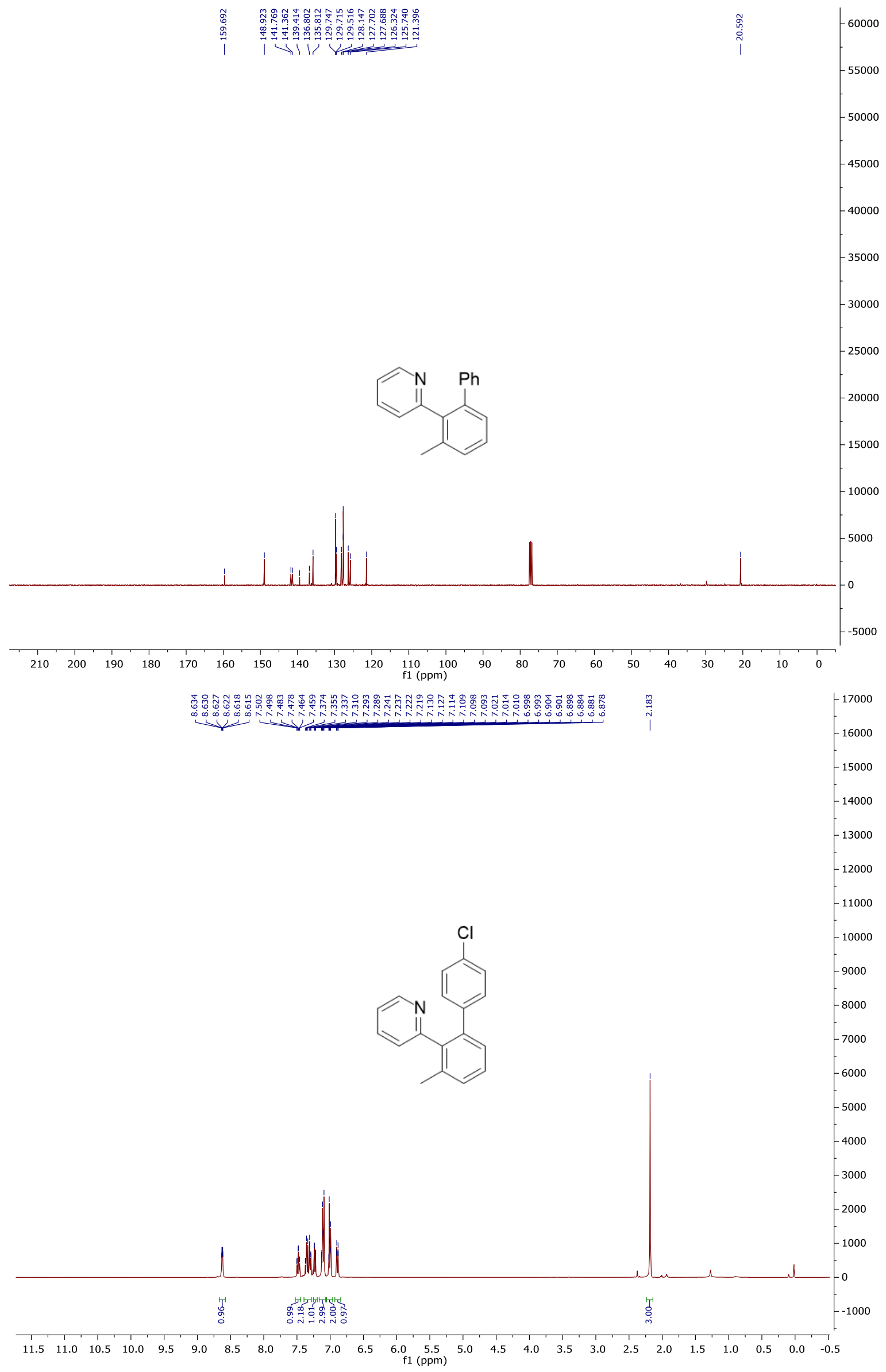




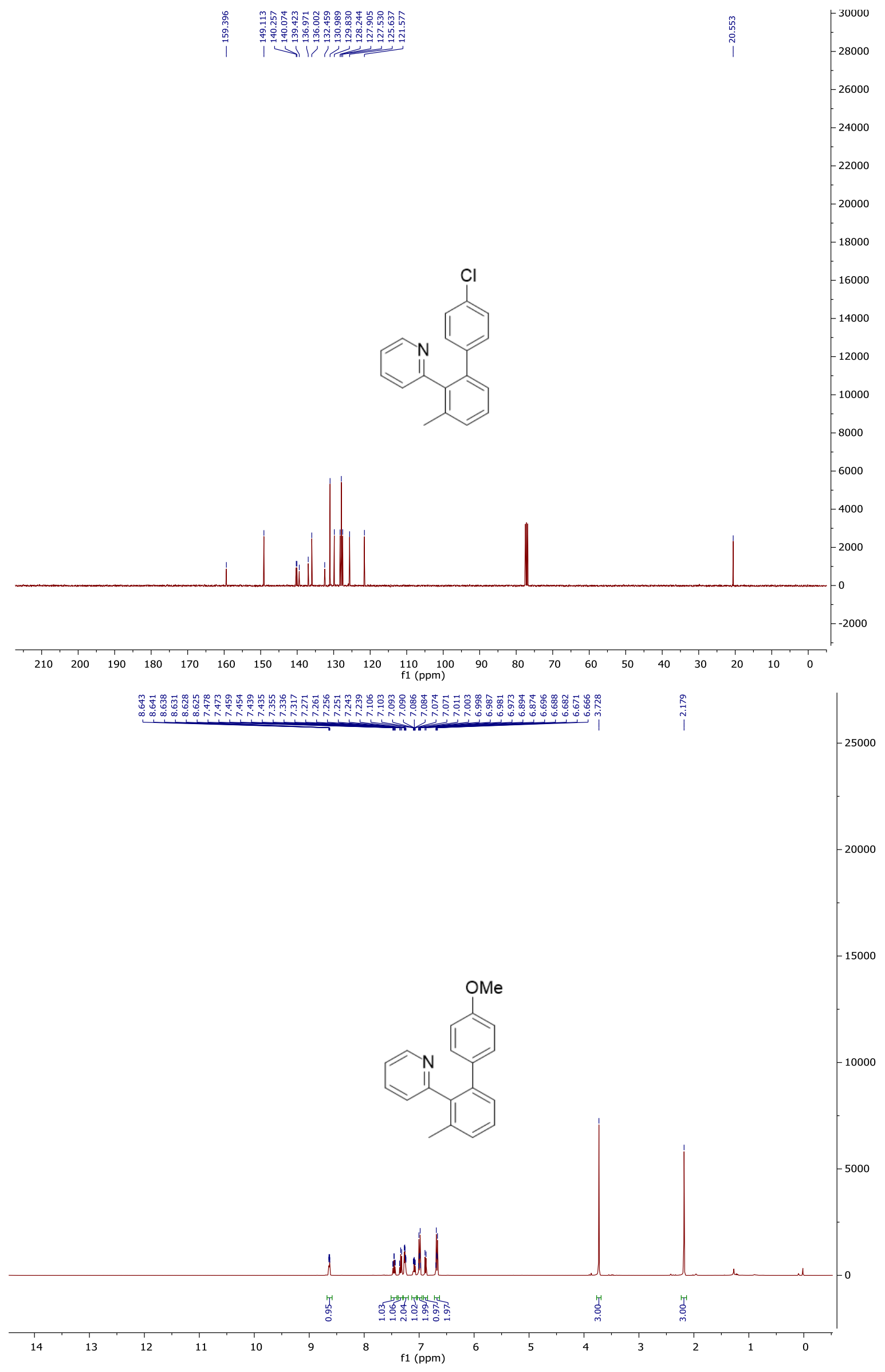




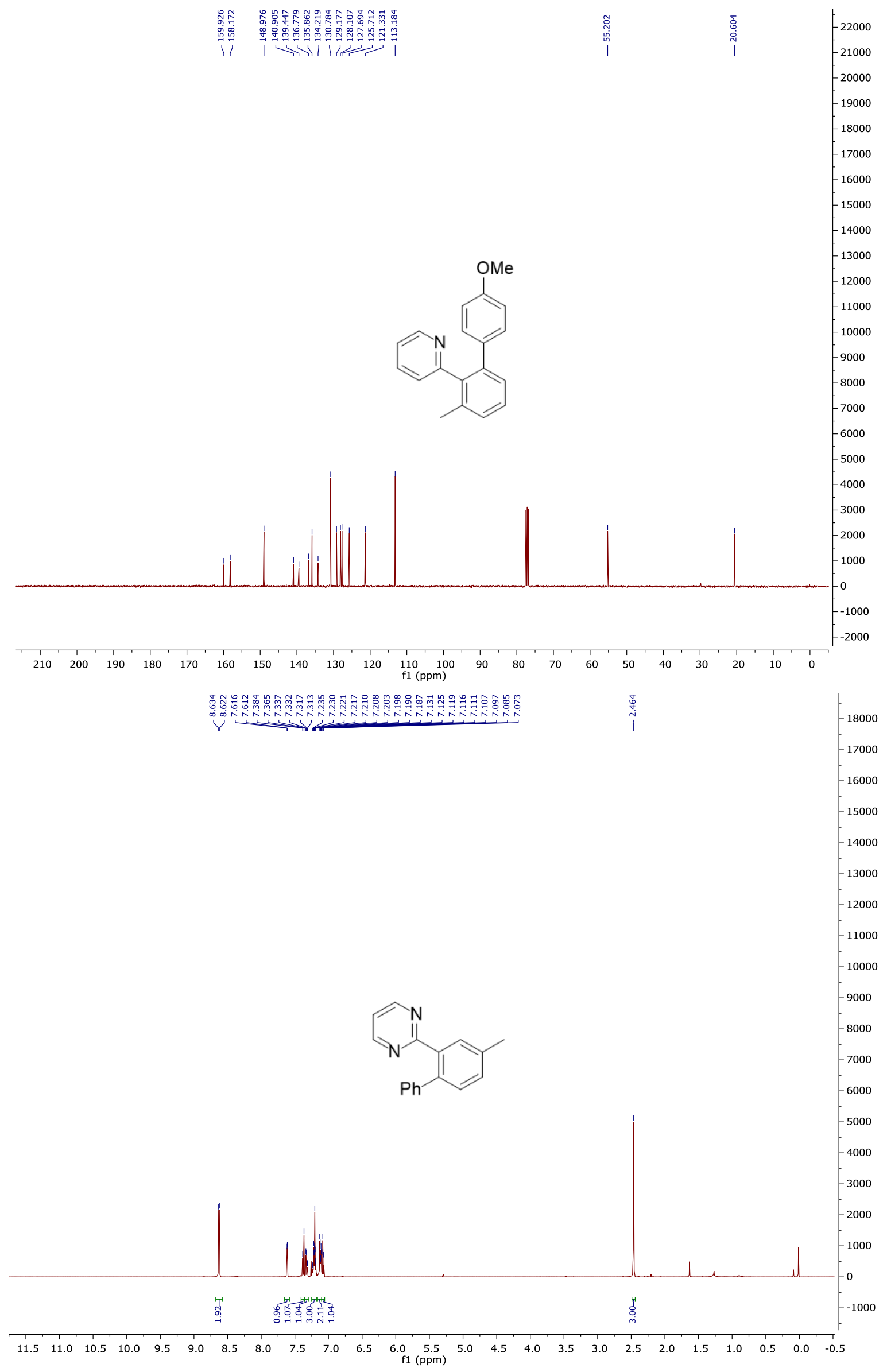




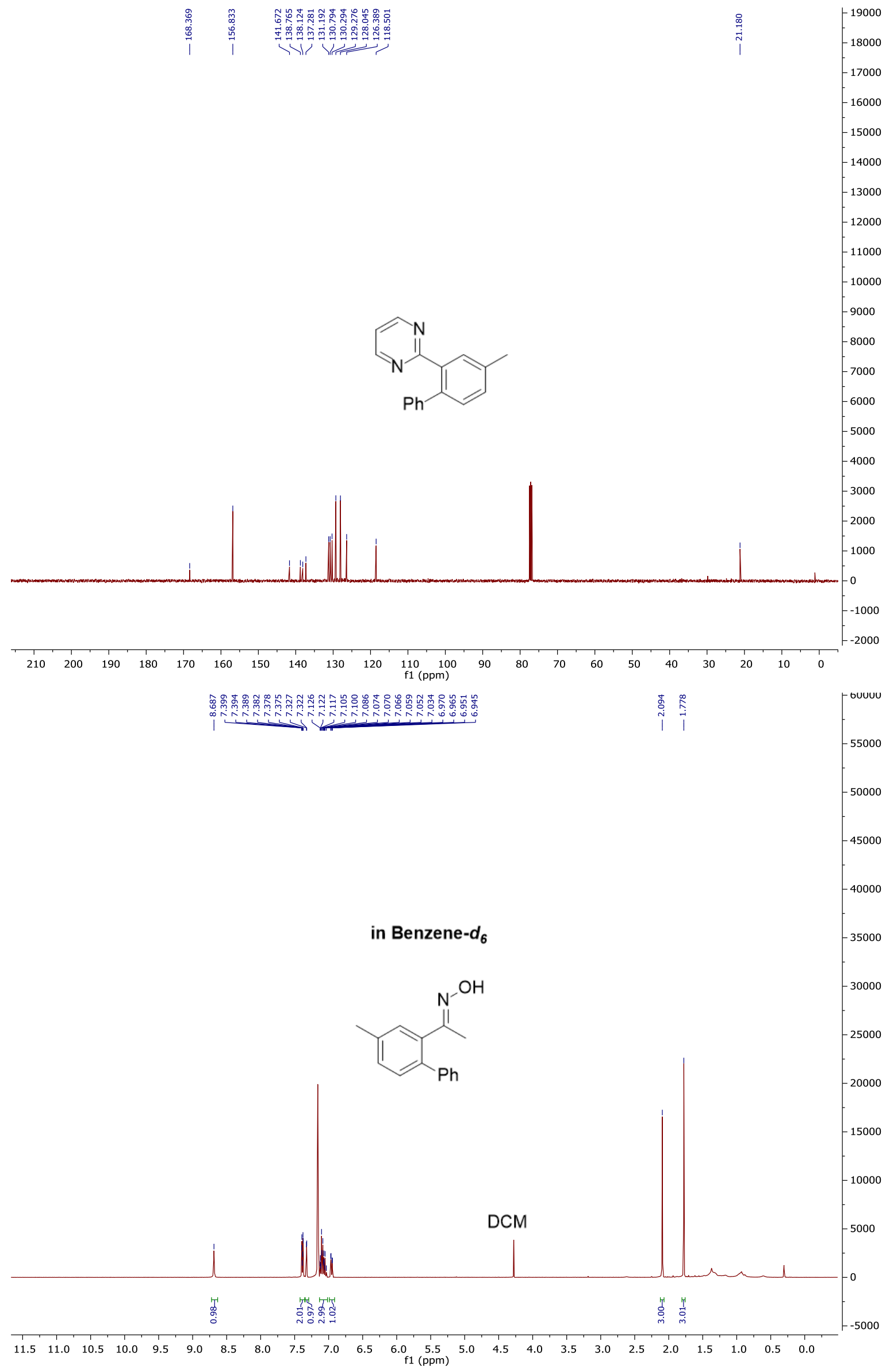




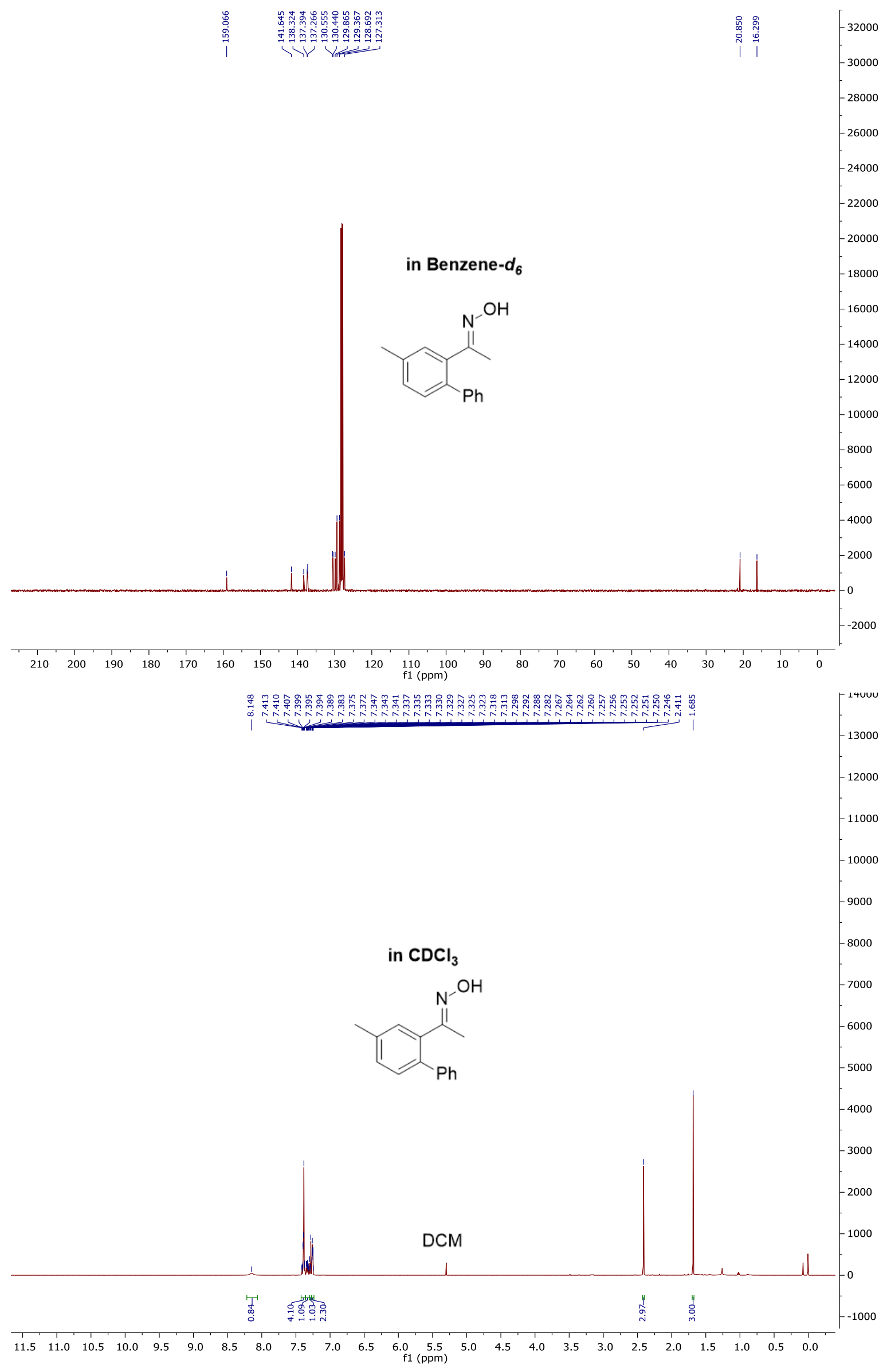




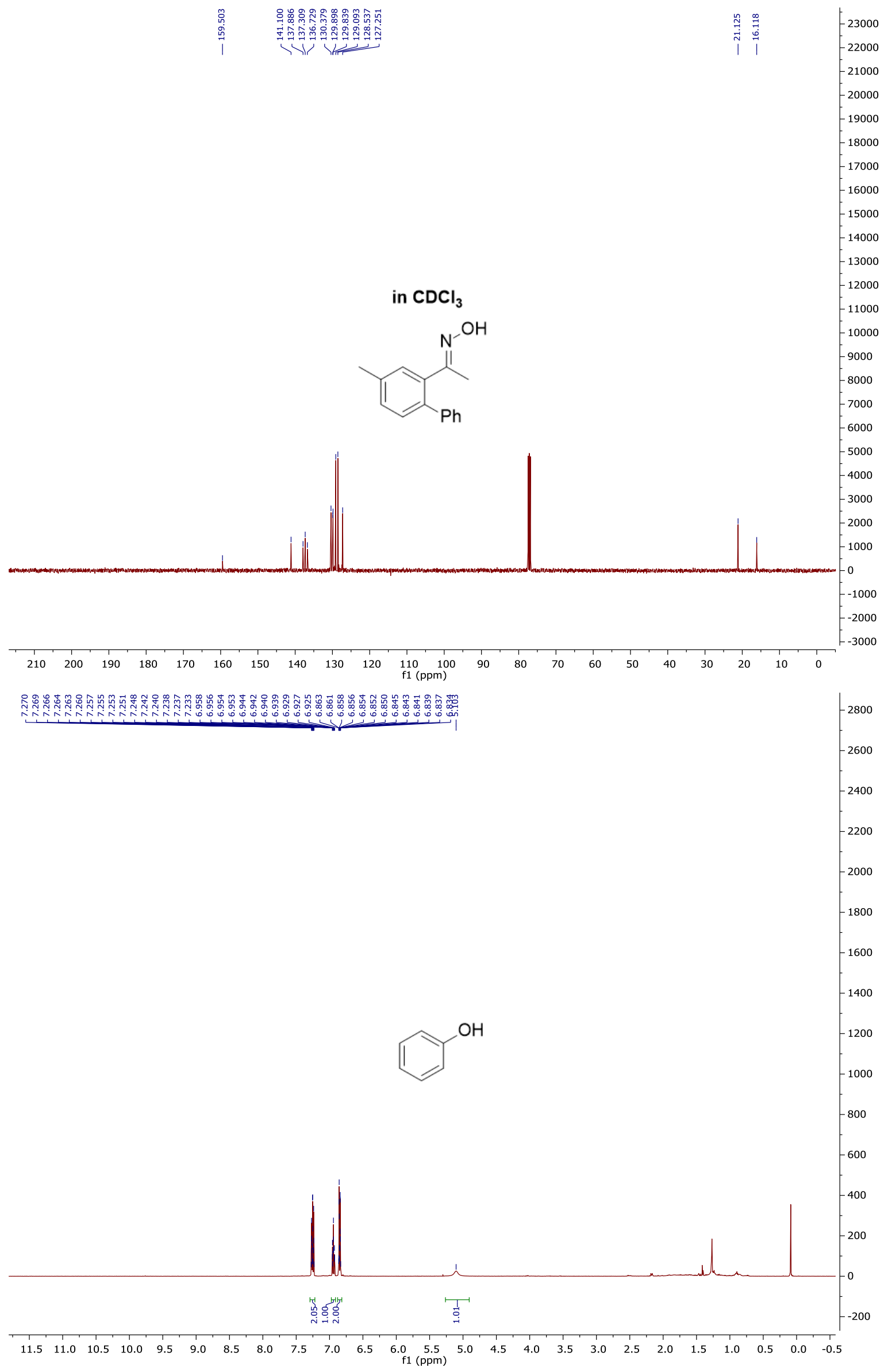




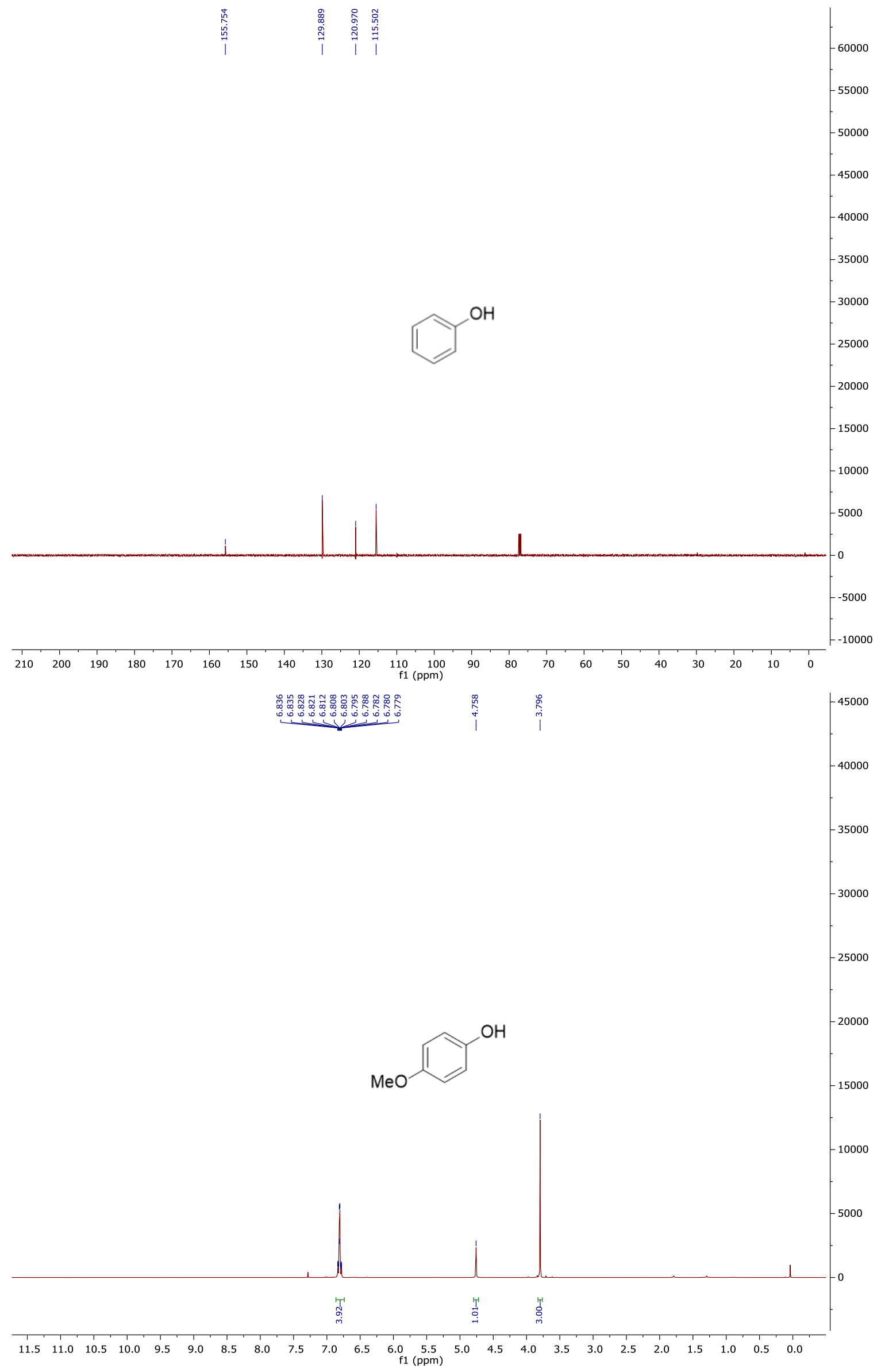




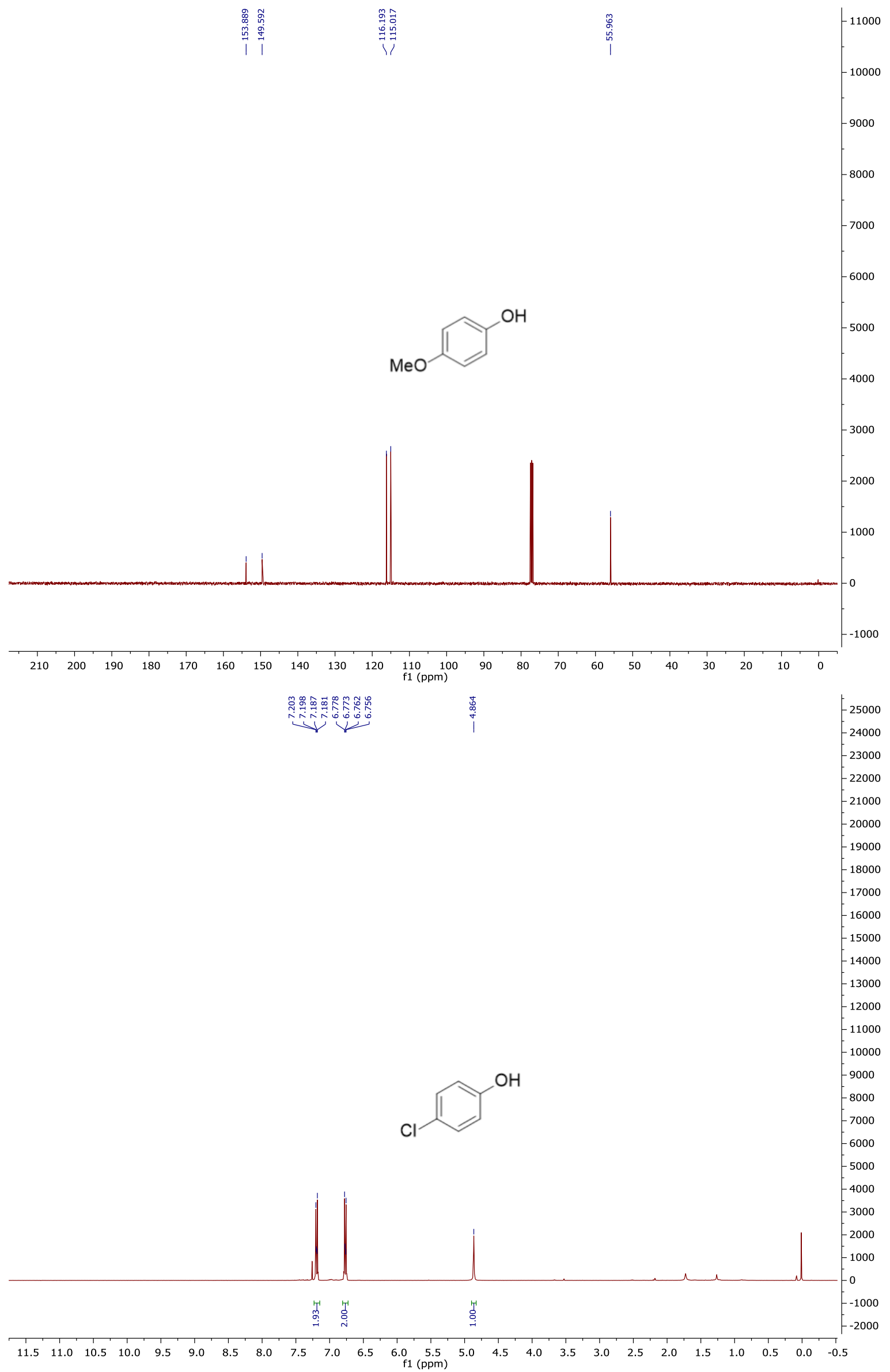




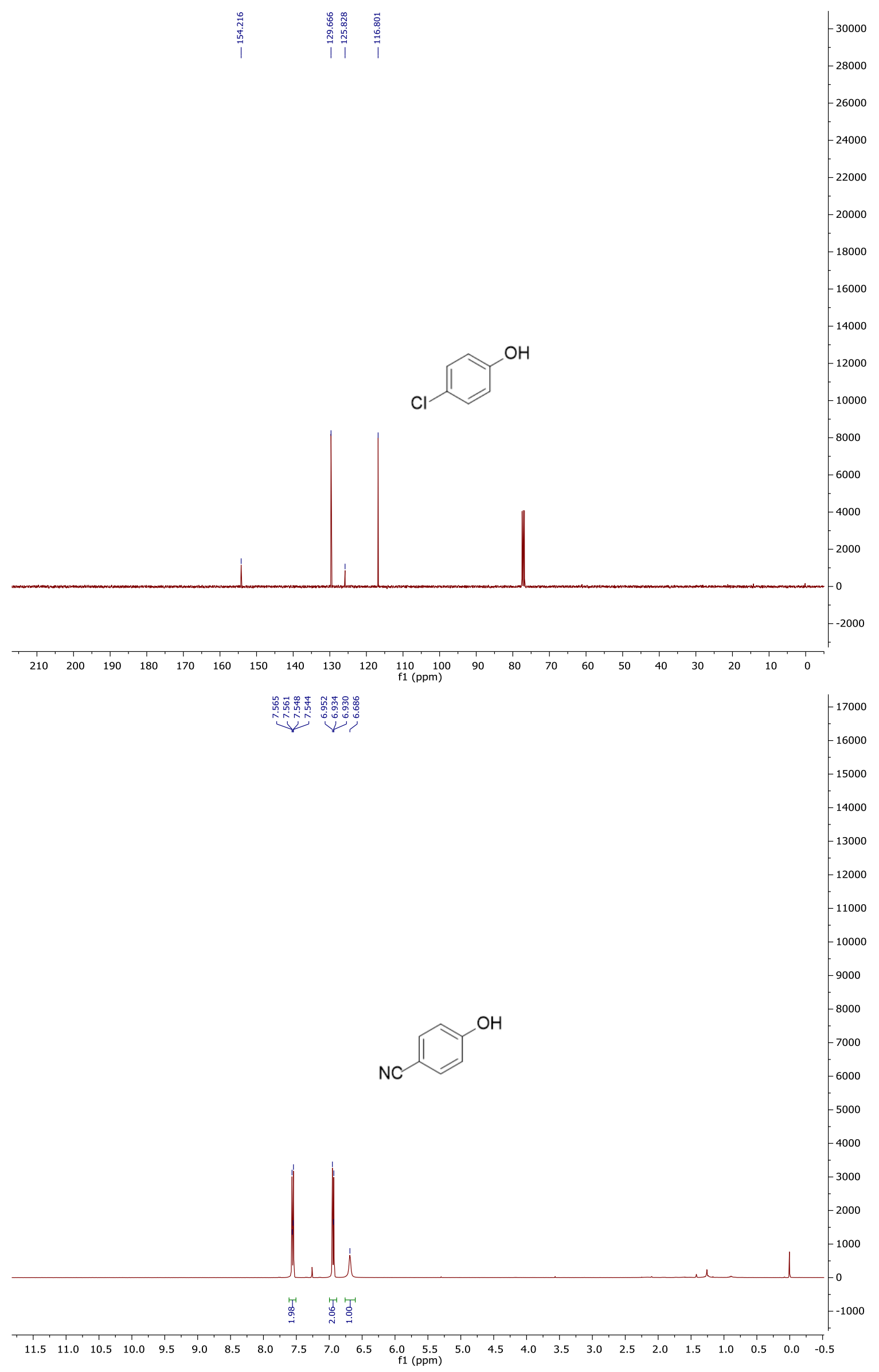




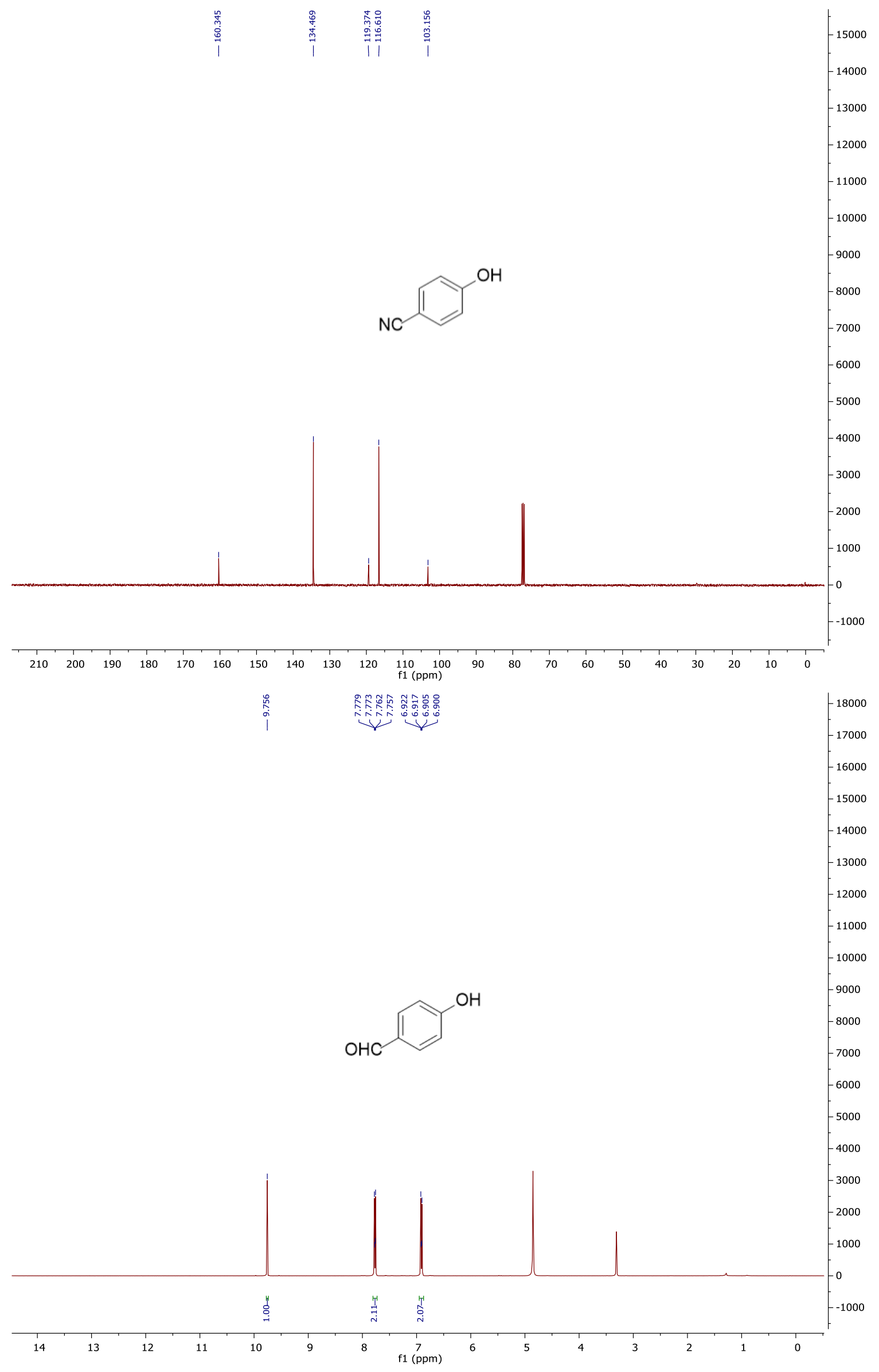




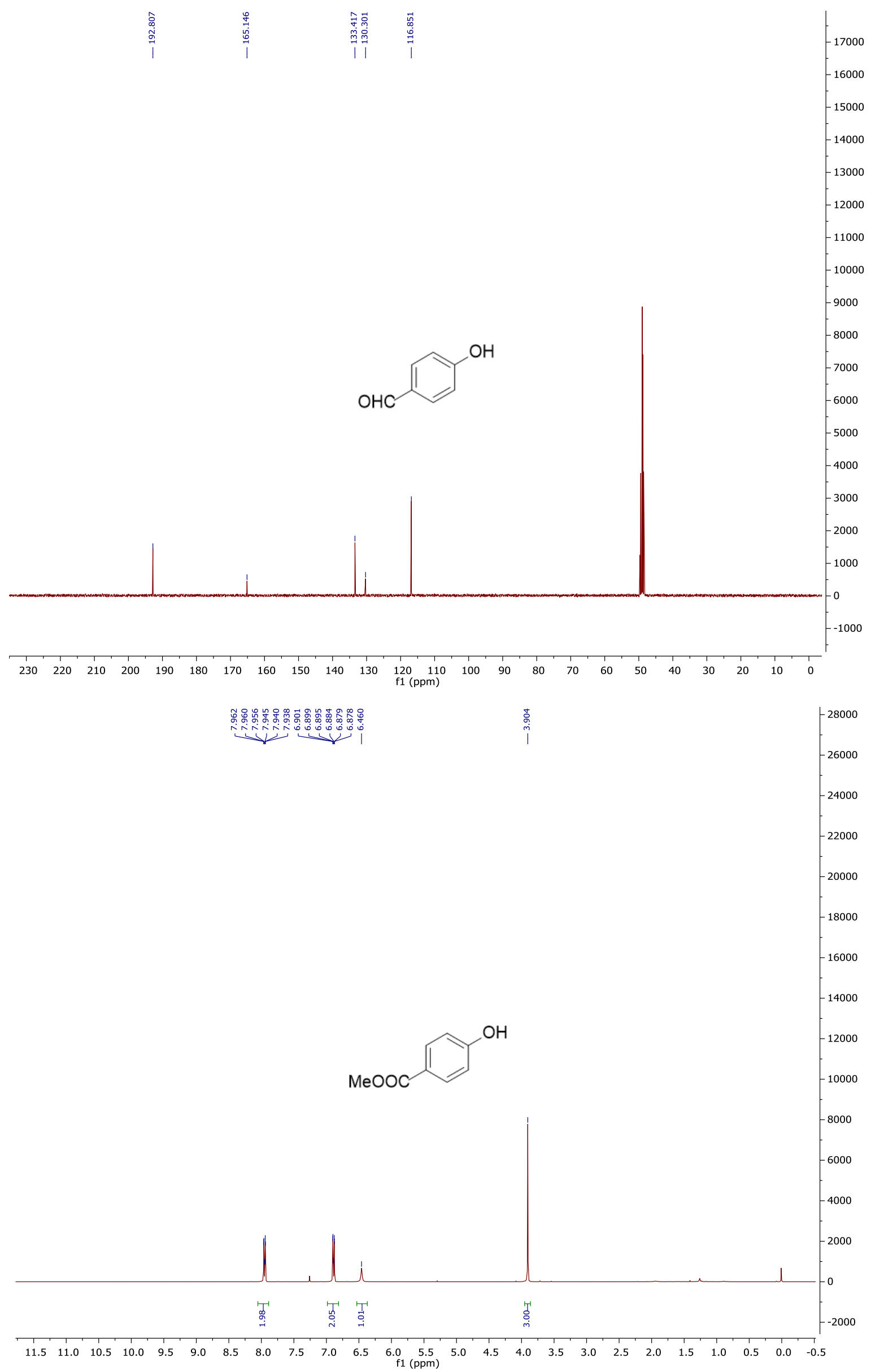




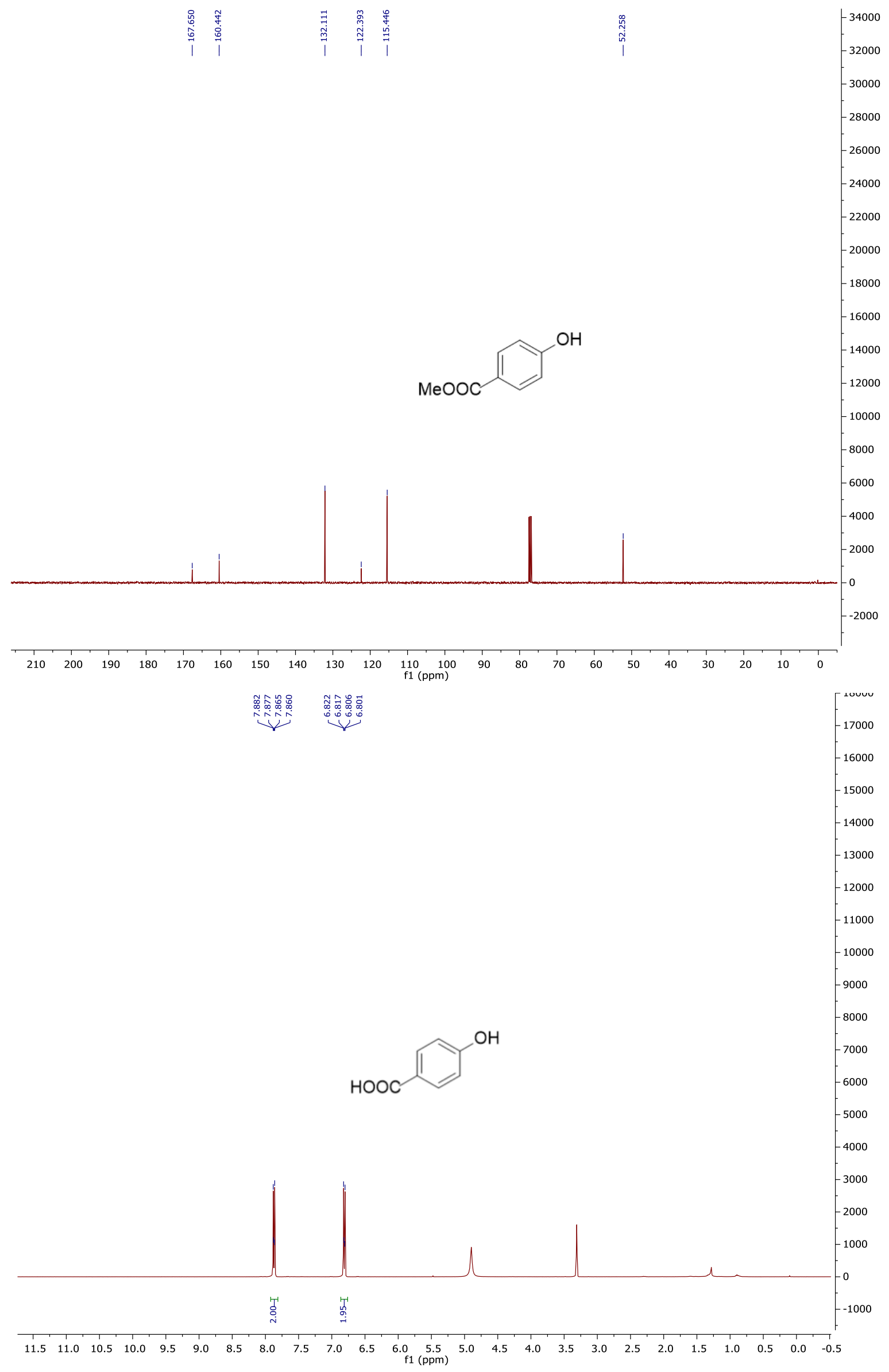




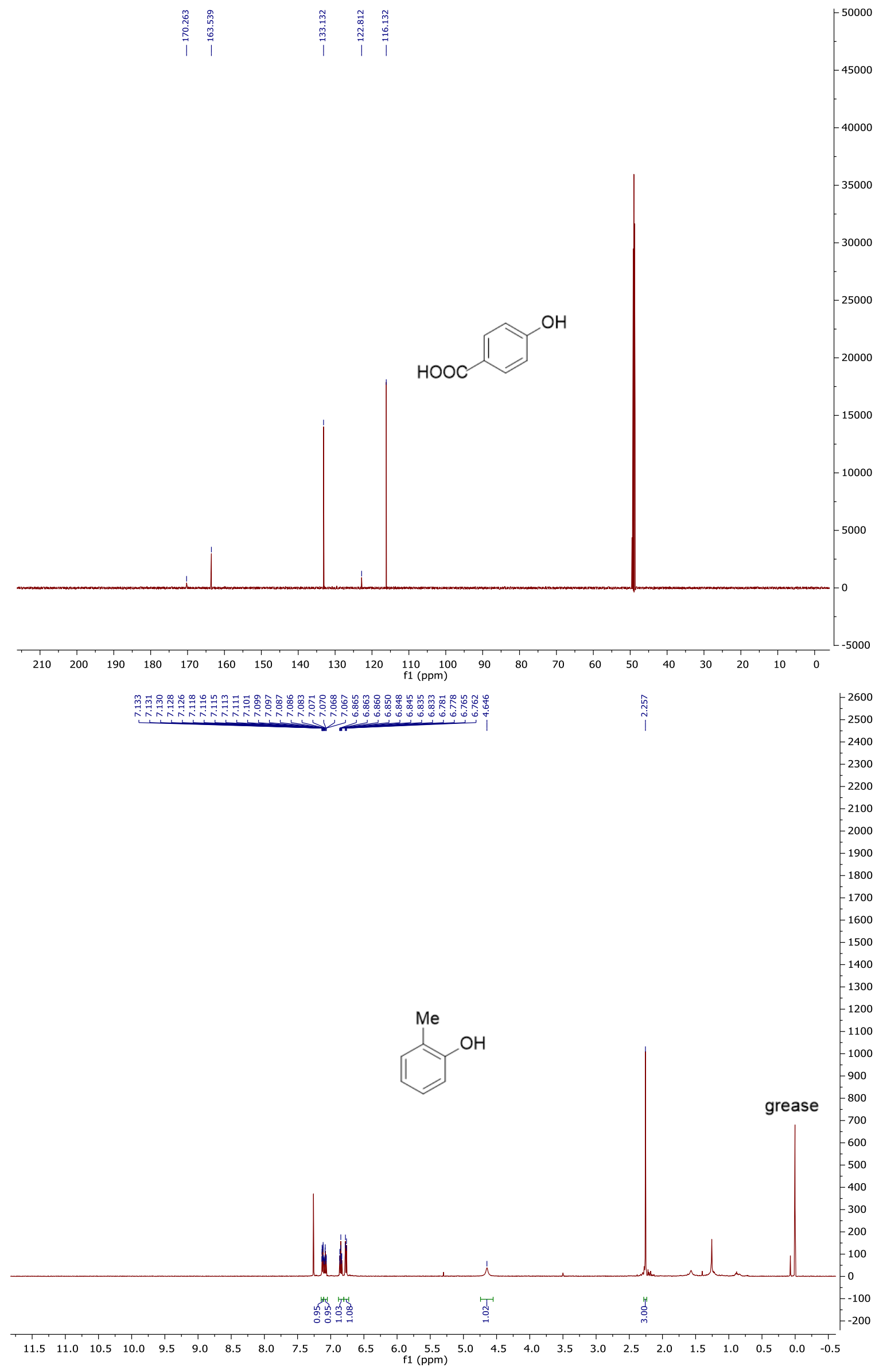




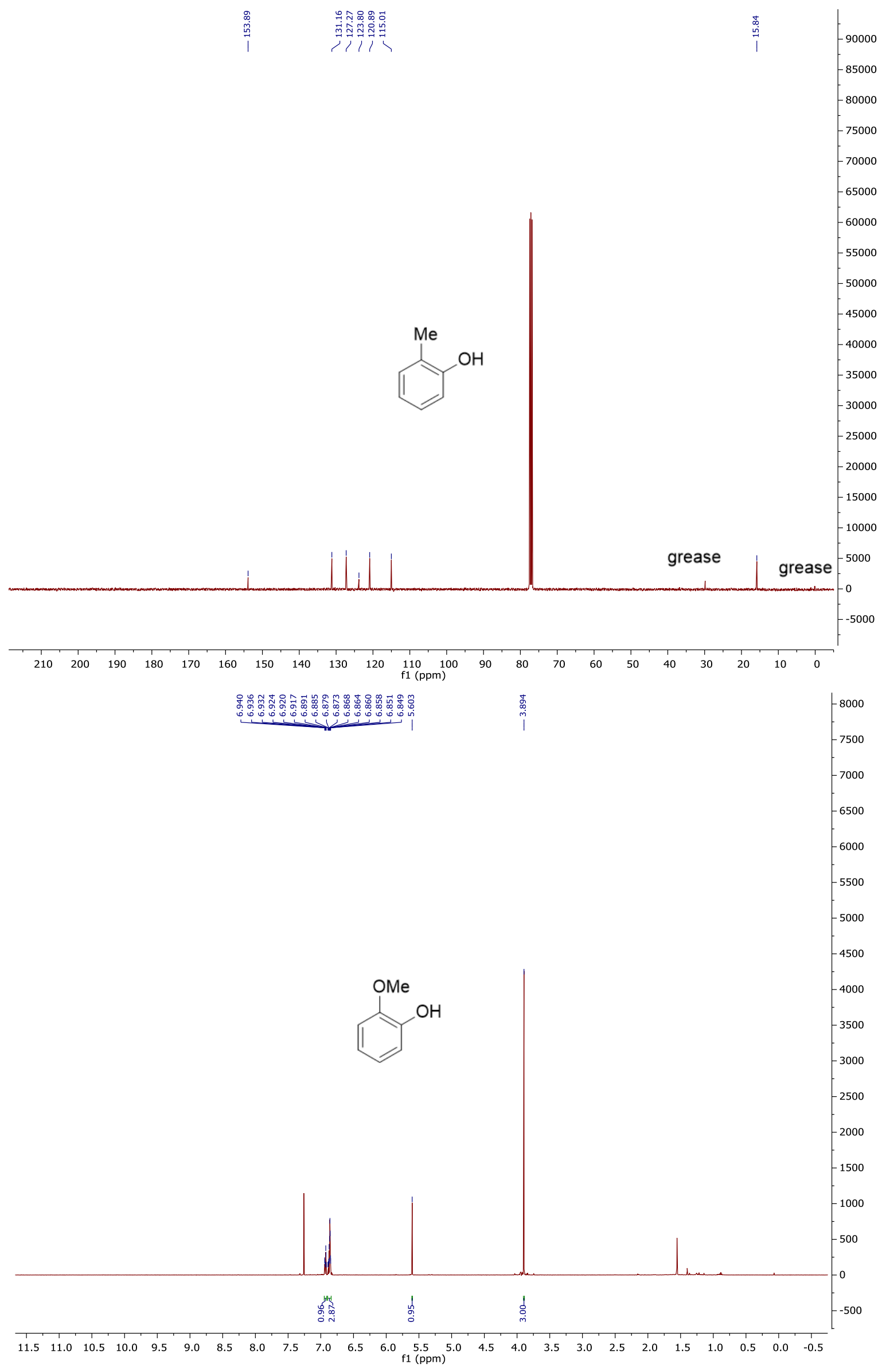




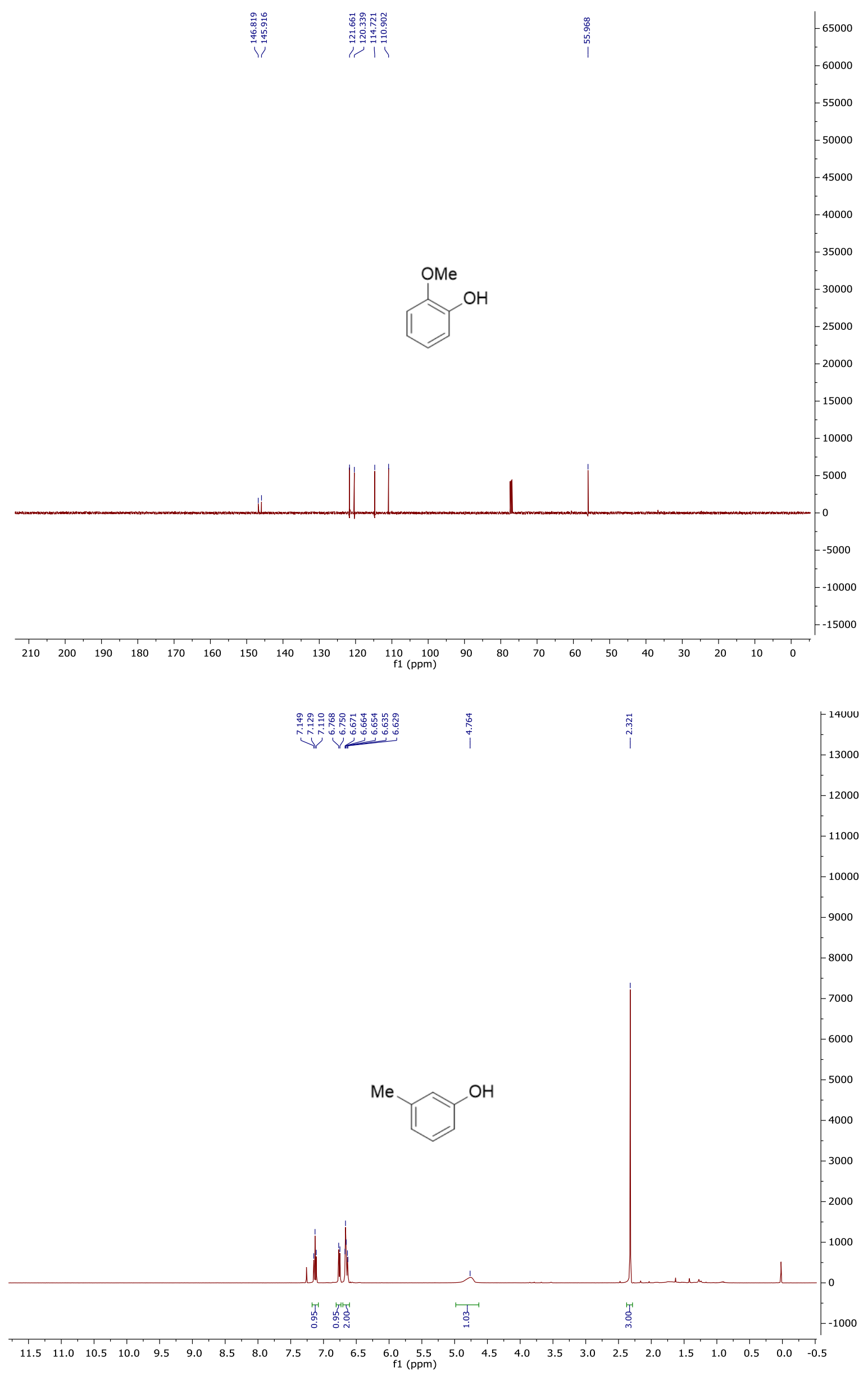




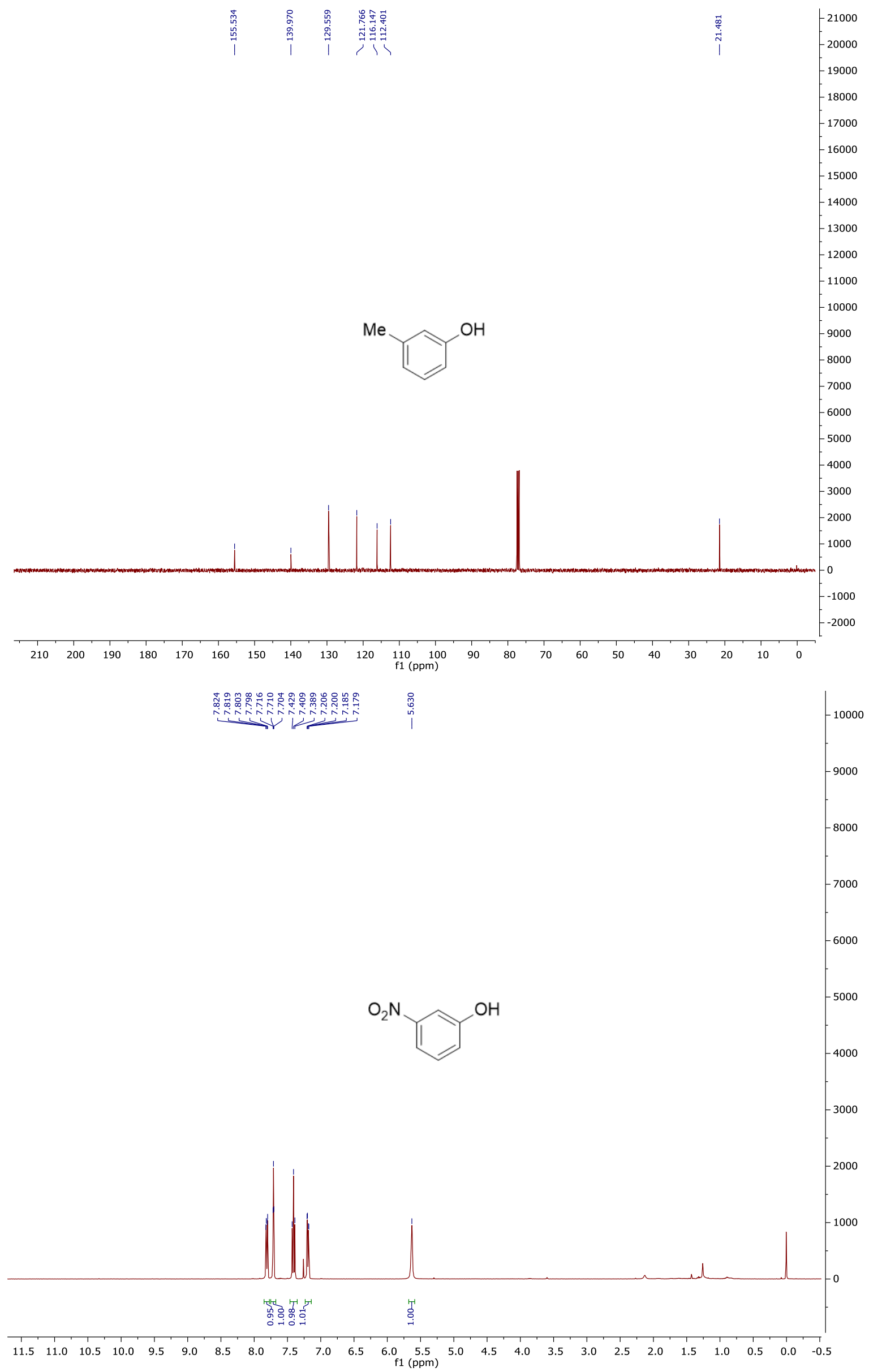




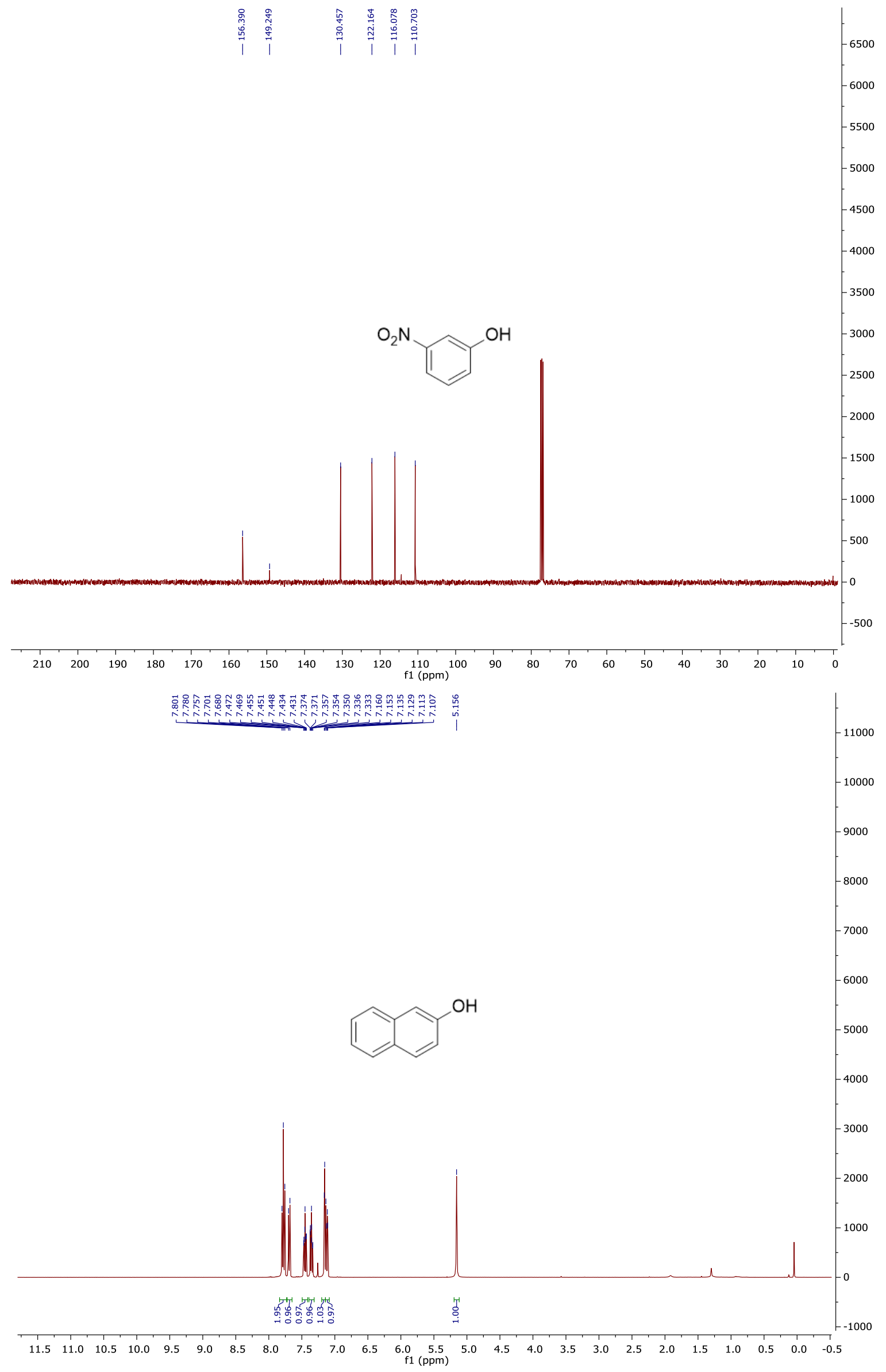




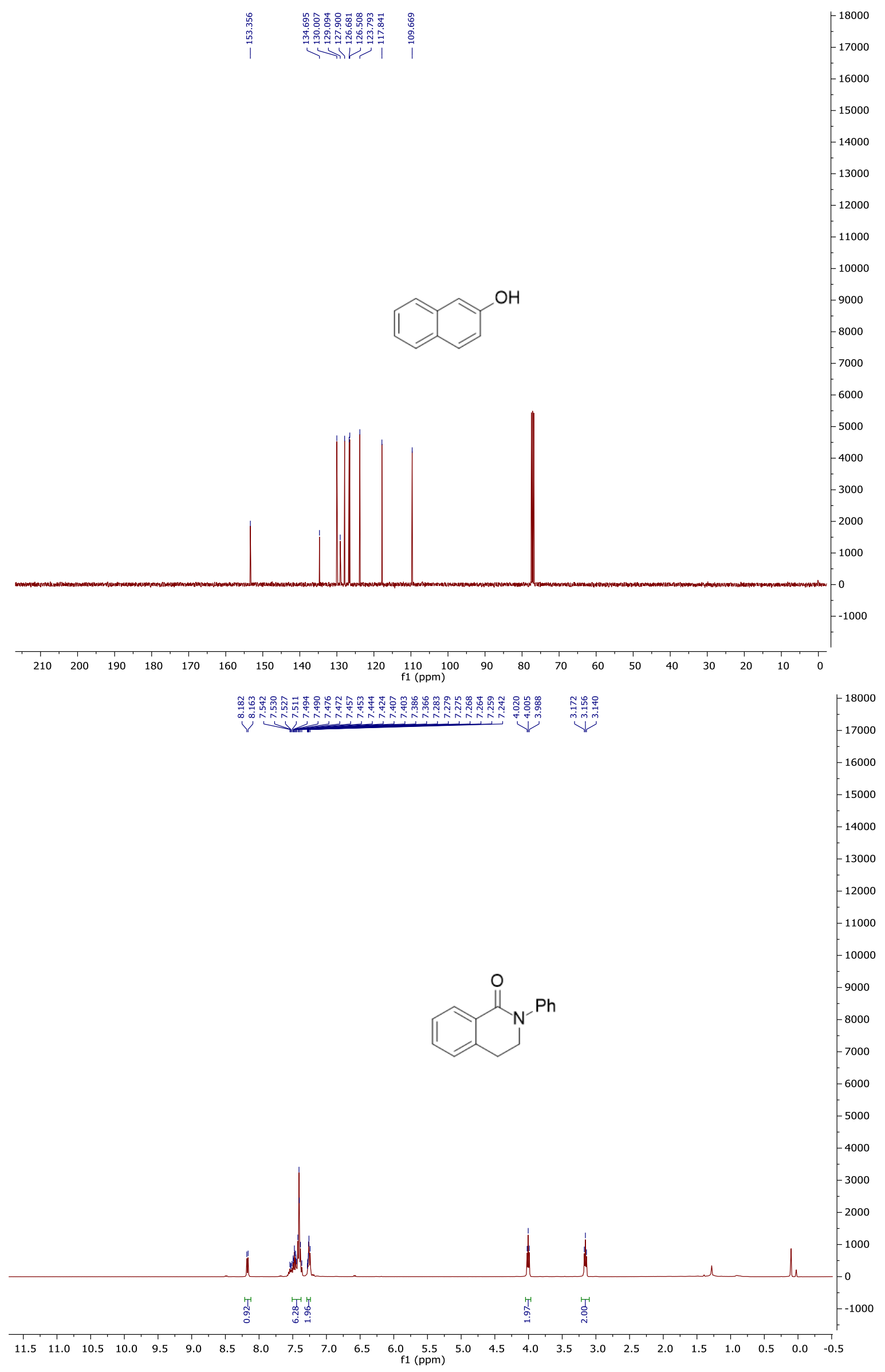




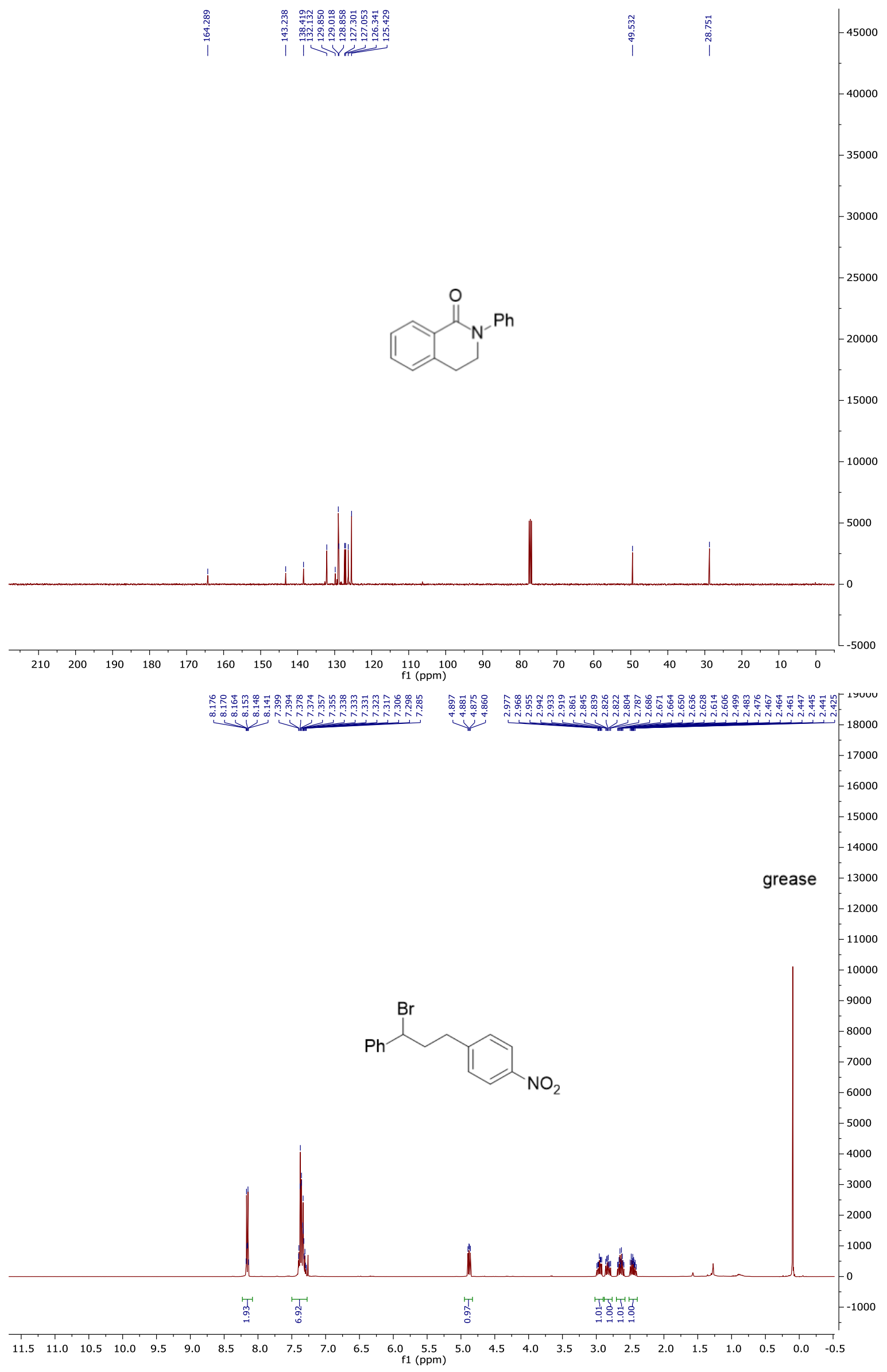




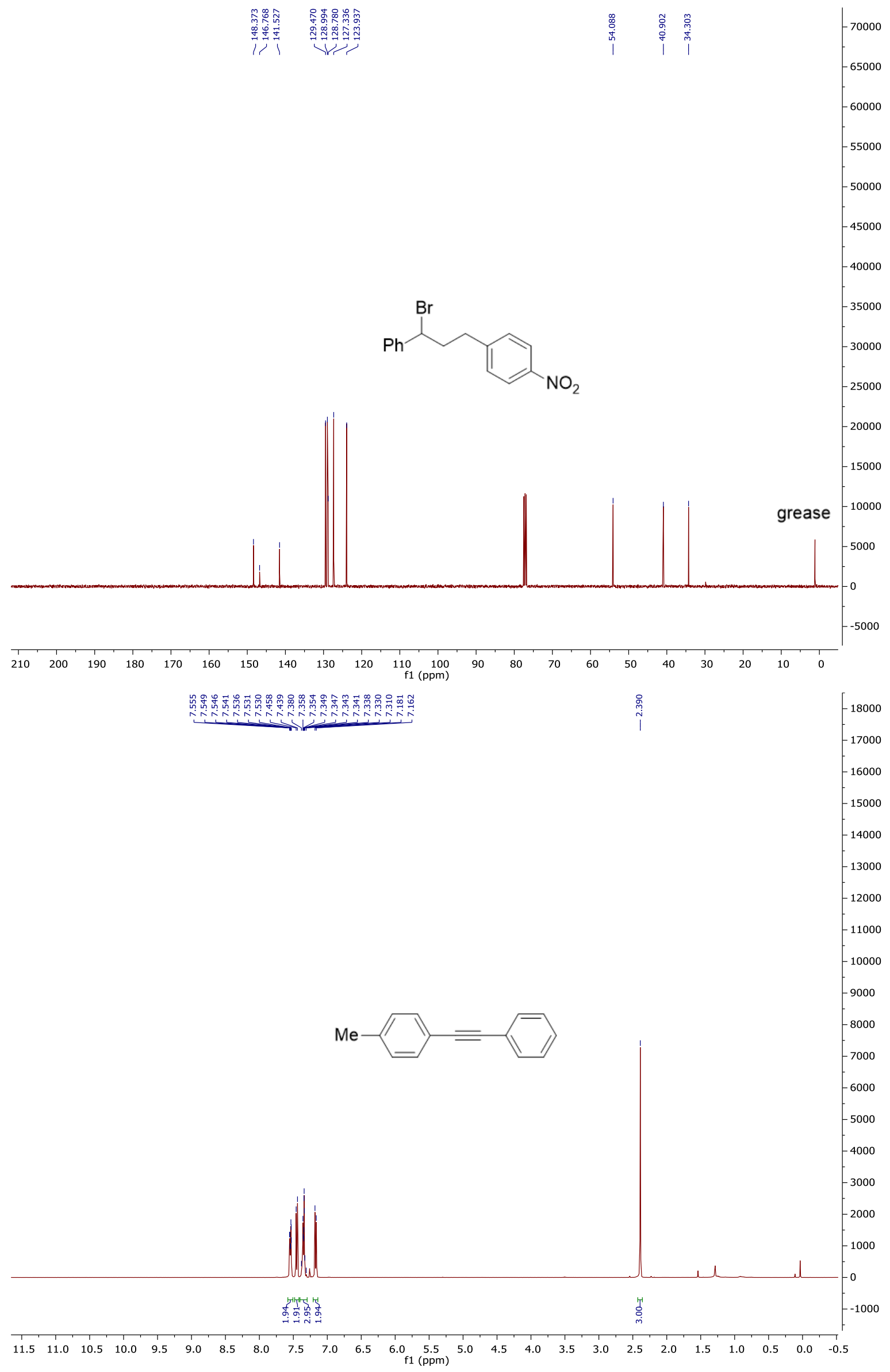




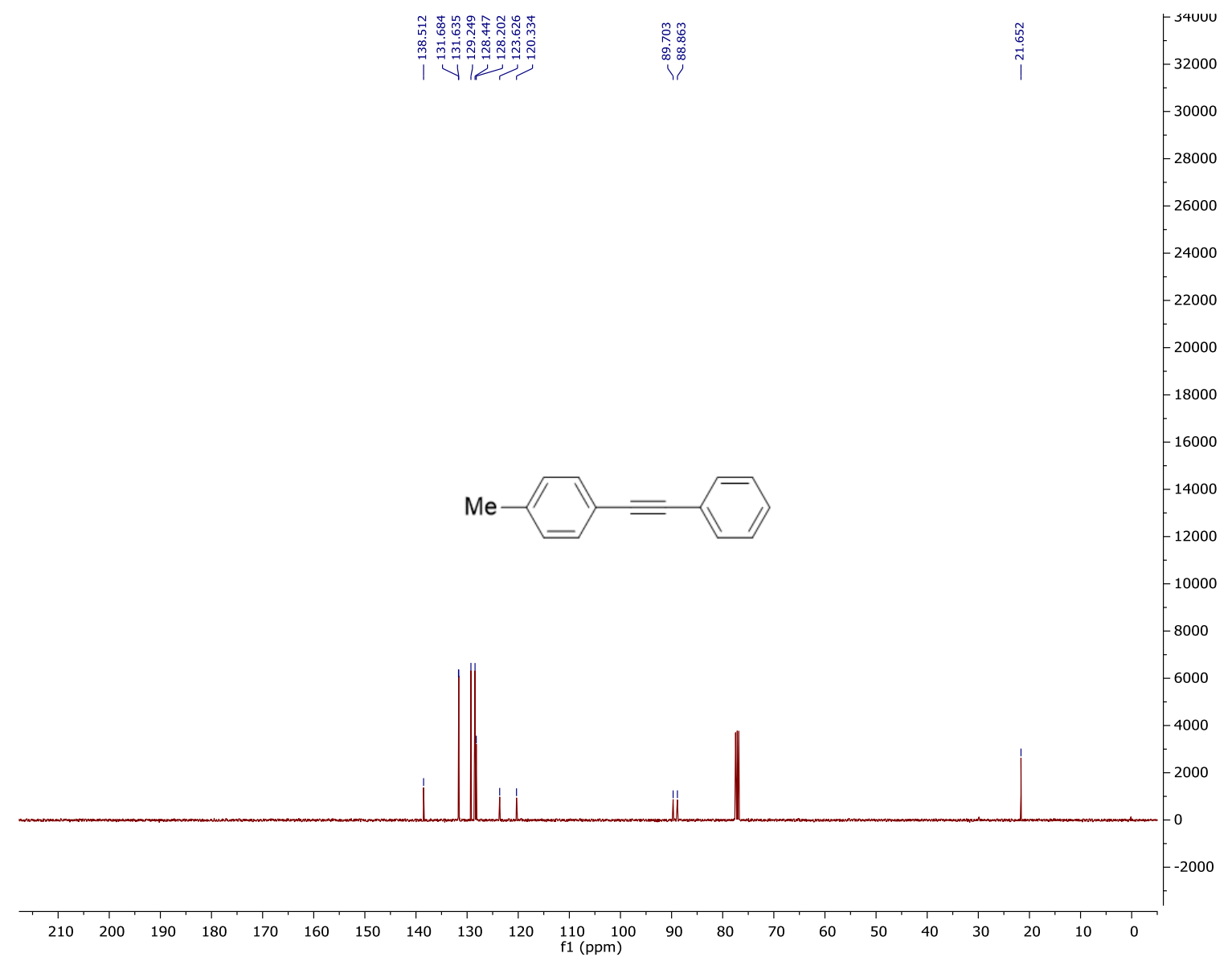

18. References

(1) Yang, Q. L.; Li, C. Z.; Zhang, L. W.; Li, Y. Y.; Tong, X.; Wu, X. Y.; Mei, T. S. PalladiumCatalyzed Electrochemical C-H Alkylation of Arenes. Organometallics 2019, 38, 1208-1212.

(2) Sharma, P.; Rohilla, S.; Jain, N. Copper Acetate-DMSO Promoted Methylthiolation of Arenes and Heteroarenes. J. Org. Chem. 2015, 80, 4116-4122.

(3) Neufeldt, S. R.; Sanford, M. S. O-Acetyl Oximes as Transformable Directing Groups for PdCatalyzed C-H Bond Functionalization. Org. Lett. 2010, 12, 532-535.

(4) Hari, D. P.; Schroll, P.; König, B. Metal-Free, Visible-Light-Mediated Direct C-H Arylation of Heteroarenes with Aryl Diazonium Salts. J. Am. Chem. Soc. 2012, 134, 2958-2961.

(5) Li, Z.; Li, C. J. CuBr-Catalyzed Direct Indolation of Tetrahydroisoquinolines via CrossDehydrogenative Coupling between sp3 C-H and sp2 C-H Bonds. J. Am. Chem. Soc. 2005, 127, 6968-6969.

(6) An, J.; Lombardi, L.; Grilli, S.; Bandini, M. PPh3AuTFA Catalyzed in the Dearomatization of 2-Naphthols with Allenamides. Org. Lett. 2018, 20, 7380-7383.

(7) Laursen, B. W.; Krebs, F. C. Synthesis of a Triazatriangulenium Salt. Angew. Chem., Int. Ed. 2000, 39, 3432-3434.

(8) Delgado, I. H.; Pascal, S.; Wallabregue, A.; Duwald, R.; Besnard, C.; Guénée, L.; Nançoz, C.; Vauthey, E.; Tovar, R. C.; Lunkley, J. L.; Muller, G.; Lacour, J. Functionalized Cationic [4]Helicenes with Unique Tuning of Absorption, Fluorescence and Chiroptical Properties up to the Far-Red Range. Chem. Sci. 2016, 7, 4685-4693.

(9) Sørensen, T. J.; Nielsen, M. F.; Laursen, B. W. Synthesis and Stability of N,N'-Dialkyl-1,13- 
Dimethoxyquinacridinium (DMQA+): A [4]Helicene with Multiple Redox States. ChemPlusChem 2014, 79, 1030-1035.

(10) Romero, N. A.; Nicewicz, D. A. Organic Photoredox Catalysis. Chem. Rev. 2016, 116, 1007510166.

(11) Zuo, Z.; Cong, H.; Li, W.; Choi, J.; Fu, G. C.; MacMillan, D. W. C. Enantioselective Decarboxylative Arylation of $\alpha$-Amino Acids via the Merger of Photoredox and Nickel Catalysis. J. Am. Chem. Soc. 2016, 138, 1832-1835.

(12) Kalyani, D.; McMurtrey, K. B.; Neufeldt, S. R.; Sanford, M. S. Room-Temperature C-H Arylation: Merger of Pd-Catalyzed C-H Functionalization and Visible-Light Photocatalysis. J. Am. Chem. Soc. 2011, 133, 18566-18569.

(13) Yu, S.; Liu, Z.; Zhang, J.; Li, H.; Xu, N.; Yuan, X. X.; Wu, Y. An Azo-Coupling ReactionBased Surface Enhanced Resonance Raman Scattering Approach for Ultrasensitive Detection of Salbutamol. RSC Adv. 2018, 8, 5536-5541.

(14) Zou, Y. Q.; Chen, J. R.; Liu, X. P.; Lu, L. Q.; Davis, R. L.; Jørgensen, K. A.; Xiao, W. J. Highly Efficient Aerobic Oxidative Hydroxylation of Arylboronic Acids: Photoredox Catalysis Using Visible Light. Angew. Chem., Int. Ed. 2012, 51, 784-788.

(15) Pitre, S. P.; McTiernan, C. D.; Ismaili, H.; Scaiano, J. C. Mechanistic Insights and Kinetic Analysis for the Oxidative Hydroxylation of Arylboronic Acids by Visible Light Photoredox Catalysis: A Metal-Free Alternative. J. Am. Chem. Soc. 2013, 135, 13286-13289.

(16) Yu, X.; Cohen, S. M. Photocatalytic Metal-Organic Frameworks for the Aerobic Oxidation of Arylboronic Acids. Chem. Commun. 2015, 51, 9880-9883.

(17) Xie, H. Y.; Han, L. S.; Huang, S.; Lei, X.; Cheng, Y.; Zhao, W.; Sun, H.; Wen, X.; Xu, Q. L. N-Substituted 3(10H)-Acridones as Visible-Light, Water-Soluble Photocatalysts: Aerobic Oxidative Hydroxylation of Arylboronic Acids. J. Org. Chem. 2017, 82, 5236-5241.

(18) Nguyen, J. D.; Tucker, J. W.; Konieczynska, M. D.; Stephenson, C. R. J. Intermolecular Atom Transfer Radical Addition to Olefins Mediated by Oxidative Quenching of Photoredox Catalysts. J. Am. Chem. Soc. 2011, 133, 4160-4163.

(19) Wallentin, C. J.; Nguyen, J. D.; Finkbeiner, P.; Stephenson, C. R. J. Visible Light-Mediated Atom Transfer Radical Addition via Oxidative and Reductive Quenching of Photocatalysts. J. Am. Chem. Soc. 2012, 134, 8875-8884.

(20) Pirtsch, M.; Paria, S.; Matsuno, T.; Isobe, H.; Reiser, O. [Cu(Dap) $2 \mathrm{Cl}]$ as an Efficient VisibleLight-Driven Photoredox Catalyst in Carbon-Carbon Bond-Forming Reactions. Chem. - Eur. J. 2012, 18, 7336-7340.

(21) Paria, S.; Pirtsch, M.; Kais, V.; Reiser, O. Visible-Light-Induced Intermolecular AtomTransfer Radical Addition of Benzyl Halides to Olefins: Facile Synthesis of Tetrahydroquinolines. Synthethis 2013, 45, 2689-2698.

(22) Rawner, T.; Lutsker, E.; Kaiser, C. A.; Reiser, O. The Different Faces of Photoredox Catalysts: Visible-Light-Mediated Atom Transfer Radical Addition (ATRA) Reactions of Perfluoroalkyl Iodides with Styrenes and Phenylacetylenes. ACS Catal. 2018, 8, 3950-3956.

(23) Pavlishchuk, V. V; Addison, A. W. Conversion Constants for Redox Potentials Measured versus Different Reference Electrodes in Acetonitrile Solutions at $25{ }^{\circ} \mathrm{C}$. Inorg. Chim. Acta 2000, 298, 97-102.

(24) Mohammad, M.; Kosower, E. M. Stable Free Radicals. VI. Reaction between 1-Ethyl-4Carbomethoxypyridinyl Radical and 4-Nitrobenzyl Halides. J. Am. Chem. Soc. 1971, 93, 2713-2719. 
(25) Wayner, D. D. M.; McPhee, D. J.; Griller, D. Oxidation and Reduction Potentials of Transient Free Radicals. J. Am. Chem. Soc. 1988, 110, 132-137. 